\title{
Interne Strukturen und Prozesse öffentlicher Organisationen
}

\section{Lernziele}

am Ende dieses Kapitels sollten Sie

- einen Überblick über die zentralen Elemente, Vorteile und Probleme bürokratischer Organisationen und Verfahren haben;

- deren Ausgestaltung auf der Ebene der Ministerien und Kommunen in der Bundesrepublik sowie die wichtigsten Kritikpunkte kennen;

- unterschiedliche Möglichkeiten von Planung und Entscheidung in der öffentlichen Verwaltung und wichtige klassische Entscheidungstheorien kennen;

- die vielfältigen Beziehungen zwischen Politik und Verwaltung und die dabei relevanten normativen Vorstellungen verstehen; sowie

- erkennen, dass es in der Politikberatung meistens um Verwaltungsberatung geht.

Während die bisherige Darstellung die öffentliche Verwaltung vorrangig aus der Makro-Perspektive betrachtet hat, sich also vor allem auf Aufgaben und interorganisatorische Beziehungen öffentlicher Organisationen auf verschiedenen Ebenen und in verschiedenen Sektoren bezogen hat, geht es im Folgenden um das „Innenleben“ solcher Organisationen. Im Mittelpunkt steht die Mikro-Perspektive, also intraorganisatorische Strukturen und Prozesse. Dies ist auch eine klassische Perspektive der Organisations- und Verwaltungssoziologie (vgl. Mayntz 1997, Derlien 1984).

Zunächst ist festzuhalten, dass die öffentliche Verwaltung nicht nur vielfältige Aufgaben und Funktionen wahrnimmt, sondern auch aus einer großen Vielfalt unterschiedlicher öffentlicher Organisationen besteht. Neben den bekannten und besonders sichtbaren Ministerien auf Bundes- und Landesebene und den Kommunalverwaltungen gibt es eine Fülle von 
- nachgeordneten Behörden (von der Polizei über den Denkmalschutz oder die Gesundheitsämter bis hin zu Forschungsanstalten und Forstämtern),

- Oberbehörden (vom Bundesamt für Familie und zivilgesellschaftliche Aufgaben bis zur Bundesnetzagentur),

- Anstalten (von der Bundesanstalt für Finanzdienstleistungsaufsicht bis hin zur Bundesanstalt für Vereinigungsbedingte Sonderaufgaben),

- Körperschaften (von der Bundesversicherungsanstalt bis hin zur Bundesagentur für Arbeit) und

- öffentlich-rechtlichen Stiftungen (von der Stiftung Mutter und Kind bis hin zur Stiftung Preußischer Kulturbesitz).

Eine Universität ist somit genauso eine öffentliche Organisation wie eine Strafvollzugsanstalt, eine Schule oder ein Museum, und damit Teil der öffentlichen Verwaltung. Im allgemeinen Sprachgebrauch spricht man oft von Behörden, und auch im Verwaltungsverfahrensgesetz heißt es lapidar „Behörde im Sinne dieses Gesetzes ist jede Stelle, die Aufgaben der öffentlichen Verwaltung wahrnimmt“ ( $\$ 1$ Abs. 4 VwVfG). Die meisten Schulen und Universitäten würden sich allerdings heute wohl dagegen verwahren, als „Behörde“ bezeichnet zu werden, und auch die alte Bundesanstalt für Arbeit wollte eine Bundesagentur werden, um damit zumindest nach außen den Wandel von einer Behörde zum modernen Dienstleister zu vollziehen. Auch wenn daher der Begriff „öffentliche Verwaltung“ vielschichtig und missverständlich ist, gibt es dennoch ein Merkmal, das zumindest in der alltäglichen Wahrnehmung mit fast allen öffentlichen Organisationen verbunden wird und als charakteristisch für deren interne Strukturen und Prozesse gilt, das der Bürokratie (siehe zum Folgenden als systematische Einführung Derlien/Böhme/Heindl 2011).

\subsection{Bürokratie und Bürokratiekritik}

\subsubsection{Merkmale bürokratischer Organisation}

Der Begriff der Bürokratie ist zunächst als Schimpfwort erfunden worden (vgl. Albrow 1972). Er geht auf den Franzosen de Gournay zurück, der - lange vor der französischen Revolution - damit die Herrschaft des „Büros“, die nicht-legitimierte Herrschaft von Subalternen kritisiert hat. Er definierte damit Bürokratie ganz explizit als vierte Herrschaftsform neben Monarchie, Aristokratie und Demokratie, als Regierungsform, in der Regieren und Verwalten zum Selbstzweck geworden sei. Erst viel später, zu Beginn des 20. Jahrhunderts, wurde dieser Begriff und das 
damit zusammenhängende Konzept von Max Weber wissenschaftlich „neutralisiert" und objektiviert.

Max Weber, geboren 1864 in Erfurt, studierte Jura, Geschichte, Nationalökonomie und Philosophie in Heidelberg, Göttingen und Berlin. 1886 promovierte er an der juristischen Fakultät in Berlin mit einer Arbeit „Zur Geschichte der Handelsgesellschaften im Mittelalter“, drei Jahre später folgte die Habilitation über die „Die römische Agrargeschichte in ihrer Bedeutung für das Staats- und Privatrecht", ebenfalls in Berlin. 1893 wird er auf die Professur für Nationalökonomie in Freiburg berufen, 1897 wechselt er nach Heidelberg, wo er bis 1903 ordentlicher Professor bleibt. Nach einer längeren, zunächst krankheitsbedingten Pause nahm er erst 1919 mit einem Lehrstuhl für Gesellschaftswissenschaft, Wirtschaftsgeschichte und Nationalökonomie in München die Lehrtätigkeit wieder auf, stirbt aber bereits 1920 an einer Lungenentzündung. Neben seiner wissenschaftlichen Arbeit engagierte sich Weber für sozialpolitische Fragen, unter anderem im „Verein für Socialpolitik“.

Das Werk Max Webers ist äußerst vielfältig und umfangreich. Neben eher historischen Werken umfasst es vor allem Werke zur Philosophie und Methode der Sozialwissenschaften, religionssoziologische Werke über die Rolle der protestantischen Ethik bei der Entwicklung des modernen Kapitalismus und schließlich sein posthum veröffentlichtes Hauptwerk „Wirtschaft und Gesellschaft“, in dem er die Grundzüge einer verstehenden Soziologie entwickelt. Von besonderer Bedeutung für die Politik- und Verwaltungswissenschaft sind Max Webers Überlegungen zur Bürokratie. In „Wirtschaft und Gesellschaft“ konzipiert Max Weber den Idealtypus der bürokratischen (legalen) Herrschaft, die er mit den Formen der traditionellen (patriarchalischen) und der charismatischen Herrschaft kontrastiert. Für Max Weber war die Herausbildung einer Bürokratie Ausdruck der Rationalisierung der Institutionen in einer zunehmend als berechenbar und beherrschbar empfundenen natürlichen Welt. Rund 100 Jahre später prägt Max Webers Analyse nach wie vor viele Vorstellungen über Politik und Verwaltung. Mit seinem umfassenden Werk ist Max Weber einer der Begründer der Soziologie und der modernen Sozialwissenschaften, mit seiner Analyse zur Bürokratie ist er zudem Wegbereiter der modernen Organisationstheorie und der Verwaltungswissenschaften.

In einem berühmten Zitat postuliert Max Weber:

„Die rein bureaukratische, also: bureaukratisch-monokratische aktenmäßige Verwaltung ist nach allen Erfahrungen die an Präzision, Stetigkeit, Disziplin, Straffheit und Verlässlichkeit, also: Berechenbarkeit für den Herrn wie für die Interessenten, Intensität und Extensität der Leistung, formal universeller Anwendbarkeit auf alle Aufgaben, in all diesen Bedeutungen: formal rationalste Form der Herrschaftsausübung" (Weber 1921). 
Als Merkmale einer solchen bürokratischen Organisation hebt er besonders hervor: ${ }^{59}$

- Hauptamtliches Personal (Trennung von Amt und Person und von öffentlichen und privaten Mitteln);

- Fachlichkeit, Professionalisierung (Einstellung und Beförderung nach Ausbildung und Leistung);

- Arbeitsteilung und Spezialisierung;

- Hierarchische Über- und Unterordnung (klare Zuständigkeiten, Dienstweg);

- Regelgebundenheit sowie

- Schriftlichkeit, Aktenmäßigkeit.

Bei der Interpretation dieser Merkmale ist ihr historischer Kontext zu betonen (vgl. Derlien 1989). Für Max Weber ist bürokratische Organisation eine wichtige Errungenschaft und eine rationale Form der Herrschaft, weil sie überkommene feudale, willkürliche Herrschaftsformen ersetzt, also etwa die Aneignung öffentlicher Mittel durch die Besitzer von Ämtern, die Ausübung dieser Ämter und die Einstellung und Beförderung auf der Grundlage von Vererbung, Nepotismus oder Zugehörigkeit zu einer bestimmten Gruppe, unklare, kollegiale Zuständigkeiten und Verantwortlichkeiten, unprofessionelle Verwaltung durch Amateure und Begünstigte und insbesondere undurchschaubare und unkontrollierte Willkür. Fachlichkeit, Unpersönlichkeit, Berechenbarkeit sind daher zentrale Merkmale des weberschen Bürokratiebegriffs. Dass dies auch heute noch wichtige Errungenschaften sind, kann man sich schnell verdeutlichen, wenn man sich z. B. klar macht, was eine in diesem Sinne unbürokratische amtliche Entscheidung wäre, also etwa „so wie Sie aussehen, bekommen Sie hier gar nichts“ (schnell, persönlich und nicht durch Paragraphenreiterei behindert).

Die Webersche Bürokratietheorie gehört sicherlich zu den wichtigsten Grundlagen der Verwaltungswissenschaft, und sie ist auch im gesamten Kanon der Sozialwissenschaften eine der bekanntesten und einflussreichsten Theorien. Allerdings ist sie auch immer wieder selektiv oder falsch interpretiert worden, etwa als allgemeingültige empirische Beschreibung vorhandener Organisationsformen oder als normative und präskriptive Vorschrift, als Modell, wie öffentliche und formale Organisationen gestaltet werden sollten. Beides trifft für Weber gerade nicht zu, der bestimmte in der Wirklichkeit vorfindbare Merkmale von Organisationen typologisch zusammenfasst (und durchaus auch kritisch sieht). Er nennt dies zwar

59 Da Weber Bürokratie an unterschiedlichen Stellen definiert, gibt es keine definitive Enumeration, unterschieden werden $u$. a. auch die Personal- und Organisationsmerkale von Bürokratien (vgl. im Detail Derlien u.a. 2011). 
einen „Idealtypus“, aber damit ist kein normatives Ideal gemeint, sondern eine analytische Beschreibung, die in dieser reinen Form historisch nicht vorkommt. Eine mögliche und sinnvolle Frage ist aber, ob denn vorhandene Organisationen Merkmale bürokratischer Organisationen aufweisen.

Tatsächlich weisen öffentliche Organisationen in Deutschland, insbesondere im Kernbereich von Ministerial- und Kommunalverwaltung die klassischen bürokratischen Merkmale in hohem Maße auf. Die von Max Weber besonders hervorgehobenen Merkmale der professionellen Verwaltung, also Hauptamtlichkeit, Einstellung und Beförderung nach Ausbildung, Leistung und Seniorität sind bereits im Abschnitt 3.6 „Personal“ ausführlich behandelt worden und finden sich besonders ausgeprägt im deutschen Beamtenrecht. Beamte werden aufgrund ihrer i.d.R. durch einen Abschluss nachgewiesenen Qualifikation für eine bestimmte Laufbahn eingestellt (etwa allgemeiner Verwaltungsdienst, Forstdienst, Schuldienst, Bauverwaltung etc.), stehen zum Dienstherrn in einem besonderen Vertragsverhältnis, werden geldlich entlohnt (alimentiert) und die Arbeitsmittel sind kein persönlicher Besitz. Wie Hans-Ulrich Derlien bemerkt, besitzt heute kein Wissenschaftler die teuren Laboreinrichtungen, mit denen er arbeitet, und während der Kavallerist sich früher in der Regel selbst ausrüsten musste, ,ist es für uns ganz undenkbar, dass sein historischer Nachfolger, der Panzeraufklärer, noch im Besitz dieses Verwaltungsmittels ist“" (Derlien 1989, S. 323).

Die übrigen oben aufgeführten Merkmale bürokratischer Organisationen können unter den gängigen Begriffen „Aufbauorganisation“, also formale Gliederung durch Arbeitsteilung und Hierarchie, und „Ablauforganisation“, also Regelgebundenheit, Aktenmäßigkeit und Schriftlichkeit zusammengefasst werden, wobei beide Organisationsmerkmale voneinander abhängen.

\subsubsection{Aufbauorganisation: Spezialisierung und Hierarchie}

Die deutsche Verwaltung ist durch eine starke Arbeitsteilung, Spezialisierung und Differenzierung gekennzeichnet - auf die Vielfalt der deutschen Behördenlandschaft ist bereits verschiedentlich verwiesen worden. Schematisch kann man unterschiedliche Typen von Spezialisierung und Differenzierung danach unterscheiden, ob sie zwischen (interorganisatorisch) oder innerhalb von Organisationen (intraorganisatorisch) stattfindet, und ob es sich um horizontale oder vertikale Differenzierung handelt. 


\begin{tabular}{lll}
\hline & interorganisatorisch & intraorganisatorisch \\
\hline horizontal & - Ressorts, Ministerien & - Abteilungen \\
& - Dezernate & - Referate \\
& - Sonderbehörden & - Sachgebiete \\
\hline vertikal & - Dezentralisierung, & - Hierarchieebenen \\
& - Föderalismus & \\
& - nachgeordnete Behörden & \\
& (Dekonzentration) & \\
& - Auslagerungen, & \\
& Outsourcing & \\
\hline
\end{tabular}

Abb. 43 Formen von Spezialisierung und Differenzierung

Quelle: eigene Darstellung

So wird zunehmende Arbeitsteilung und Spezialisierung auf der horizontalen Ebene in der Anzahl von Fachressorts, Ministerien oder Dezernaten und innerhalb dieser durch die Anzahl von Abteilungen, Referaten oder Sachgebieten deutlich. Vertikal gibt es Dezentralisierung etwa im Rahmen kommunaler Selbstverwaltung, aber auch durch die Zunahme nachgeordneter Fachbehörden und die verschiedenen Formen der Auslagerung etwa in Landesbetriebe, über GmbHs bis hin zur reinen Privatisierung. Innerhalb von Organisationen ist die Erhöhung der Anzahl von Hierarchieebenen zu nennen, also etwa Hauptabteilungen, Abteilungen, Referatsgruppen, Referate, Sachgebiete etc. Der Grund für diese zunehmende Arbeitsteilung liegt, einmal abgesehen von internen Gründen (etwa dem Eigeninteresse der Beschäftigten, s. u.), in der mit dem Begriff "requisite variety“ benannten Beobachtung, dass die Fähigkeit einer Organisation auf eine komplexe Umwelt zu reagieren, eine entsprechende Eigenkomplexität erfordert. Eine Verwaltung in einer kleinen Gemeinde wird so mit einigen wenigen Abteilungen auskommen, eine Großstadtverwaltung kommt nicht umhin, nicht nur das Sozialamt vom Jugendamt zu trennen, sondern auch innerhalb des Sozialamts oder z. B. des Bauamts verschiedene Abteilungen und Sachgebiete auszuweisen (Hochbau, Tiefbau etc.).

Der große Vorteil dieser Spezialisierung ist die Fachkompetenz. Kindergärtnerinnen sind nicht für Bauanträge zuständig und Polizisten nicht für die Gesundheitsvorsorge - dafür gibt es speziell ausgebildete Mitarbeiter und Facheinheiten. Eine gesicherte, feste Zuständigkeit ist ein großer Vorteil sowohl für die Kunden und Klienten der Verwaltung als auch für die politisch Verantwortlichen.

Zur Beschreibung und Analyse der horizontalen Differenzierung von Organisationen oder von organisatorischen Feldern unterscheidet man seit dem grundlegenden Werk von Gulick/Urwick (1937) folgende Organisationsprinzipien: 
- nach Objekt (divisional): alle Personen, die für dieselbe öffentliche Aufgabe arbeiten, werden in einer Einheit zusammengefasst (Beispiel: Umwelt, Denkmalschutz);

- nach Verrichtung (funktional): alle Personen einer Berufsgruppe bzw. alle, die mit denselben Kenntnissen und Techniken arbeiten (Planungsabteilung, Haushalt, Bibliothek, Forschung, Labore);

- nach Klientel (klientelistisch): alle die mit denselben Personen- oder Sachgruppen arbeiten (Frauenministerium, Jugendamt);

- nach Bezirk (regional): alle in einem abgegrenzten Bezirk (Bezirksamt, Quartiersmanagement, Bezirksregierung).

Es gibt keine „beste“ Lösung der Organisation von Aufgaben nach diesen Prinzipien, zumal die Verbindungen zwischen ihnen offenkundig sind, sondern jedes dieser Organisationsprinzipien hat Vor- und Nachteile. In der Realität findet man eine Kombination der verschiedenen Prinzipien, die naturgemäß zu Koordinationsproblemen führt und immer wieder hinterfragt wird und werden muss.

Im Rahmen der vertikalen Differenzierung ist insbesondere das Prinzip der hierarchischen Linienorganisation für bürokratische Organisationen von entscheidender Bedeutung. Im klassischen Einliniensystem ist jede Organisationseinheit genau einer anderen unterstellt. In dieser klassischen Autoritätshierarchie gibt es also feste, eindeutige Strukturen für Kommunikation, Weisungen und Kontrolle. Verantwortlichkeiten sind klar verteilt, und damit auch Zuständigkeiten. Wer BAföG braucht, bekommt es nicht im Bauamt, aber auch nicht in einem Büro, das für einen anderen Stadtteil oder Buchstaben zuständig ist. Hier liegt einer der Gründe für die klassische Bürokratiekritik: Man muss erst die für sein Anliegen „zuständige" Einheit finden, und das ist manchmal nicht ganz einfach.

Für die vertikale Differenzierung und Organisation sind zwei weitere Prinzipien von großer Bedeutung, nämlich Leitungsspanne und Leitungstiefe. Die Leitungsspanne, auch Kontrollspanne genannt (engl. span of control) gibt an, wie viele Einheiten oder Personen einer übergeordneten Einheit (oder einem Vorgesetzten) unmittelbar, also auf der direkt folgenden Leitungsebene, unterstellt sind. Die Leitungstiefe gibt dann die Anzahl der Leitungs- oder Organisationsebenen an, aus denen eine Organisation besteht. Offensichtlich hängen beide Konzepte eng zusammen: je größer die Leitungs- oder Kontrollspanne, desto geringer die Leitungstiefe, also desto flacher die Organisation (bei einem festen Bestand von Personal). Auch hier gibt es keine einfachen „optimalen“ Bezugsgrößen, keinen einfachen „best way“ der Organisation, obwohl es seit längerem generell eine Präferenz für eine möglichst geringe Leitungstiefe gibt (Minimalebenenprinzip, also "flache Organisationen“) (vgl. ausführlich Reichard 1987, S. 184ff.). Der Sinn einer starken vertikalen Diffe- 
renzierung, also vieler Hierarchieebenen, liegt selbstverständlich in dem Ziel der möglichst direkten Weisung und Kontrolle: je kleiner die Leitungsspanne, desto besser können untergeordnete Einheiten überwacht werden.

Eine differenzierte Hierarchie über- und untergeordneter Einheiten mit eindeutigem Dienstweg (Einliniensystem) sowie eine klare Kompetenzverteilung mit starker Entscheidungszentralisation (und als Folge erheblicher Leitungstiefe) sind klassische Merkmale von Bürokratien. Die damit verbundenen Aufgabenzuweisungen sind wiederum in Form von Geschäftsverteilungsplänen schriftlich fixiert, die die innerbehördliche Arbeitsteilung und vor allem eindeutige Zuständigkeiten festschreiben. Im Prinzip kann so jede Aufgabe, jeder „Vorgang“, jedes Schreiben, das eine Behörde erreicht, aufgrund des Geschäftsverteilungsplans einer eindeutig zuständigen Einheit zugewiesen werden.

\subsubsection{Ablauforganisation: Aktenmäßigkeit und Geschäftsordnung}

Aber nicht nur die Aufbauorganisation ist in einer Bürokratie schriftlich fixiert, sondern auch die Ablauforganisation, also die Prozesse, nach denen innerhalb der Organisation gearbeitet werden soll. Eine Bürokratie zeichnet sich aus durch ein System von genau definierten Verfahrensweisen für die Erfüllung von Aufgaben. Bürokratisches und damit Verwaltungshandeln ist also stark regelgebunden und standardisiert. Die Verwaltung handelt, im Prinzip, nur aufgrund schriftlich fixierter (und damit transparenter, überprüfbarer) Regeln, und sie dokumentiert ihre Aktivitäten schriftlich (Aktenmäßigkeit).

Beide Prinzipien, Regelgebundenheit und Aktenmäßigkeit, haben erhebliche Vorteile. Zusammen mit Hierarchie und Spezialisierung verhindern sie, so zumindest die theoretische Annahme, Willkür und Inkompetenz und ermöglichen erst rechtsstaatliche und demokratische Führung und Kontrolle der Verwaltung. Sie sind die Garanten von Fachlichkeit, Berechenbarkeit und Unpersönlichkeit, d.h. der Behandlung „ohne Ansehen der Person“, „sine ira et studio“. Aus diesem Grund regeln Geschäftsordnungen oft bis ins kleinste Detail, wie Prozesse innerhalb der Bürokratie ablaufen sollen, also von der Behandlung der Eingänge (Wer sieht welche Eingänge? Wer entscheidet über die Verteilung? Wie werden Aktenzeichen vergeben?), die Bearbeitung von Vorgängen (Wer ist zeichnungsberechtigt? Wie müssen andere Einheiten beteiligt werden? Welche Form soll ein Vermerk haben?) bis hin zu Fragen bei der Abwicklung „besonderer Dienstgeschäfte“ (Kontakte mit Klienten oder Interessengruppen, Dienstreisen und Dienstgänge) und der Dienstoder Hausordnung.

So wird in klassischen Geschäftsordnungen u. a. geregelt, wer mit welcher Farbe Vermerke abzeichnen darf (Leitungsebene mit grün, Staatssekretäre mit rot, Abteilungsleiter mit violett etc.), welche Zeichen auf einem Schreiben was bedeuten 
sollen, welche Vorgänge „vor Abgang z.Kts.“ gegeben werden müssen usw. Der Sinn dieser Vorschriften ist, dass im Nachhinein auf jeder Akte erkennbar sein muss, wer sie gesehen und daher „abgezeichnet" hat bzw. wer welche „Verfügungen“ auf die Akte geschrieben hat - alles im Interesse der Berechenbarkeit und Nachprüfbarkeit. In einer klassischen Bürokratie müssen Probleme a.d. D., „auf dem Dienstweg“, gelöst werden, d.h. Eingänge werden in der untersten Ebene bearbeitet und laufen dann die Hierarchieleiter hoch, bis sie vom jeweils zuständigen „Letztentscheider“, ggf. dem Minister oder Bürgermeister, entschieden und unterzeichnet werden. Der Dienstweg ist die getreue Abbildung der innerbehördlichen Verantwortung. Offensichtlich werden diese klassischen Kommunikationswege in modernen Verwaltungen durch elektronische Kommunikation (Emails, elektronische Akten etc.) überlagert, die die gleichzeitige Beteiligung vieler Stellen prinzipiell sehr einfach machen würde. Insbesondere mit Systemen „elektronischer Aktenführung“ wird dabei versucht, die klassische Nachvollziehbarkeit von Entscheidungen auch in der Welt der digitalen Kommunikation abzubilden.

Bei umfangreicher Spezialisierung, Hierarchisierung, Aktenmäßigkeit und Regelgebundenheit ist die gegenseitige Kommunikation, Integration und Koordination der unterschiedlichen Einheiten ein besonderes Problem, denn es kann keineswegs davon ausgegangen werden, dass alle Einheiten einer Organisation, also etwa das Umwelt- und das Verkehrsamt einer Kommune die gleichen Interessen verfolgen und nach den gleichen Rationalitätskriterien entscheiden, vom Jugendamt ganz zu schweigen. Eine bürokratische Organisation ist also keineswegs konfliktfrei, ganz im Gegenteil. Größere Spezialisierung bedeutet gleichzeitig fachliche Vertiefung und Verengung, sog. „selektive Problemperzeption" oder auch „tunnel view“ und im Extrem Fachidiotentum. Straßenbauämter sind dafür da, dass sie sich mit Straßenbau beschäftigen, für Landschaftsschutz, Denkmalpflege, Gewässerschutz und Wirtschaftsförderung sind andere Einheiten zuständig. Inner- und interorganisatorische Konflikte sind daher keine zu überwindende Schwäche, kein Mangel von Verwaltungen, sondern sind gewollt und notwendig. Sie sind, wenn man so will, der eigentliche Sinn von Organisationen. Spezialisierung und Koordination sind zwei Seiten der gleichen Münze, je spezialisierter die Verwaltung, desto mehr Konflikte und desto mehr Bedarf an Koordination. Ein zentrales Problem für bürokratische Organisationen ist daher die Koordination unterschiedlicher Interessen und die Lösung der zwischen ihnen auftretenden Konflikte.

\subsubsection{Koordination}

Jeder kennt Koordinationsprobleme öffentlicher Organisationen. Kaum ist die Straße nach langwierigen Bauarbeiten fertig, wird sie wieder aufgerissen, weil noch irgendwelche Kabel verlegt werden müssen. Während die Agrarverwaltung die intensive Viehzucht durch Sonderprogramme fördert, versucht die Umweltverwaltung die 
Verseuchung der Bäche durch Gülle zurückzudrängen, die Verkehrsverwaltung plant eine neue Autobahn und eine Umgehungsstraße, und der Naturschutz will ganze Areale jeglicher Nutzung entziehen.

In einer zunehmend vernetzten und interdependenten Gesellschaft nehmen diese Koordinationsprobleme offenkundig zu. Agrarpolitik hängt eng mit Naturschutz, Verbraucherschutz oder auch Regionalpolitik zusammen, die Bewältigung von Migration erfordert die Zusammenarbeit u. a. der Sozial-, Schul-, Ausländer- und Jugendämter und Jobcentern, von neuartigen Problemen wie Klimapolitik oder nachhaltige Entwicklung ganz zu schweigen. Wissenschaftlich werden diese Probleme seit einiger Zeit unter der Überschrift wicked problems (vertrackte Probleme) diskutiert (als Übersicht Fuhr 2019 und das Schwerpunktheft von dms 1/2016). Sie zeichnen sich ganz allgemein durch besondere Komplexität, Unsicherheit und Ambiguität aus:

- sie sind komplex, weil sie viele gesellschaftliche Akteure, Sektoren und Ebenen (und damit viele unterschiedliche Verwaltungen) und auch unterschiedliche Zeithorizonte betreffen (multi-actor, -sector, -level and -temporal);

- sie sind unsicher, weil Wirkungsketten und Interdependenzen lang und wenig oder gar nicht bekannt, Effekte daher kaum vorhersehbar und Wissensbestände kontrovers sind (contested knowledge);

- und sie sind mehrdeutig (ambiguos), weil sie widersprüchliche Werte, Weltanschauungen und Bewertungskriterien umfassen und daher keine gemeinsamen normativen Standards vorhanden sind.

Die Koordinationsprobleme moderner Gesellschaften und politisch-administrativer Systeme werden international seit Jahren diskutiert, Schlagworte sind joined-up government oder whole of government (Pollitt 2003, Christensen/Laegreid 2007, Kuhlmann/Bogumil 2019b). Grundlegende Konzepte, wie Koordination durch Weisung (Hierarchie), Verhandlung (Netzwerke), Solidarität (Gemeinschaften) oder Tausch (Märkte) sind bekannt, aber es gibt keine übergreifende und allgemeine Theorie der Koordination (grundlegend immer noch Wildavsky 1973, als Überblick Alexander 2014, Hustedt/Veit 2014 und die Beiträge im Schwerpunktheft von dms 1/2014).

Ein besonderer Koordinierungsbedarf entsteht zudem durch die starke Verwaltungsverflechtung, die strukturell im deutschen Verwaltungsföderalismus verankert ist (vgl. Kap. 3.2.3). Dann wenn Zuständigkeiten zu stark zwischen verschiedenen Ebenen verteilt sind, können Doppelarbeiten, Koordinierungsprobleme und eine fehlende Verantwortung für den Gesamtprozess auftreten. Insbesondere der Bereich der Migrations- und Integrationspolitik ist in jüngster Zeit als Beispiel für eine sol- 
che besondere Verwaltungsverflechtung identifiziert worden. In diesem Politikfeld werden die Aufgaben vertikal auf allen drei Verwaltungsebenen bearbeitet, sind horizontal durch den Querschnittscharakter geprägt, so dass eine policy-übergreifende Abstimmung nötig ist, und es besteht ein formal institutionalisierter Zwang zur Kooperation im Mehrebenensystem (hoher Formalisierungsgrad der Verwaltungsverflechtung). Beispiele für die auftretenden Koordinationsprobleme sind die unzureichende Koordination z. B. der Sprachkursangebote zwischen Bund, Ländern und Kommunen, ein mangelhafter inner-/zwischenbehördlicher Datenaustausch, erhebliche Doppelarbeiten zwischen verschiedenen Organisationseinheiten sowie ein hoher Beantragungs- und Verwaltungsaufwand (vgl. Bogumil/Burgi u.a. 2018, Bogumil/Kuhlmann 2020). Möglichkeiten diese Koordinationsprobleme zu vermindern liegen

- in der Entflechtung (z. B. durch Verlagerung des Verwaltungsvollzugs der Integrationskurse und der berufsbezogenen Sprachförderung vom BAMF auf die Länder oder im Bereich der Digitalisierung durch stärkere Standardisierung und Zentralisierung),

- in der Optimierung von Verflechtungsstrukturen (z. B. Verbesserung des Datenaustausches zwischen Bund, Ländern und Kommunen durch mehr Standardisierung, Registermodernisierung und Überprüfung von Datenschutzregeln; stärkere Absprachen bei Fördermaßnahmen durch die Fachministerien, Aufbau Ministerien übergreifender Förderportale) oder

- in der Bündelung (z. B. Reform der unterschiedlichen Sozialleistungen wie SGB II, BAföG, Berufsausbildungsbeihilfe, Wohngeld, Pflegegeld in Richtung weniger Schnittstellen und Vorrangprüfungen, Vermeidung des Rechtskreiswechsels von Leistungen des AsylbLG / SGB III zum SGB II durch Abschaffung des AsylbLG und Öffnung des SGB II generell für Asylbewerber; prozessorientierte Bündelung von Verwaltungstätigkeiten durch Aufbau integrierter Verwaltungseinheiten für Migration und Integration sowohl auf der Ebene der Ministerien, der Bezirksregierungen als auch der Kommunen).

\subsubsection{Bürokratische Koordination}

Zur Bewältigung dieser überall verbreiteten Koordinationsprobleme in einer differenzierten, hoch-spezialisierten Verwaltung, hinter denen sich natürlich unterschiedliche inhaltliche, fachliche und politische Interessen und Präferenzen verstecken, gibt es allerdings seit langem bekannte klassische bürokratische Koordinationsmechanismen: 
- Federführung bedeutet, dass für jede Aufgabe eine und nur eine Organisationseinheit verantwortlich ist. Doppelzuständigkeiten darf es, so zumindest die Theorie, nicht geben. Jeweils eine Einheit ist dafür zuständig, dass die Aufgabe bewältigt wird, sie hat aber gleichzeitig dafür zu sorgen, dass andere Stellen, die daran ein Interesse haben oder von Entscheidungen betroffen sind, beteiligt werden. Dies geschieht zunächst im Rahmen der

- Mitzeichnung, d.h. Vorschläge, Verfügungen, Anordnungen, Pläne der Verwaltung müssen anderen, beteiligten Stellen zugeleitet werden und dürfen erst entschieden werden, wenn diese, eben durch ein schriftliches Kürzel, bestätigt haben, dass sie den Vorgang gesehen haben und damit einverstanden sind. Auch hier ist die Rationalität wiederum die Nachvollziehbarkeit der Verantwortlichkeit: Die Straßenbaubehörde soll nicht behaupten können, dass sie von der geplanten Kabelverlegung oder dem geplanten Naturschutzgebiet nichts gewusst habe. Umgekehrt wird die federführende Einheit gezwungen, andere zu beteiligen. Bei größeren Planungsvorhaben, bei denen interorganisatorische Abstimmungen notwendig sind, ist so gesetzlich vorgeschrieben, dass alle möglichen „Träger öffentlicher Belange“ zu beteiligen sind. ${ }^{60}$ Selbstverständlich können die umfassenden intra- oder auch inter-organisatorischen Konflikte nicht immer durch einfache schriftliche Mitzeichnung gelöst werden. Das nächste klassische bürokratische Koordinationsinstrument ist daher die

- Besprechung, in der die beteiligten Organisationen oder Organisationseinheiten versuchen, ihre unterschiedlichen Sichtweisen und Interessen miteinander zu vereinbaren. Wie bei den anderen Instrumenten auch, geht es immer gleichzeitig um Informationsbeschaffung und -verarbeitung und um Konfliktlösung und Konsensbildung. Jeder kennt wiederum die klassische und frustrierende Antwort, wenn bürokratische Akteure mal wieder nicht erreichbar sind: „Die Damen und Herren befinden sich gerade in einer Besprechung“. Abgesehen von der Frage, ob alle Besprechungen in Umfang, Dauer und Beteiligung notwendig sind, ist doch offenkundig, dass sie ein klassisches und unverzichtbares bürokratisches Instrument sind, auch in Zeiten von Videokonferenzen und Email. Wenn die Zahl der beteiligten oder interessierten Akteure zu umfangreich wird, gibt es schließlich die Möglichkeit der

- Anhörung, bei der nicht nur „formal“ Mitzeichnungsberechtigte an der Entscheidungsfindung beteiligt werden, sondern der Kreis anzuhörender Akteure und Interessen noch weiter ausgeweitet wird, in der Regel auch in die Richtung

60 Die Beteiligung der betroffenen Öffentlichkeit liegt dagegen nicht auf der Ebene der innerbürokratischen Verfahren und Prozesse, um die es hier geht. 
externer, also nicht- oder halb-öffentlicher Akteure (Kammern, Interessenverbände, Firmen, Bürgerinitiativen etc.).

\subsubsection{Positive und negative Koordination}

Die offenkundigen erheblichen Koordinationsprobleme innerhalb des öffentlichen Sektors zeigen sich nicht nur in Koordinationsfehlschlägen (die berühmte "Soda-Brücke", die einfach nur so da in der Landschaft herumsteht, ohne den eigentlich geplanten Anschluss), sondern insbesondere auch in zeitlichen Verzögerungen, z. B. bei der Bewilligung von Investitionsvorhaben. Kritisiert werden immer wieder die unzureichende gegenseitige Unterstützung und Integration staatlicher Vorhaben, etwa im Bereich der Regionalentwicklung, der Bildungspolitik oder der Kommunalpolitik, neuerdings ganz besonders im Bereich der Energie- oder Klimapolitik.

Darauf, dass dieses immer wieder beklagte Koordinationsversagen nicht einfach auf die Unfähigkeit oder Unwilligkeit der beteiligen Akteure zurückzuführen ist, hat Fritz Scharpf in einem berühmten Aufsatz über „Komplexität als Schranke politischer Planung“ (1973a) hingewiesen. Sein Ausgangspunkt ist, dass Politik und Verwaltung durch Differenzierung und Arbeitsteilung zwar eine der Umwelt entsprechende und angemessene Eigenkomplexität erreichen können (siehe oben „requisite variety“), dass es jedoch viel schwieriger ist, die realen Problemzusammenhänge und Interdependenzen, die es in der sozio-ökonomischen Umwelt der Verwaltung gibt, durch entsprechende Verknüpfungen und Mechanismen innerhalb des politisch-administrativen Systems zu reproduzieren. Regionalentwicklung hängt z. B. nicht nur von Wirtschaftsförderung ab, sondern von Verkehrspolitik, Arbeitsmarktpolitik, Umweltpolitik, Bildungspolitik usw.

Politische und gesellschaftliche Problemverarbeitung und damit administratives Handeln findet zunächst in den Basiseinheiten statt, also den Referaten oder Sachgebieten, die eben „federführend“ sind. Der Preis für deren Spezialisierung und Expertise ist die selektive Perzeption, d. h. jede Einheit konzentriert sich auf ihre Zuständigkeiten und nimmt Probleme außerhalb kaum oder überhaupt nicht wahr oder hält sie zumindest für weniger wichtig. Das zentrale Anliegen der Straßenbauer ist nun einmal der Bau von Straßen, und nicht die Erhaltung von Feuchtbiotopen für Krötenwanderungen. Aus diesem Grund sind interdependente Probleme schwer zu bearbeiten und (mögliche) negative Folgewirkungen des eigenen Handelns in anderen Bereichen werden oft nicht gesehen oder bewusst ignoriert.

Eine mögliche Lösung dieses Dilemmas besteht darin, Entscheidungen „hochzuzonen“, sie zu zentralisieren. Dies führt aber in der Regel eher zu einer Verschlechterung als zu einer Verbesserung der Entscheidungsqualität, denn „auf dem Dienstweg " nach oben findet nun einmal eine notwendige Informationsverdichtung und damit ein Informationsverlust statt. Wenn man interdependente Probleme 
lösen will, braucht man aber mehr, nicht weniger Informationen. Übertriebene Zentralisierung und Hierarchisierung führt also zu Informationsverlust und vor allem zu einer Überlastung der Hierarchie sowohl bezüglich ihrer Informationsverarbeitungs- wie auch ihrer Konfliktlösungskapazität. Wenn zu viele Entscheidungen „nach oben“" abgeben werden, wird in aller Regel der Zeitbedarf steigen und die Qualität von Entscheidungen sinken.

Eine andere Strategie ist, die dezentralen Koordinationskapazitäten von Organisationen zu erhöhen. Hier spielt die Unterscheidung von negativer und positiver Koordination eine Rolle:

- Negative Koordination bezeichnet die normale Praxis: Die Initiative zur Problemverarbeitung geht von einer spezialisierten Einheit aus und ist dieser zugeordnet, und diese ist vorrangig auf das eigene Problem fixiert, ansonsten interessiert nur, inwieweit andere Bereiche negativ durch vorgesehene Lösungen und Maßnahmen betroffen sind. Um die eigene Problemlösungsfähigkeit nicht zu früh einzuschränken, werden andere Bereiche und Interessen daher so spät wie möglich einbezogen, damit sie die eigene Problemlösung nicht stören, typischerweise mit dem Instrument der Mitzeichnung. Es wird also abgeklärt, inwieweit andere Einheiten mit einer Lösung „nicht leben“ können, und nicht, inwieweit sie ggf. selbst etwas zur Lösung des Problems beitragen könnten.

- Positive Koordination will genau dies erreichen: Auf der Grundlage einer Analyse des gesamten interdependenten Problemzusammenhangs sollen Maßnahmen aus unterschiedlichen Bereichen ausgewählt werden, die einander unterstützen und gemeinsam zur Problemlösung beitragen. Dies bedeutet aber, dass in allen voneinander abhängigen Entscheidungsbereichen alle infrage kommenden Entscheidungsalternativen gemeinsam und gleichzeitig zur Disposition gestellt werden müssen.

Dass reale Verwaltungen in aller Regel negative Koordination vorziehen ist allerdings nicht einfach auf deren Unfähigkeit, Bequemlichkeit oder das Vorherrschen von Fachidioten zurückzuführen, sondern kann mit den entstehenden „Koordinationskosten" erklärt werden. Dazu benutzt Scharpf ein ganz einfaches Zahlenbeispiel: In Abbildung 44 haben wir es mit folgender Situation zu tun: Insgesamt gib es drei Entscheidungsbereiche (z. B. Referate oder Ämter, n=3) und eines der Ämter muss zwei Handlungsalternativen mit den anderen beiden abklären $(\mathrm{a}=2)$. Hier sind also vier gegenseitige Abhängigkeiten oder Relationen zu prüfen. Wenn im gleichen Beispiel jede Einheit zwei Alternativen präsentiert hätte, deren gegenseitige Abhängigkeiten zu prüfen wären, wären dies schon 24 Relationen oder Koordinationsschritte (Abbildung 45). 


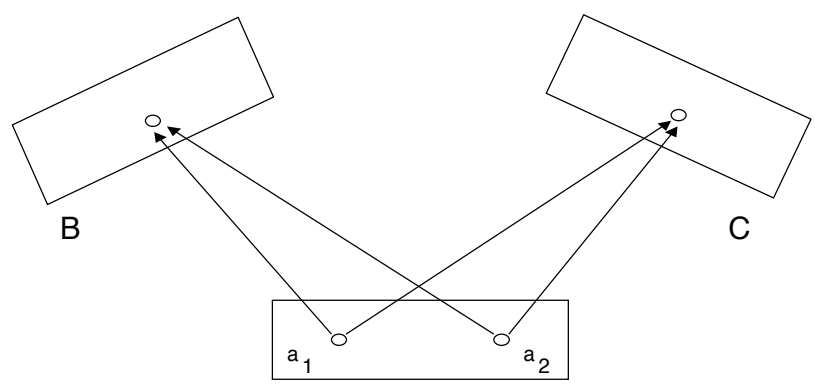

A

$$
\mathrm{R}_{\mathrm{n}}=(\mathrm{n}-1){ }^{*} \mathrm{a}
$$

Abb. 44 Negative Koordination

Quelle: Scharpf 1973a

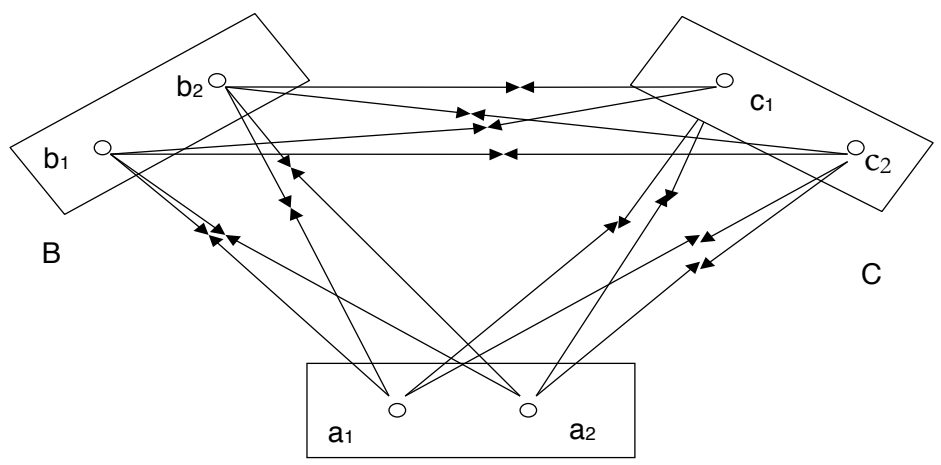

A

$$
\mathrm{R}_{\mathrm{n}}=\mathrm{n} *(\mathrm{n}-1){ }^{*} \mathrm{a}^{2}
$$

Abb. 45 Positive Koordination

Quelle: Scharpf 1973a

Die Zahl der jeweils notwendigen Prüfungen kann durch einfache Formeln ermittelt werden (auf den „Beweis“ dieser Formeln wird hier verzichtet). Dies bedeutet aber folgendes: Wenn wir es statt nur mit drei mit fünf Einheiten $(n=5)$ zu tun hätten, die jeweils drei Alternativen ins Gespräch bringen $(a=3)$, steigt der Koordinations- 
bedarf im Rahmen der negativen Koordination auf immerhin zwölf zu prüfende Möglichkeiten, nämlich $(5-1)^{*} 3=12$. Bei positiver Koordination wären dies aber bereits $5^{\star}(5-1)^{\star} 9=180$ Alternativen. Schon diese rein schematische Betrachtung verdeutlicht, warum Organisationseinheiten, wann immer es möglich ist, den Mechanismus der negativen Koordination wählen werden: der normativ vorzuziehende Weg der positiven Koordination führt sehr schnell zu einer Überlastung der Informations- und Konfliktverarbeitungskapazitäten von Organisationen. In den Worten von Scharpf:

„Selbst wenn es im Entscheidungsprozess keine verfestigten Interessen und auf Machtpotentiale gestützten Veränderungswiderstände gäbe, wenn also alle Beteiligten prinzipiell innovationsbereit und für rationale Argumentation offen wären, selbst dann müsste der Versuch der simultanen Problematisierung und positiv koordinierten Veränderung interdependenter Entscheidungsbereiche (...) notwendigerweise in der Frustration des totalen Immobilismus enden" (Scharpf 1973a, S. 93).

Auch positive und negative Koordination sind offenkundig „Idealtypen“, die in der Realität in reiner Form kaum vorkommen. Allerdings haben empirische Untersuchungen immer wieder gezeigt, dass das alltägliche Geschäft bürokratischer Organisationen, und damit auch der deutschen Verwaltung, immer noch durch die Merkmale negativer Koordination gekennzeichnet ist, gerade auch bei neuartigen Problemen wie der Klimapolitik, und selbst wenn zur Koordination verstärkt interministerielle Arbeitsgruppen eingesetzt werden (vgl. Radtke/Hustedt/Klinnert 2016, Hustedt/Danken 2017).

Tatsächlich gibt es eine spezifische Form der innerbürokratischen Koordination und Abstimmung, die verhältnismäßig gut funktioniert, die aber auch alles andere als unproblematisch ist, nämlich die von Frido Wagener zunächst als „vertikale Ressort-Kumpanei“ (1975, S. 134) oder, etwas freundlicher, als „Fachbruderschaften“ (1979, S. 238ff.) bezeichnete Koordinierungsbürokratie. Gemeint ist damit die vertikale, nicht nur organisations- sondern auch ebenenübergreifende, Zusammenarbeit bestimmter Fachverwaltungen. Im Kern geht es darum, dass sich z. B. zwischen den zuständigen Behörden etwa im Bereich Agrar-, Verkehrs- oder auch Umweltverwaltung notwendigerweise über die Jahre enge Kontakte entwickeln, die sowohl lokale (Kommunalverwaltung), regionale (Mittelinstanzen), landesweite (Landesministerien) und bundesweite Organisationen umfassen können und inzwischen bis auf die EU-Ebene ausgedehnt werden. Das, worauf sich diese „Fachbrüder und -schwestern“ aus ihrer sektoralen, „fachlichen“ Sicht geeinigt haben, kann oft nur sehr schwer auf der jeweils politisch zuständigen Ebene verändert werden. Etwas zugespitzt formuliert: Wenn sich die Verkehrsplaner im Bundesministerium mit ihren Kollegen auf Landesebene, und die wiederum mit den Kollegen im Regie- 
rungspräsidium und im Kreis auf den Ausbau einer bestimmten Straße geeinigt haben, und sich zudem auch einig sind, dass dabei bestimmte Standards unbedingt einzuhalten sind (etwa Fahrbahnbreite, Kurvenradien), dann ist dies von den jeweils politisch verantwortlichen Gremien kaum noch zu verändern. Sobald der Kreistag (oder etwa eine andere Fachbehörde) versuchen wird, Einfluss auf diese Maßnahme zu nehmen, kann ihnen vorgehalten werden, dies sei bereits mit den anderen Ebenen abgestimmt, und der Versuch dieses Ergebnis infrage zu stellen würde ggfs. das gesamte Projekt torpedieren.

Besonders problematisch wird diese vertikale Koordination, wenn sie mit finanziellen Anreizen verbunden ist. Die inhaltliche Politikverflechtung der Fachverwaltungen wird dann durch eine ebenenübergreifende Mischfinanzierung unterstützt, die dazu führt, politische Handlungsspielräume weiter einzuschränken, denn schließlich will man öffentliche Mittel möglichst "mitnehmen“ und nicht „verschenken“. Wagener weist darauf hin, dass es dabei subjektiv nicht bösartig zugeht, sondern durchaus löblich, denn es geht darum, die eigene fachliche Aufgabenwahrnehmung zu optimieren. Im Ergebnis führt dies allerdings zu einer politisch unkontrollierten Selbstbestimmung der Fachbürokraten. In den Worten von Frido Wagener:

„In den Ministerien von Bund und Ländern und in den Spitzenstellungen der Großstädte und Landkreise ist der öffentliche Dienst heute jedenfalls weidlich damit beschäftigt, den Standard der öffentlichen Aufgabenerfüllung und teilweise auch die Erfindung neuer öffentlicher Aufgaben mit Hilfe von Gesetzen, Erlassen und Programmen, insbesondere aber durch Geld, an dessen Annahme man Bedingungen knüpft, zu steuern. (...) der besonders gute Ministerialbeamte hat (...) die Formulierung schon im Vorfeld der Entscheidung soweit vorbesprochen, herunterkoordiniert, abgestimmt und mit ein klein wenig Änderungsstoff für die partout nicht zu überzeugenden Politiker angereichert, dass im Ergebnis genau das aus dem gesetzgeberischen Ratifizierungsprozess herauskommt, was die Ministerialbürokraten für angemessen und machbar halten. Dies ist gewöhnlich nicht wenig. Es ist genau geregelt und entspricht einem hohen Standard“ (Wagener 1979, S. 242).

Also gerade die zu perfekte vertikale Koordination fachlicher Standards und Interessen kann zu kontraproduktiven Ergebnissen führen. Die Schlussfolgerungen von Frido Wagener aus dem Jahre 1979 sind an Deutlichkeit kaum zu übertreffen, auch wenn sie die Möglichkeiten politischer Steuerung der Ministerien (vgl. unten 4.4) vermutlich unterschätzen:

„Es ist festzuhalten, dass die Leitungsebene des öffentlichen Dienstes im bundesdeutschen Staat der Gegenwart ganz im Gegensatz zum Verfassungsverständnis unserer Staatsrechtslehre Art und Umfang der öffentlichen Aufgabenerfüllung nicht etwa 
nur mitbestimmt, sondern in pragmatischer Illegalität weitgehend selbst bestimmt“ (ebd., S. 243).

\subsubsection{Bürokratiekritik und Bürokratieabbau}

Wie erwähnt ist Bürokratiekritik erheblich älter als die formale Definition bürokratischer Merkmale durch Max Weber. Bürokratiekritik zeichnet sich zunächst aus durch einen ungemein diffusen Charakter (vgl. auch Derlien/Böhme/Heindl 2011, S. 29ff.). Es gibt vermutlich in Deutschland, aber das gilt sicherlich auch für andere Länder, kaum eine einfachere und billigere Art und Weise sich öffentlichen Beifall zu sichern als „die Bürokratie“ anzuprangern. Ein kurzer Blick in die aktuelle Presse zeigt dies. Dabei werden sehr unterschiedliche Phänomene kritisiert, z. B.

- bürokratische Sprache: gestelzte, unverständliche bis absurde Vorschriften, Formulare und Bescheide;

- bürokratisches Verhalten: Unpersönlichkeit, Unfreundlichkeit, langsame und schwerfällige Bearbeitung, Verantwortungsscheu, Kontrollfixierung;

- bürokratische Persönlichkeit: Rigidität, Dogmatismus, Risikovermeidung, Zielverschiebung (Mittel wird zum Zweck);

- bürokratische Vorschriften: Verrechtlichung und Überregelung, für die Betroffenen nicht einsehbare und nachvollziehbare Regelungen, die Eigeninitiative einengen, Kosten verursachen und Aktivitäten verzögern;

- bürokratische Geschäftsprozesse und Organisationsstrukturen: Zuständigkeitswirrwarr, negative Auswirkungen der selektiven Problemwahrnehmung, Überbetonung formaler Richtigkeit gegenüber Effizienz und Effektivität;

- bürokratische Verselbstständigung: unkontrollierte Machtausübung, fehlende politische Loyalität, Klientelismus usw.

Offenkundig beinhalten alle diese Kritikpunkte viele Stereotypen. Aus Umfragen wissen wir, dass „der Bürokratie“ alle diese negativen Merkmale zugeschrieben werden, dass aber, je konkreter gefragt wird, die Befragten ihre praktischen Erfahrungen mit öffentlichen Bürokratien und Bürokraten viel positiver bewerten. Insgesamt sind die Einstellungen der Bevölkerung gegenüber der Bürokratie widersprüchlich, man könnte sogar sagen gelegentlich schizophren: Zwei Drittel der Bevölkerung und mehr sind im Allgemeinen mit der Verwaltung zufrieden, der Anteil, der über positive persönliche Erfahrungen berichtet, ist teilweise noch höher. Zugleich werden bestimmte Behördenmerkmale und administrative Verfahren von $80 \%$ und mehr der Befragten kritisiert (Grunow/Strüngmann 2008 m.w.A.). 
Klagen über bürokratische Hemmnisse und Überregelung gehören seit Jahren, wenn nicht Jahrzehnten, zum guten Ton - und das nicht nur in Deutschland (die ersten Entbürokratisierungskommissionen gab es z.B. schon in der Weimarer Republik und in der Bizone, vor der Gründung der Bundesrepublik). Auf allen staatlichen Ebenen gibt es daher seit Jahren eine beinahe unüberschaubare Anzahl von Initiativen zum „Bürokratieabbau“, zur „Entbürokratisierung“ und „Deregulierung" (vgl. Jann/Wegrich 2008). Gleichzeitig ergab eine Unternehmensbefragung, dass im Jahr $199458 \%$ die Belastung durch staatliche Bürokratie als hoch oder sehr hoch einschätzten, zehn Jahre später gaben dies $79 \%$ an (Kayser u. a. 2004). War die Belastung durch staatliche Bürokratie tatsächlich in zehn Jahren um mehr als ein Drittel gestiegen?

Tatsächlich ist die aktuelle Diskussion stark durch Befunde „gefühlter Bürokratie" bestimmt, die kaum mit objektiven Daten unterlegt werden (vgl. zum Folgenden ausführlich Jann/Wegrich/Tiessen 2007, Jann/Wegrich 2008). In einer aktuellen Studie des IfM (Institut für Mittelstandsforschung) kritisieren z. B. 78 \% der Unternehmen die Regelungsdichte und $59 \%$ stellen den Sinn vieler Vorschriften generell infrage. Aber die Autoren der Studie weisen ausdrücklich darauf hin, dass fast alle Unternehmen den Bürokratie-Begriff sehr weit fassen und darunter z. B. auch halb-öffentliche (z. B. durch Berufsgenossenschaften) oder sogar privatwirtschaftliche Regulierung (in Verträgen) verstehen (Holz et.al. 2019).

Die Bundesregierung lässt seit 2015 alle zwei Jahre die Zufriedenheit sowohl der Bürgerinnen und Bürger wie der Unternehmen mit der deutschen Bürokratie ermitteln, mit bemerkenswerten Ergebnissen (zu finden unter www.amtlich-einfach.de). So sind insgesamt $83 \%$ der Unternehmen mit der öffentlichen Verwaltung eher oder sehr zufrieden und mehr als die Hälfte der Unternehmen empfanden ihre Behördenkontakte als sehr oder eher einfach. Seit 2015 ist das Vertrauen in Behörden sogar gestiegen, auf einer Skala von -2 bis +2 liegt es 2019 bei 1,1 (wenn man diese Skale auf die normale Notenskala von 1 bis 5 übertragen würde, wäre dies eine Note von 1,9 - also besser als gut). Hilfsbereitschaft und Fachkompetenz der Behörden werden auf der Ursprungsskala mit 1,3 bewertet, eGovernment und Verständlichkeit schneiden mit 0,5 am schlechtesten ab, bei Unbestechlichkeit und Diskriminierungsfreiheit ist die Bewertung 1,9, also nahe am Höchstwert von 2,0.

Die Zufriedenheit mit der deutschen Verwaltung ist also höher als man denkt. Dennoch reißt die Kritik an der Bürokratie nicht ab, wie ein kursorischer Blick in die Zeitungen bestätigt, zumal viele Menschen durchaus negative Erfahrungen mit längeren Wartezeiten, kaum verständlichen Formularen oder ,übergründlich “ prüfenden Verwaltungsmitarbeitern machen. Das hat aber vor allem damit zu tun, dass diese Kritikpunkte keinesfalls alle auf der gleichen Ebene liegen und schon gar nicht alle auf eine einzige Ursache zurückzuführen sind. Es ist daher hilfreich 
und notwendig zu differenzieren: Dabei kann man mindestens vier Dimensionen der Bürokratiekritik unterscheiden (Mayntz 1980b; Jann/Wegrich 2008):

- die Aufgabenebene (Umfang der vom Staat bzw. von der öffentlichen Verwaltung wahrgenommen Aufgaben),

- die Regulierungsebene (Anzahl und Dichte der Regulierungen bzw. der darin festgelegten materiellen Standards, Qualität der Regulierungen),

- die Organisationsebene (Anzahl staatlicher Behörden, horizontale und vertikale Koordination, Merkmale der Ablauforganisation und des Personals etc.) und

- die politische Ebene (Verselbständigung der Bürokratie, politische Steuerung der Verwaltung etc., siehe ausführlich unten 4.3).

\subsubsection{Aufgaben: zu viel Staat}

Ein erheblicher Teil der gängigen Bürokratiekritik ist eigentlich eine Kritik des modernen Interventionsstaates. Diese Ebene der Bürokratiekritik beanstandet im Kern, dass es zu viele staatliche Aktivitäten und Interventionen in zu vielen Bereichen gibt. Dies ist offenkundig eine alte und kontroverse, aber auch vollkommen legitime und notwendige politische Diskussion. Dennoch wird hier oft der Sack „Bürokratie“ geschlagen, und eigentlich der Esel Sozialstaat, oder präziser sozialer Rechtsstaat gemeint. Hierunter fällt z. B. die Kritik

- am Wachstum und Umfang neuer staatlicher Aufgaben - also etwa Gleichstellungs-, Klima- oder Sozialpolitik - allerdings hat das vorrangig etwas mit politischen Schwerpunkten zu tun, und weniger mit Bürokratie;

- an der wachsenden Staatsquote, (Bürokratisierung als Finanzproblem);

- am Wachstum der öffentlichen Verwaltung (Bürokratisierung als Personalproblem).

Bezüglich der „Bürokratisierung als Finanzproblem“ zeigt sich in Deutschland, dass staatliche Ausgaben und Einnahmen (Staats- und Abgabenquote) europaweit im durchschnittlichen bis unterdurchschnittlichen Bereich liegen. Die Ausgaben des deutschen Staates gemessen am BIP, also die sogenannte Staatsquote, lag z. B. 2017 mit $44 \%$ unter dem EU-27 Schnitt von $46 \%$ (vgl. Abb. 8). Allerdings stellt sich die Frage, ob legitime Klagen über „zu viel staatliche Ausgaben“ überhaupt etwas mit Bürokratie zu tun haben und nicht vielmehr mit sozialstaatlicher Schwerpunktsetzung. Immerhin machen Sozialausgaben inzwischen ca. $41 \%$ des Bundeshaushalts aus; wenn die Ausgaben für Zinsen und Verteidigung dazu kommen, bleiben für sämtliche übrigen staatlichen Ausgaben auf Bundesebene gerade noch gut $30 \%$ übrig. Politische Kontroversen über Umfang und Schwerpunkte staatlicher Auf- 
gaben und Ausgaben sind notwendig und legitim, aber sie sind etwas anderes als Bürokratiekritik.

Hinsichtlich des Personalumfangs des öffentlichen Dienstes (öffentlich Beschäftigte) gibt es keine Anzeichen dafür, dass es in Deutschland einen im internationalen Vergleich „aufgeblähten öffentlichen Dienst" gibt, wie bereits in Kapitel 3.6.2 ausgeführt. Nach der Wachstumsphase in den siebziger Jahren ist vor allem seit den neunziger Jahren ein anhaltender Verschlankungsprozess zu beobachten, so dass der öffentliche Dienst in Deutschland im internationalen Vergleich eher unterdurchschnittlich groß ist. Der Anteil der öffentlichen Beschäftigten an den Gesamtbeschäftigten liegt in Deutschland bei ca. $11 \%$, gegenüber 17,7\% im OECD-Durchschnitt, und somit im unteren Drittel der OECD (2019). Ein „aufgeblähter öffentliche Dienst" ist sicher nicht das zentrale Bürokratieproblem. Eher stellt sich die Frage, und wird auch öffentlich diskutiert, ob der Personalabbau der letzten Jahrzehnte zu umfangreich war, ob das Personal im öffentlichen Dienst zukunftsfähig ausgebildet ist und ob unter den Bedingungen des bestehenden Dienstrechts mit Humanressourcen intelligent umgegangen wird.

Im Gegensatz zu diesen empirischen Daten bezogen auf die jüngeren Entwicklungen in der Deutschland, behauptet die ökonomische Theorie der Bürokratie unverdrossen (grundlegend Downs 1966, Niskanen 1971, als Übersicht Braun 2013), dass ein erheblicher Anteil des Wachstums öffentlicher Budgets, Regelungen und Personals ,endogen“, also durch interne Merkmale bürokratischer Organisationen erklärt werden kann. Verwaltungsangehörige werden nicht als uneigennützige Diener des Allgemeinwohls aufgefasst, sondern als ganz normale opportunistische Nutzenmaximierer, die vor allem eigennützige Ziele wie Einkommen, Prestige, Sicherheit oder Macht verfolgen. Aufgrund der Merkmale bürokratischer Organisationen, insbesondere der asymmetrischen Informationsverteilung und der beschränkten Kontrollkapazität der Vorgesetzten und der Politik, nutzen die Mitglieder der Bürokratie ihre Handlungsspielräume für ihre eigenen Interessen. Dies führt u. a. zu einem Überangebot öffentlicher Güter und Dienstleistungen und zu überhöhten Kosten. Bürokraten agieren als „Budgetmaximierer“, indem sie aus eigennützigen Gründen versuchen, die Anzahl der ihnen unterstellten Mitarbeiter oder das von ihnen kontrollierte Budget zu maximieren. Wenn, wie es früher z. B. in der bürokratisierten Reichs- oder Bundesbahn üblich war, die Besoldung von Stationsvorstehern von der Anzahl ihrer Untergebenen abhängig ist, werden diese versuchen, deren Anzahl zu erhöhen. Mit anderen Worten wachsen Bürokratien nach dieser Erklärung nicht vorrangig aufgrund externer Anforderungen, sondern aufgrund ihrer internen Strukturen. Die erste Formulierung dieses „Gesetzes vom Wachstum der Bürokratie“ stammt von dem englischen Wissenschaftler 
und Satiriker C. Northcote Parkinson und wurde als Parkinsons Gesetz bekannt (Parkinson 1957).

\subsubsection{Regulierung: Normenflut, Verrechtlichung und Informationskosten}

Ein weiterer Schwerpunkt der modernen Bürokratiekritik ist die Kritik staatlicher Regulierung gegenüber Wirtschaft und Gesellschaft. Hier geht es zum einen um die Reduzierung von Regeln und Vorschriften, um De-Regulierung. Dies ist die Kritik an der Gesetzes- und Verordnungsflut, an den „überflüssigen“ oder „unnützen“ Gesetzen und Vorschriften. Diese Diskussion wird in Deutschland seit den siebziger Jahren unter den Stichworten der Überregelung, Normenflut oder Verrechtlichung geführt (Voigt 1993).

Im Kern geht es hier zum einen um den quantitativen Umfang der regulativen Aktivitäten des Staates, womit sämtliche rechtliche Regelungen aller möglichen staatlichen Steuerungsaktivitäten gemeint sind, unabhängig von den dabei zur Anwendung kommenden Instrumenten, also etwa Ge- und Verbote, aber auch finanzielle Anreizprogramme, Besteuerung oder Information. Davon zu unterscheiden, aber oft in einem Atemzug genannt, ist die materielle Regelungsebene. Gemeint sind damit die materiellen Standards etwa im Umwelt-, Arbeits- oder Verbraucherschutz. Hier geht es um den Umfang des Kündigungsschutzes, die inhaltlichen Kriterien im Immissionsschutz, Qualitätskriterien für Nahrungsmittel oder sogar um das Recht, bei staatlichen Planungen beteiligt zu werden und sich zu wehren. Alles dies sind politisch hoch kontroverse Fragen.

Die Abschaffung „bürokratischer Hemmnisse“ im Arbeits- oder Umweltrecht hat vor allem etwas damit zu tun, welche Rechte wie geschützt werden sollen und für wie schützenswert man diese Rechte im Abwägungsprozess hält. Auch unser Steuerrecht ist nicht so überaus kompliziert, weil wildgewordene, hyperaktive Beamte im Finanzministerium außer Kontrolle geraten sind, sondern weil es sehr gut informierte und aktive Interessengruppen gibt, denen es gelungen ist, ihre (durchaus legitimen) Interessen in einzelnen Bereichen des Steuerrechts durchzusetzen. Und auch die Änderung der Handwerksordnung und die Abschaffung des Meisterzwangs ist, wie gut zu beobachten war, ein politischer Kraftakt, kein bürokratischer, der letztendlich dann auch weitgehend gescheitert ist. Alle diese Interessen sind nicht bürokratisch begründet, sondern allein politisch. Im Prinzip ähnelt die ,Normenflut' der ewigen Debatte um überflüssige Subventionen. Generell ist die Öffentlichkeit davon überzeugt, es gäbe viel zu viele Subventionen, wenn aber eine konkret abgeschafft werden soll, gibt es erheblichen politischen Widerstand. Genauso ist auch staatliches Sparen generell überaus populär, allerdings nicht, wenn konkret irgendwo gespart werden soll. 
Davon zu unterscheiden ist zum anderen die Kritik der Ausgestaltung von Regulierungen, im Sinne von (ausführlich Mayntz 1980b)

- Umfang,

- Dichte,

- Genauigkeit,

- Kosten,

- Effektivität und

- Problemlösungsfähigkeit von staatlichen Regelungen.

Hier wird im Kern angenommen, dass das gleiche Niveau der Zielerreichung staatlicher Vorschriften (z. B. Einhaltung bestimmter Sicherheitsstandards) mit geringeren administrativen Lasten, d.h. Kosten für die Betroffenen (und die Verwaltung), zu erreichen ist. Das aktuelle Schlagwort ist hier nicht De-Regulierung, sondern „better regulation“ (als Übersicht Wegrich 2011, Jantz/Veit 2019), und der zentrale Ansatzpunkt sind Informations- und Erfüllungsaufwand, die sich aus rechtlichen Regelungen ergeben, also etwa welche Daten gegenüber staatlichen Stellen wie oft in welchem Detaillierungsgrad nachgewiesen werden müssen.

Ent-Bürokratisierung im Sinne von Vorschriftenreduzierung und Rechtsvereinfachung hat in Deutschland eine lange Tradition. Zu erinnern ist an eine große Entbürokratisierungswelle Ende der siebziger, Anfang der achtziger Jahre sowohl in den Bundesländern als auch etwas später auf Bundesebene („Waffenschmidt-Kommission"), in der es um Verwaltungsvereinfachung, Beschleunigung von Genehmigungsverfahren und Anfang der neunziger Jahre um Erleichterung von Gewerbeansiedlung in den neuen Bundesländern ging. Es gab den Sachverständigenrat „Schlanker Staat“, der einen „Deregulierungsbericht“ vorlegte oder auch den „Masterplan Bürokratieabbau" der zweiten Regierung Schröder. 2006 hat dann die Große Koalition nach niederländischem Vorbild die Institutionalisierung eines unabhängigen Gremiums zur Bürokratiekostenmessung und -reduktion (Nationaler Normenkontrollrat) sowie die Übernahme des auch in den Niederlanden entwickelten Standardkosten-Modells (SKM) zur Messung der administrativen Belastungen durchgesetzt (als Überblick Jann/Jantz 2008a, Jantz/Veit 2019). Die Bundesregierung hatte sich dabei verpflichtet, die mit bestehenden Informationspflichten verbundenen Belastungen messbar zu senken (bis 2011 um 25\%) und neue Informationspflichten für Bürger, Unternehmen und Verwaltung zu vermeiden, und hat diese Ziele auch tatsächlich erreicht. Alle neuen Gesetzesvorhaben der Bundesregierung werden seitdem von den Ressorts vorab auf ihre Bürokratielasten geprüft, und diese Prüfung wird vom Normenkontrollrat überwacht (siehe ausführlich zu den Maßnahmen und ihren Ergebnissen Kapitel 5.2.2). 


\subsubsection{Verfahren: Vor- und Nachteile bürokratischer Organisation}

Schließlich geht es unter dem Stichwort der Bürokratiekritik um die interne Arbeitsweise von Behörden und öffentlichen Organisationen, also um übertriebene Formalisierung, Hierarchisierung, aber auch Arbeitsteilung und Professionalisierung. Kritisiert wird u. a. eine zu langsame und schwerfällige Bearbeitung, interne Koordinationsprobleme zwischen und in Behörden und mangelhaftes Kostenbewusstsein, und gegenüber Kunden und Klienten, Bürgerferne, Unpersönlichkeit, mangelnder Dienstleistungs- und Kundenorientierung, die bekannt-berüchtigte Verwaltungssprache und generell eine unzureichende Information und Transparenz der Verwaltung.

Auf der Grundlage der Erfahrungen der Entbürokratisierungskommissionen der Länder der siebziger und achtziger Jahre hatte Wolfgang Seibel bereits 1986 darauf hingewiesen, dass diese Dysfunktionen bürokratischer Organisation nur die Kehrseite durchaus positiver und erwünschter Funktionen sind, sowohl für die Bürgerinnen und Bürger wie für den Staat oder die Politik:

- Die strikte bürokratische Arbeitsteilung hat für den Staat den Vorteil der dauerhaften spezialisierten und kompetenten Aufgabenwahrnehmung, für den Bürger wird so die verlässliche Zuständigkeit einer bestimmten Einheit garantiert. Allerdings ist diese Arbeitsteilung auch verantwortlich für die beschriebenen Phänomene der selektiven Problemperzeption und die damit verbundene vorherrschende negative Koordination, während sie sich für den Bürger oft als undurchschaubares Zuständigkeitslabyrinth darstellt.

- Die klassische Regelbindung ermöglicht auf der Seite des Staates erst die kontinuierliche und verlässliche Steuerung der Verwaltung, für den Bürger ist sie die Garantie der Berechenbarkeit und möglichen Kontrolle des Verwaltungshandelns. Umgekehrt führt sie auch intern zu Verrechtlichung und Überregelung bis hin zu einer „pragmatischen Vorschriftenreduktion im Vollzug“ (Frido Wagener), bei der sich die Verwaltung aus der Überfülle relevanter Vorschriften diejenigen heraussuchen kann, die gerade „passen“ (Wagener 1979). Für den Bürger ist die Regelbindung Ursprung bürokratischer Komplexität, Distanz und Abgehobenheit - bis hin zu den Verhaltensweisen der „bürokratischen Persönlichkeit“ (Rigidität, Zielverschiebung etc.), die letztendlich zu einer Verrechtlichung aller möglichen Lebensbereiche führt und die „Herrschaft der Bürokratie“ stabilisiert.

- Hierarchie ist für den Staat eine weitere notwendige Bedingung der Steuerung von Verwaltungseinheiten, und für den Bürger garantiert sie klare Verantwortlichkeitsketten und Einspruchsmöglichkeiten. Innerhalb der Bürokratie kann sie allerdings auf der einen Seite bei den Beschäftigten zu Motivations- 
verlusten, Verantwortungsscheu und Risikovermeidung führen, andererseits $\mathrm{zu}$ Informations- und Konfliktüberlastung bei den Vorgesetzten. Zusammen mit Regelbindung liegen hier einige der Ursachen für langsame, schleppende Bearbeitung und mangelhafte flexible Berücksichtigung spezifischer Bürgerinteressen („Bürgerferne“).

- Professionalität des Personals garantiert für den Staat wie für die Bürger die notwendige Fachkompetenz bei der Erledigung öffentlicher Aufgaben, aber die Kehrseite ist hier auf der Seite des Staates die Überbetonung bis hin zur Verabsolutierung professioneller Interessen, für die Bürger Überheblichkeit und professionelle Besserwisserei (die Bürger wissen nicht, was gut für sie ist).

\begin{tabular}{|c|c|c|c|c|}
\hline \multirow[t]{2}{*}{$\begin{array}{l}\text { Organisations- } \\
\text { merkmale }\end{array}$} & \multicolumn{2}{|c|}{ Funktion } & \multicolumn{2}{|c|}{ Dysfunktion } \\
\hline & Für den Staat & Für den Bürger & Für den Staat & Für den Bürger \\
\hline Arbeitsteilung & $\begin{array}{l}\text { Spezialisierung, } \\
\text { Kompetenz- } \\
\text { garantie }\end{array}$ & $\begin{array}{l}\text { Zuständigkeits- } \\
\text { garantie }\end{array}$ & $\begin{array}{l}\text { Selektive } \\
\text { Perzeption/ } \\
\text { Negativ- } \\
\text { koordination }\end{array}$ & $\begin{array}{l}\text { Zuständigkeits- } \\
\text { labyrinth }\end{array}$ \\
\hline Regelbindung & $\begin{array}{l}\text { Steuerungs- } \\
\text { entlastung }\end{array}$ & Berechenbarkeit & $\begin{array}{l}\text { Kontroll- } \\
\text { überlastung }\end{array}$ & $\begin{array}{l}\text { Verrechtlichung } \\
\text { der Lebenswelt, } \\
\text { Verfahrens- } \\
\text { komplexität }\end{array}$ \\
\hline Hierarchie & $\begin{array}{l}\text { Steuerung und } \\
\text { Kontrolle }\end{array}$ & $\begin{array}{l}\text { Verantwort- } \\
\text { lichkeit }\end{array}$ & $\begin{array}{l}\text { Motivations- } \\
\text { verlust, Kon- } \\
\text { fliktverdichtung }\end{array}$ & $\begin{array}{l}\text { Einschränkung } \\
\text { dezentraler } \\
\text { Flexibilität }\end{array}$ \\
\hline Professionalität & Fachkompetenz & Fachkompetenz & $\begin{array}{l}\text { Betriebs- } \\
\text { blindheit }\end{array}$ & „Expertokratie“ \\
\hline
\end{tabular}

Abb. 46 Funktion und Dysfunktion bürokratischer Organisation

Quelle: eigene Darstellung nach Seibel 1986

Es ist allerdings fraglich, inwieweit „normale“ Bürgerinnen und Bürger wirklich unter der deutschen Bürokratie leiden, jenseits aller Vorurteile und Stereotypen, insbesondere, wenn man davon ausgeht, dass diese „normalen Bürger“ durchschnittlich nur ca. zwei bis drei Behördenkontakte jährlich aufweisen. Außer in bestimmten „Lebenslagen“ (Umzug, Autokauf, Hausbau, Geburt) ist die Bürokratie vor allem Ansprechpartner bestimmter ausgewählter Gruppen (vor allem der sozial Schwachen) und ansonsten vor allem des "großen Publikums" der Unternehmen und Freiberufler. Aus diesem Grund richten sich die aktuellen Bestrebungen, ad- 
ministrativen Belastungen zu messen und zu reduzieren, zum einen vor allem an bestimmte Lebenslagen und Betroffene (etwa die Eltern behinderter Kinder), zum anderen an die Belastung von Unternehmen und Freiberuflern (Jantz/Veit 2019; siehe auch die jährlichen Berichte des Normenkontrollrats unter https://www. normenkontrollrat.bund.de).

Hinsichtlich der Bürokratieproblematik im Sinne allgemeiner „bürokratischer Organisation und Verfahren" (also Kundenzufriedenheit, Schnelligkeit, Qualität etc., s. o. 4.3.1) zeigen vorliegende Untersuchungen, ungeachtet der problematischen Umsetzung der Reformen des Neuen Steuerungsmodells (siehe Kap. 5.2.4) eine Verbesserung der Kunden- und Bürgerorientierung vor allem auf der intraorganisatorischen Ebene, d.h. auf der Ebene der einzelnen Behörden und Ämter. Die Kritik der Bürgerinnen und Bürger ist hier, wie erwähnt, uneindeutig und widersprüchlich, sie richtet sich sowohl gegen Phänomene der "Überbürokratisierung“ (Überregelung, Anonymität, Inflexibilität), wie auch der „Unterbürokratisierung“ (Willkür, Korruption; vgl. Grunow/Strüngmann 2008). Dagegen stellt die interorganisatorische Teildimension, bei der es um die Koordination und Zusammenarbeit zwischen unterschiedlichen Behörden, Verwaltungszweigen und -ebenen in Entscheidungsprozessen (insbesondere in Genehmigungs- und Bewilligungsverfahren) geht, offensichtlich die „Problemzone“ der Bürokratieproblematik in Deutschland dar (ausführlich Jann/Wegrich/Tiessen 2007).

Entbürokratisierung und Bessere Rechtsetzung sind generell beliebt - niemand ist gegen die Abschaffung unnötiger Bürokratie oder gegen bessere Gesetze - aber sie stoßen im Detail immer wieder auf erhebliche Widerstände, denn sie berühren die Interessen sektoraler Policy-Netzwerke. Diese sind im Konfliktfall meist durchsetzungsfähiger als die Promotoren der Entbürokratisierung, d.h. prinzipiell sind ,Generalisten', die sich für verwaltungspolitische Reformen wie Bürokratieabbau einsetzen, dem Netzwerk der ,Spezialisten' der betroffenen Politikbereiche in Wirtschaft, Gesellschaft, Politik und Verwaltung unterlegen (das sog Generalisten-Spezialisten-Dilemma, Jann/Wegrich 2008, dies. 2019).

Vor allem ist es aber auch eine gefährliche technokratische Illusion, dass sich Bürokratie durch die richtigen wissenschaftlichen Methoden, also etwa ,regulatory impact analysis' (RIA) einfach und konfliktfrei (also , unpolitisch') reduzieren lässt:

„Entbürokratisierung ist immer auch eine politische Frage und hängt somit von den Akteurs- und Interessenkonstellationen ab. Entbürokratisierung ist (wie Bessere Rechtsetzung) Querschnittspolitik und als solche vor allem dann wirkungsvoll durchzusetzen, wenn politische oder administrative Unterstützung gegenüber den im Zweifelsfall mächtigeren Sektor-/Ressortpolitiken mobilisiert werden kann" (Jantz/Veit 2019, S. 519). 
Dies gelingt nur durch die institutionelle und organisatorische Stärkung dieser generellen Anliegen und ihre Verankerung in den normalen bürokratischen Entscheidungs- und Koordinationsprozessen, wie dies in Deutschland durch die Etablierung des Normenkontrollrates durchaus in Ansätzen gelungen ist.

\subsubsection{Renaissance der Bürokratie?}

Trotz der vielfältigen und wie gezeigt seit langer Zeit etablierten Bürokratiekritik und den kontinuierlichen Forderungen nach Bürokratieabbau, ist es in der Verwaltungswissenschaft weitgehend Konsens, dass bürokratische Organisationen unverzichtbar sind, und es gibt sogar Stimmen, die eine Renaissance der Bürokratie sowohl empirisch konstatieren wie auch normativ fordern. Insbesondere in der neo-institutionalistischen, sog. ,skandinavischen Schule“ der Verwaltungswissenschaft (vgl. zum Folgenden Jann 2006a, S. 140ff.) argumentiert z. B. deren profiliertester Vertreter Johan P. Olsen $(2006,2008)$ gegen die These, Bürokratien seien organisatorische Dinosaurier, deren langsamen aber unvermeidbaren Todeskampf wir gerade beobachten, also , an undesirable and non-viable form of administration developed in a legalistic and authoritarian society and now inevitably withering away because it is incompatible with complex, individualistic and dynamic societies" (Olsen 2006).

Empirisch wird dem Befund von der schwindenden Bedeutung bürokratischer und hierarchischer Organisationen für moderne Gesellschaften widersprochen. In den Worten von Herbert Simon:

\footnotetext{
"We are so accustomed to hearing our society described as a market economy, that we are often surprised to observe that, since the time of Adam Smith, markets have steadily declined, and business (and governmental) organizations have steadily grown as the principal coordinators of economic activity" (Simon 2000, S. 1).
}

Weltweit beobachten wir eine rule explosion, die mit dem Schlagwort freer markets, more rules beschrieben wird (Vogel 2018). Insbesondere die Entwicklung der EU gilt als Hinwendung zu einem regulatory state, und die Ablösung klassischen Vertrauens in Bürokraten, Sozialarbeiter oder im Gesundheitsbereich, hat im Rahmen von NPM-Reformen gerade nicht zu weniger Regeln und weniger Kontrolle geführt, sondern zu einer audit explosion, und zum sog. auditory state (Power 2005). Regulierung und ihre Umsetzung ist daher seit einiger Zeit ein Schwerpunkt moderner sozialwissenschaftlicher Forschung über den Staat (Döhler/Wegrich 2010, siehe auch die Zeitschrift Regulation \& Governance).

Normativ, so die Argumentation, unterstützen und stärken bürokratisch strukturierte Organisationen wichtige Werte, Prinzipien und Ziele, also Vorstellungen 
von richtigem und angemessenem Verhalten, z. B. in Bezug auf Gleichbehandlung, Fairness, Verantwortlichkeit und Berechenbarkeit. Grundlage ist eine klassische institutionalistische Argumentation:

\footnotetext{
"Institutions give order to social relations, reduce flexibility and variability in behaviour, and restrict the possibilities of a one-sided pursuit of self-interest or drives. The basic logic of action is rule following - prescriptions based on a logic of appropriateness" (March/Olsen 2006, S. 8).
}

Bürokratien sind daher Ausdruck und Verstärker kultureller Werte, z. B. prozeduraler Rationalität und Verlässlichkeit, Rechtsstaatlichkeit und professioneller Standards. Identitäten, Werte und Normen von Akteuren sind aus dieser Sicht nicht Voraussetzung, sondern Ergebnis von institutionellen Arrangements. Im Prinzip werden Individuen also durch die Institutionen, in denen sie agieren, zu egoistischen und opportunistischen Nutzenmaximierern, zu kooperierenden, tauschorientierten Netzwerkern oder zu regelorientierten, neutralen und integren Amtsinhabern, eben Bürokraten.

Olsen argumentiert weiter, dass Bürokraten daher nicht in erster Linie Diener und Hüter der Herrschenden sind, sondern vor allem gesetzlicher und professioneller Regeln und der verfassungsmäßigen Ordnung. In Bürokratien gibt es daher mindestens drei konkurrierende Prinzipien mit unterschiedlichen Aufpassern und gate-keepern, nämlich Hierarchie (Politiker), Regeln (Gerichte) und Expertise (Professionen). Bürokratien zeichnen sich dadurch aus, dass sie in der Lage sind, zwischen diesen konkurrierenden Prinzipien zu vermitteln (Olsen 2008).

In der verwaltungswissenschaftlichen Diskussion spielt eine mögliche „Renaissance der Bürokratie" vor allem im Konzept des neo-weberianischen Staates eine Rolle (Bouckaert 2006, zusammenfassend Byrkefklot et.al. 2018, siehe auch unten 5.2.4). Empirisch wie normativ wird argumentiert, dass moderne Verwaltungen sowohl klassisch weberianische wie moderne managerialistische Merkmale aufweisen. Als zentrale weberianische Merkmale werden genannt:

- Bewahrung von Staat als zentralem Akteur gesellschaftsorientierter Problemlösung, Souveränität, Legitimation und Gemeinwohlorientierung;

- Bewahrung von Gleichheit vor dem Gesetz, Schutz vor willkürlichen und unvorhersehbaren Handlungen staatlicher Stellen, umfangreiche staatliche Rechenschaftspflicht;

- Bewahrung des öffentlichen Diensts mit eigenem Status, eigener Kultur und eigenen Beschäftigungsbedingungen;

- Bewahrung der zentralen Bedeutung des öffentlichen Rechts. 
„Neo“ an diesen modernen Verwaltungen soll u. a. sein:

- die Stärkung externer Orientierung an Kunden und Klienten;

- die Kombination repräsentativer Prozesse mit direkter Bürgerbeteiligung;

- die Stärkung von ex-ante Leistungssteuerung gegenüber prozeduraler ex-post Kontrolle;

- die Stärkung der Professionalisierung als Manager und

- die Stärkung von Effektivität, Nutzenmaximierung und Effizienz.

Schließlich spielt Bürokratie auch eine zentrale Rolle in den aktuellen Diskussionen über Good Governance (vgl. Hill 2005) oder Quality of Government (siehe insbesondere das Quality of Government Institute, https:/qog.pol.gu.se/). Dabei wird immer wieder zentral auf die Bedeutung von Rechtsstaatlichkeit (rule of law), Rechenschaftspflicht (accountability) und vor allem Unparteilichkeit (impartiality) als Grundlagen staatlichen Handelns und politischer Legitimation hingewiesen (als Übersicht Rothstein/Teorell 2008). In einer umfangreichen international vergleichenden Studie zeigen z. B. Dahlberg/Holmberg (2014), dass unparteiische und effektive Bürokratien für die Zufriedenheit der Bürgerinnen und Bürger weltweit wichtiger sind als die Ausprägungen repräsentativer Demokratie.

\subsection{Bürokratie in Deutschland: Strukturen der Aufbauorganisation}

Im Folgenden soll es darum gehen zu skizzieren, inwieweit öffentliche Organisationen in Deutschland tatsächlich dem Idealtypus einer bürokratischen Organisation entsprechen und wo sie ggf. warum von diesem abweichen. Dazu werden die beiden klassischen Bereiche Ministerien und Kommunalverwaltung betrachtet. Die dabei identifizierten Merkmale bürokratischer Organisationen gelten dabei auch, und oft in noch stärkerem Ausmaß, für nachgeordnete Behörden und Ämter.

\subsubsection{Organisation der Ministerien}

\subsubsection{Aufbau- und Ablauforganisation}

Ministerien unterstützen einerseits die Regierungstätigkeit, indem sie politische Entscheidungen des Ministers oder der Ministerin, insbesondere Gesetzgebung und Budgetentscheidungen vorbereiten, andererseits sind sie als oberste Bundes- oder 
Landesbehörden auch an der Durchführung der Gesetze, der Implementation von Politik beteiligt, in ihnen wird also regiert und verwaltet. Während Landesministerien vor allem in Ländern ohne Mittelinstanz auch vielfältige Vollzugsaufgaben wahrnehmen, bedienen sich die Bundesministerien im Wesentlichen der nachgeordneten Bundesbehörden - wenn der Bund überhaupt in die Implementation seiner Gesetze eingebunden ist (siehe Kap. 3.2). Zu den Aufgaben der Ministerien gehören neben diesen Vollzugsaufgaben, also der Steuerung und Überwachung nachgeordneter Behörden und des Gesetzes- und Haushaltsvollzugs, auch eine Reihe für die Politik wichtiger Hilfsaufgaben wie die Beantwortung parlamentarischer Anfragen, die Vorbereitung von Reden und generell die Aufbereitung und Zusammenstellung relevanter Informationen. Zentrale Aufgabe der Ministerien ist aber die Programmentwicklung, also der Entwurf und, vor allem, die Novellierung von Gesetzen, die Entwicklung von Rechtsverordnungen und Verwaltungsvorschriften, die Aufstellung des jeweiligen Haushalts und generell die Entwicklung und Planung neuer Politikinhalte und Policies.

Im Prinzip sind Ministerien in Deutschland im Wesentlichen einheitlich und nach bürokratischen Merkmalen aufgebaut. Im Folgenden wird dies anhand der Bundesministerien erläutert; Landesministerien weisen die gleichen Merkmale auf, sind allerdings kleiner und verfügen i.d.R. z.B. nicht über parlamentarische Staatssekretäre:

- An der Spitze des Ministeriums steht der politisch ernannte Minister oder die Ministerin. Diese sind keine Beamten, nach dem Grundgesetz dürfen sie auch keine weitere berufliche Tätigkeit ausüben.

- Jedem Minister sind (auf Bundesebene) i.d. R. ein oder zwei parlamentarische Staatssekretäre zugeordnet, die den Minister bei der Erfüllung der Regierungsaufgaben insbesondere hinsichtlich der Verbindung zum Bundestag und Bundesrat unterstützen und hier auch vertreten. Auch die parlamentarischen Staatssekretäre sind keine Beamten, sondern es handelt sich, wie der Name schon sagt, um Mitglieder des Parlaments. Mit dem Rücktritt des Ministers scheiden auch sie aus.

- An der Spitze jedes Ministeriums stehen ein oder mehrere beamtete Staatssekretäre, die den Minister nach innen in der Leitung der Ressorts unterstützen und als Behördenchefs die Dienstvorgesetzten der Mitarbeiter sind. Sie sind "politische Beamte“ (siehe 4.4.1)

- Die Ministerien gliedern sich in Abteilungen, Unterabteilungen und Referate. Diese werden von Ministerialdirektoren, Ministerialdirigenten sowie in den Referaten von Ministerialräten geleitet. 
- Als Stabseinrichtungen für die Leitung gibt es Ministerbüros mit persönlichen Referenten und Pressestellen, gelegentlich auch Stäbe oder Projektgruppen für besondere Aufgaben (etwa in der 13. Legislaturperiode im BMI die „Stabstelle Moderner Staat - Moderne Verwaltung").

Die zentralen Merkmale der Aufbau- und auch Ablauforganisation der Bundesministerien, der hierarchische Aufbau, die Art und Weise der Aufgabenerledigung und insbesondere auch die Formen der internen Koordination und der Kontakte nach außen sind in der GGO, der „Gemeinsamen Geschäftsordnung der Bundesministerien“ (BMI 2011) festgelegt, die es seit der Weimarer Republik gibt, und die zwar verschiedentlich vereinfacht und abgespeckt wurde, aber die klassische bürokratische Ablauforganisation nicht infrage stellt. Auf den ersten Blick weisen Ministerien damit alle Merkmale einer klassischen bürokratischen Organisation auf, insbesondere strikte Arbeitsteilung, hierarchische Kommunikation in einem Einliniensystem, Professionalisierung und klare Zurechenbarkeit und Verantwortlichkeit durch Aktenmäßigkeit und Schriftlichkeit. Die Basiseinheit der Ministerien, in denen sämtliche Aufgaben zunächst wahrgenommen werden, sind die Referate. Sie gelten daher als „Arbeitstiere“ der Ministerialverwaltung.

Die erste umfassende Untersuchung über die deutsche Ministerialverwaltung wurde Anfang der siebziger Jahre des letzten Jahrhunderts von der „Projektgruppe Regierungs- und Verwaltungsreform" vorgenommen (vgl. 5.2.1) ${ }^{61}$. Die vorherrschende Programmformulierung in den Ministerien wurde von Mayntz/Scharpf dabei als „reaktive Politik“ kritisiert, die vor allem heteronom, d. h. von äußeren Einflüssen und durch bürokratische Strukturen beeinflusst und daher in ihrer Reichweite und Zielsetzung als begrenzt, kurzfristig und umweltanpassend zu charakterisieren sei. Demgegenüber formulierten sie den Anspruch einer "aktiven Politik“, d.h. einer politik- und leitungsbestimmten Programmformulierung, die umfassende, längerfristige und umweltverändernde Politikinhalte unterstützen sollte. Gefordert wurde u. a. die Einrichtung von Planungsstäben und eine Reduzierung der Vielzahl von Referaten, insbesondere von sogenannten „Kleinstreferaten“ mit nur wenigen Mitarbeitern, weil eine übergroße Spezialisierung übergreifende Problemsichten eher erschwert und den Mechanismen der selektiven Perzeption und negativen Koordination Vorschub leistet. Von den von der Projektgruppe Regierungs- und Verwaltungsreform vorgeschlagenen Veränderungen der klassisch-bürokratischen Strukturen wurden nur wenige direkt umgesetzt (vgl. Müller 1977), aber dennoch

61 Die umfassendste Studie, die im Gefolge der Projektgruppe Regierungs- und Verwaltungsreform entstand, liegt leider nur auf Englisch vor, Mayntz/Scharpf 1975, siehe auf Deutsch vor allem dies.1973. 
hat sich die deutsche Ministerialbürokratie seit dieser Zeit verändert, insbesondere ist die Ministerialbürokratie „politischer" geworden (vgl. auch 4.4).

Insgesamt verfügen die Bundesministerien über gut 1000 Referate, ca. 240 Unterabteilungen und 110 Abteilungen. Diese Zahlen sind seit 1980 überraschend stabil, obwohl die Zahl der Ministerien in diesem Zeitraum von ca. 20 auf 13 reduziert wurde (siehe im Detail die international vergleichende Langzeitstudie zur Entwicklung der Strukturen der Ministerialverwaltung in fünf Ländern seit 1980, Fleischer et.al. 2018 und Bertels/Schulze-Gabrechten 2020).

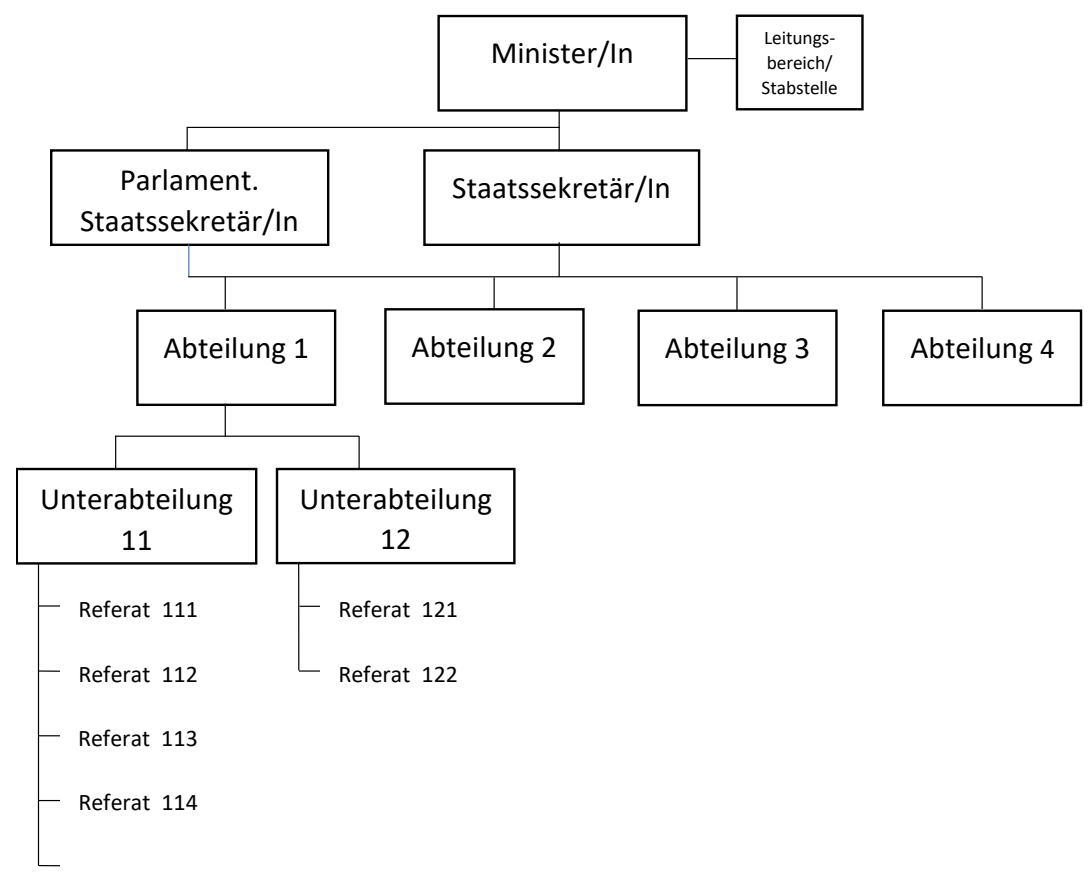

Abb. 47 Aufbau eines Ministeriums

Quelle: eigene Darstellung

$\mathrm{Zu}$ den gemeinsamen Organisationsvorgaben der GGO gehören neben der Definition der organisatorischen Kernelemente der Bundesministerien, also der Referate, Unterabteilungen und Abteilungen aber auch eine Reihe von „besonderen Organisationsformen“ (BMI 2011, \$10), z. B.: 
- Organisationseinheiten mit Stabsfunktion, vor allem zur Unterstützung der Leitung;

- Projektgruppen für „zeitlich befristete komplexe Aufgaben, die einen übergreifenden Personaleinsatz erfordern";

- Beauftragte der Bundesregierung, die Bundesbeauftragten sowie Koordinatorinnen und Koordinatoren der Bundesregierung.

Der Anteil dieser weiteren Einheiten nimmt im Zeitverlauf deutlich zu (vgl. im Detail Fleischer et.al. 2018, S. 32ff). Während sie 1980 lediglich ca. 7 \% der Einheiten in der Ministerialverwaltung auf Bundesebene ausmachen, liegt ihr Anteil 2015 bei $18 \%$. Insbesondere ab 1995 ist ein deutlicher Anstieg erkennbar. Vor allem der strukturelle Ausbau der Leitungsbereiche ist dabei bemerkenswert. Während Anfang der achtziger Jahre

„nur drei bis maximal sechs Bundesministerien derartige Einheiten mit organisatorischem Unterbau besaßen und die Mehrheit der Ministerien stattdessen nur singuläre Referate auf Leitungsebene organisierte, ist inzwischen in jedem Ressort eine solche Leitungseinheit mit untergeordneten Referaten eingerichtet und als Standardorganisationsformat etabliert" (Fleischer et.al. 2018, S.39, siehe im Detail zu dieser Entwicklung auch Hustedt 2013, S. 263f.).

Es geht bei diesen Einheiten vor allem um die direkte politische Beratung der Minister, aber auch um Kommunikation und Pressearbeit, Koordination und längerfristige Planung. Auch wenn sich die schon von der „Projektgruppe Regierungs- und Verwaltungsreform" vorgeschlagenen umfassenden Planungsstäbe nicht durchgesetzt haben, ist also die steigende Bedeutung von Stäben vor allem zur direkten Unterstützung des Ministers oder für begrenzte besondere Aufgaben unverkennbar. Dabei dürfen die durch Stäbe hervorgerufenen Konflikte in Organisationen nicht unterschätzt werden. Überspitzt gesagt sehen Stäbe Linienorganisationen als „Fachidioten“, die nicht in der Lage sind, ihre Aufgaben in einen größeren Zusammenhang einzuordnen, während wiederum die Fachleute in der Linie den Stäben vorwerfen, sie würden sich kontinuierlich besserwisserisch in Angelegenheiten einmischen, in denen sie über keinerlei Kompetenz verfügten.

Aus diesen Gründen sind auch andere Organisationsformen, etwa eine professionell-teamartige Organisation mit einer weniger starren Festlegung des Aufgabenund Verantwortungsbereichs einzelner Mitarbeiter und einer eher netzwerkartigen Kommunikations- und Kontrollstruktur, z. B. in Projektgruppen, in der klassischen deutschen Ministerialbürokratie immer noch selten. Das gleiche gilt für Formen der Matrix- oder Mehrlinienorganisation, in denen Einheiten gleichzeitig zweien oder sogar mehreren Einheiten untergeordnet werden. Solche Organisationsformen 
sind nicht ausgeschlossen, aber sie sind der klassischen bürokratischen Einlinienorganisation mit eindeutigen Kommunikations- und Kontrollstrukturen fremd. Insgesamt bleibt aber festzuhalten, dass es zunehmend mehr strukturelle Flexibilität in der deutschen Ministerialverwaltung gibt, als normalerweise angenommen wird, und die Vielfalt dieser Organisationseinheiten beständig wächst.

\subsubsection{Bonn-Berlin}

Eine Besonderheit der Ministerialverwaltung des Bundes ist schließlich der doppelte Regierungssitz in Berlin und Bonn (vgl. Jann/Wewer 1998; Hustedt 2015 m. w. A.). Dieser ist eine Folge des Beschlusses des deutschen Bundestages vom Juni 1991 für Berlin als Bundeshauptstadt ${ }^{62}$ und für Bonn als Bundesstadt. Die wichtigsten Entscheidungen in diesem Zusammenhang, nämlich die Festlegung des sog. Kombinationsmodells mit Bundesministerien sowohl in Berlin wie in Bonn und der Beschluss, welche Bundesministerien mittelfristig nach Berlin gehen und welche in Bonn verbleiben sollten, wurden bereits im gleichen Jahr im Bundeskabinett gefasst und waren durch politische Kompromisse mit der beteiligten Region (Bonn, NRW) und den Beschäftigten des Bundes geprägt.

Allerdings dauerte es bis zum April 1994, bis diese Eckpunkte im sog. Berlin/ Bonn-Gesetz festgeschrieben wurden. Dazu gehören die Sicherstellung einer „dauerhaften und fairen Arbeitsteilung zwischen der Bundeshauptstadt Berlin und der Bundesstadt Bonn“, die Ansiedlung des „Kernbereichs der Regierungsfunktionen“ in Berlin, „Erhalt und Förderung politischer Funktionen“ in Bonn in den Politikbereichen Bildung und Wissenschaft, Forschung und Technologie, Telekommunikation, Umwelt und Gesundheit, Ernährung, Landwirtschaft und Forsten, Entwicklungspolitik sowie Verteidigung, Kompensation für Bonn und für die Mitarbeiter in Bonn, Bundesministerien in beiden Städten, Dienstsitze aller Ministerien in beiden Städten, Bewahrung des „größten Teils der Arbeitsplätze der Bundesministerien in der Bundesstadt Bonn".

Bereits 1992 hatte das Bundeskabinett beschlossen und dem Bundestag mitgeteilt, dass etwa zwei Drittel der Arbeitsplätze der Ministerien in Bonn verbleiben sollten. Gleichzeitig sollten alle Ministerien Dienstsitze in beiden Städten unterhalten, wobei die Berliner Dienstsitze der Bonn-Ministerien etwa $10 \%$ des Personalbestandes der Ministerien umfassen sollten. Diese Festschreibungen des Regierungsumzuges, insbesondere das sog. „Kombinationsmodell“, wurden von Beginn an kritisiert, weil es u.a. zu einer erschwerten Koordination und Kommunikation zwischen

62 Seit dem 1.9.2006 ist die Hauptstadt Berlin im GG in Art. 22 erwähnt, woraus eine finanzielle Mitverantwortung des Bundes für alles, was mit der Hauptstadtfunktion verbunden ist, erwächst. 
und sogar innerhalb der Ministerien, zu suboptimaler Kommunikation zwischen Parlament und Regierung, zu einer Duplikation von Funktionen in Berlin und Bonn, zu umfangreicher, nicht notwendiger Reisetätigkeit, zu größerer Distanz zwischen politischer Führung und Mitarbeitern, zu einer Zweiklassengesellschaft wichtigerer (Berlin-) und unwichtigerer (Bonn-)Ministerien und schließlich auf längere Sicht zur „Rutschbahn“ sämtlicher Ministerien von Bonn nach Berlin führen werde (vgl. Jann 1997).

Nachdem der Regierungsumzug im Sommer 1999 durchgeführt wurde ist es tatsächlich zu einer schnellen Verlagerung aller wichtigen Regierungsfunktionen nach Berlin gekommen (ausführlich Hustedt 2015). Von Beginn an haben Ressortchefs der sog. Bonn-Ministerien erklärt, dass die vorgesehenen $10 \%$ Beschäftigte in Berlin nicht ausreichen und haben begonnen, wichtige Teile der Ministerien nach Berlin zu verlagern. Derzeit haben noch sechs Ministerien ihren ersten Dienstsitz in Bonn (Bundesministerium der Verteidigung (BMVg), Bundesministerium für Gesundheit (BMG), Bundesministerium für Umwelt, Naturschutz und nukleare Sicherheit (BMU), Bundesministerium für Ernährung und Landwirtschaft (BMEL), Bundesministerium für Bildung und Forschung (BMBF) und Bundesministerium für wirtschaftliche Zusammenarbeit und Entwicklung (BMZ)), dabei befinden sich jeweils noch etwa $80 \%$ der Planstellen dieser Ministerien in Bonn (Verteidigung $90 \%$ ). Insgesamt waren am Stichtag 31.12.2015, für den die letzten offiziellen Zahlen vorliegen, 12.654 (64,3\%) Mitarbeiter/-innen in Berlin und 7.030 (35,7\%) in Bonn beschäftigt. Damit ist die Festlegung des Gesetzes, dass „insgesamt der größte Teil der Arbeitsplätze der Bundesministerien in der Bundesstadt Bonn erhalten bleibt" ( $\$ 4$, Abs. 4) schon lange obsolet. Selbst der offizielle Bericht der „Beauftragten der Bundesregierung für den Berlin-Umzug“ (BMUB 2017, S.4f.) stellt fest: „In der Vergangenheit wurden aus verschiedenen Gründen zunehmend Ministeriumsarbeitsplätze von Bonn nach Berlin verlagert“, und dass „die Arbeitsteilung sowohl den fachlichen als auch den persönlichen Austausch innerhalb der jeweiligen Ressorts erschwert". Insgesamt gibt es daher regelmäßig Forderungen, diese Doppelstrukturen aufzulösen und sämtliche Ministerien nach Berlin zu verlagern, die aber bisher aus Rücksicht auf die Region Bonn nicht aufgegriffen wurden - obwohl es im Jahr 2015 ca. 37.300 Arbeitsplätze in Einrichtungen des Bundes in der Region Bonn gab, gegenüber ca. $35.100 \mathrm{im}$ Jahr 2020, und Bonn im Jahresdurchschnitt 2014 mit 99.492 Euro je Erwerbstätigen das höchste BIP in NRW hatte (ebd.). 


\subsubsection{Organisation der Kommunalverwaltung}

Kreise und Gemeinden verfügen im Rahmen der landesrechtlichen Bestimmungen über die Organisationshoheit in ihrem Gebiet, d.h. sie verfügen über das Recht auf eigenverantwortliche Gestaltung ihrer internen Organisation. Dies umfasst sowohl die Wahl der Organe, die Organisation der gemeindlichen eigenen Verwaltung und die Regelung der ,inneren Verfassung ““ der Gemeinde durch Erlass der Hauptsatzung und der Geschäftsordnung. Die Organisationsgewalt über die gemeindliche Verwaltung gilt sowohl für Selbstverwaltungsaufgaben als auch für Auftragsangelegenheiten.

Der direkt gewählte hauptamtliche Bürgermeister bzw. der Landrat ist als Verwaltungschef verantwortlich für die Leitung und Verteilung des Geschäftsgangs der gesamten Verwaltung und er bereitet die Beschlüsse der Gemeindevertretung, der Bezirksvertretungen und der Ausschüsse vor. Er ist Dienstvorgesetzter der Wahlbeamten, Beamten, Angestellten und Arbeiter. ${ }^{63}$

Der Bürgermeister bzw. Landrat ist kommunaler Wahlbeamter ${ }^{64}$. In allen Kreisverwaltungen und ab einer bestimmten Größenordnung in den Städten und Gemeinden gibt es hauptamtliche Beigeordnete (ansonsten ehrenamtliche). Diese werden von der Gemeindevertretung gewählt, sind ebenfalls kommunale Wahlbeamte auf Zeit (meistens acht Jahre) und für die politische Leitung eines Geschäftsbereichs zuständig. Bemerkenswert ist, dass sie i.d.R. nicht nach dem Prinzip „Mehrheit vs. Minderheit“" gewählt werden, sondern dass alle wichtigen Parteien und anderen Gruppen in den Gemeinderäten beteiligt werden und so, je nach ihrer Stärke, in der politischen Führung der lokalen Verwaltungen vertreten sind. In einigen Bundesländern ist diese Proporzwahl sogar in den gesetzlichen Regelungen für die Kommunalverwaltung vorgeschrieben (z. B. in Baden-Württemberg und Sachsen), aber selbst, wenn nicht vorgeschrieben, ist diese Praxis weit verbreitet (vgl. Schleer 2014). Obwohl die Beigeordneten von der Gemeindevertretung gewählt sind, besitzt der Bürgermeister allerdings ein Weisungsrecht gegenüber den Beigeordneten.

Der Bürgermeister verfügt über das Organisationsrecht, in das die Gemeindevertretung nur in gesetzlich geregelten Ausnahmefällen eingreifen darf. Gleiches gilt auch in den meisten Bundesländern für die Regelung der Geschäftsbereiche

63 Disziplinarvorgesetzte ist allerdings die Aufsichtsbehörde.

64 Gegenüber dem früheren ehrenamtlichen Bürgermeister, der (in NRW) in Großstädten eine Aufwandsentschädigung von ca. 30.000 Euro erhielt, verfügt der hauptamtliche Bürgermeister nun über ein jährliches Einkommen zwischen 90.000 und 170.000 Euro je nach Gemeindegröße (B2 bis B11). Allerdings liegt zum Vergleich dazu das Einkommen des Vorstandssprechers von städtischen Versorgungsunternehmen in Großstädten mitunter bei 300.000 Euro. 


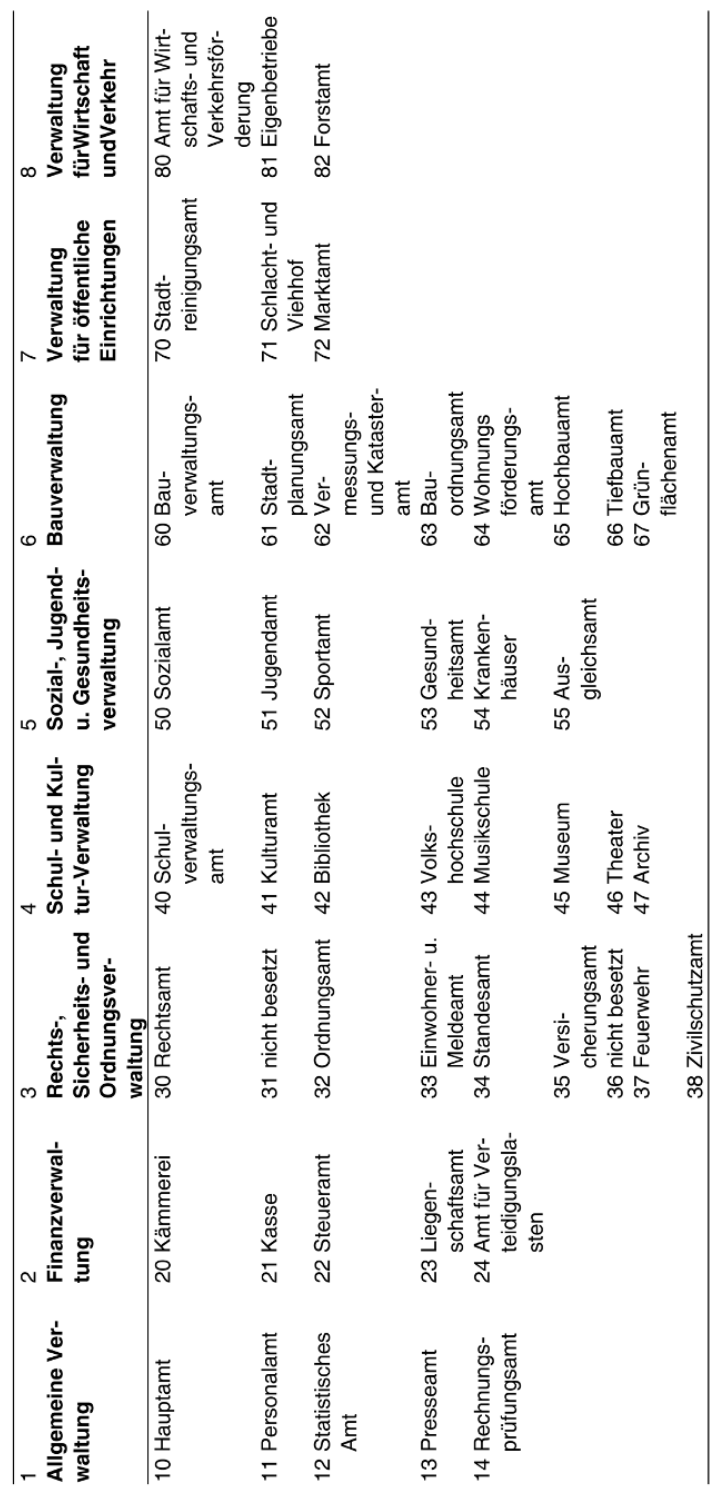

Abb. 48 Verwaltungsgliederungsplan der KGST ${ }^{65}$

65 In kleineren Kommunen gibt es nicht so starke Ausdifferenzierungen. 
der Beigeordneten. Der Bürgermeister kann selbstständig einen Geschäfts- und Organisationsverteilungsplan erlassen und durch Einzelanweisungen die Geschäfte auf die Verwaltungsmitarbeiter verteilen. Zudem verfügt er über eine Beanstandungspflicht, wenn er der Meinung ist, dass gegen geltendes Recht verstoßen wird. Hier gibt es eine aufschiebende Wirkung und die Aufsichtsbehörde entscheidet. Der hauptamtliche Bürgermeister hat weiterhin ein Widerspruchsrecht mit aufschiebender Wirkung gegenüber Gemeinderatsbeschlüssen, die, seiner Meinung nach, das Wohl der Gemeinde gefährden. Dieser Beschluss muss dann noch einmal im Rat behandelt werden. Ein weiterer Widerspruch ist unzulässig.

Auch bei der Darstellung der inneren Organisation der Gemeinden kann zwischen der institutionellen Organisation, das heißt dem Aufbau der Verwaltung, und der funktionellen Organisation, das heißt dem Ablauf des Verwaltungshandelns, unterschieden werden. Die Aufbauorganisation in Kommunalverwaltungen orientierte sich jahrzehntelang weitgehend einheitlich in Gemeinden aller Größenklassen und Ländern an dem schon in den fünfziger Jahren entwickelten, aber mehrfach neueren Entwicklungen angepassten Verwaltungsgliederungsplan der Kommunalen Gemeinschaftsstelle für Verwaltungsmanagement (KGSt).

Wie unschwer zu erkennen, ist auch die Kommunalverwaltung hochgradig arbeitsteilig organisiert und stark hierarchisiert. Die zentrale organisatorische Gliederungsgröße ist das Amt. Die Ämter sind die den Vollzug der kommunalen Aufgaben tragenden Organisationseinheiten, die nach außen hin selbstständig in Erscheinung treten. Die Amtsleiter haben die Fach- und Dienstaufsicht gegenüber ihren Mitarbeitern und verfügen damit über erhebliche Machtpotenziale. Der Gliederungsplan ordnet die Ämter acht Aufgabenhauptgruppen zu. Diese unter fachlichen Gesichtspunkten gebildete Systematik bildet die Basis für den organisatorischen Aufbau der Verwaltung. Unter diesen Hauptaufgabengruppen werden die Ämter der Verwaltung nach Zuständigkeit aufgeteilt, wobei ihnen zweistellige arabische Zahlen zugewiesen werden. Die Ämter sind dann noch weiter aufgegliedert in Abteilungen (dreistellig) und Sachgebiete (vierstellig). Insgesamt sind folgende Arbeitseinheiten von unten nach oben zu unterscheiden: Stelle, Sachgebiet, Abteilung, Amt, Dezernat.

Eine Ausnahme von der fachlichen Gliederung bilden die Querschnittsämter, deren Aufgaben darin bestehen, das Funktionieren der Verwaltung sicherzustellen. Die wichtigsten Querschnittsämter sind das Hauptamt, das Personalamt und die Kämmerei. Einzelne Abweichungen von diesem Gliederungsplan sind in der Regel ortsbedingten Umständen geschuldet, insbesondere in den kreisfreien Städten sind die Abweichungen jedoch marginal. Die Zahl der Ämter hängt vor allem von der Größe der Stadtverwaltung ab. Der Gliederungsplan sagt aber noch nichts über die 
politischen und administrativen Zuständigkeiten innerhalb der Verwaltung aus. Hier ist in der Regel der Dezernatsverteilungsplan aufschlussreicher.

Dieser ordnet einzelne Verwaltungsbereiche den jeweils zuständigen Beigeordneten zu. Dezernate sind also Geschäftsbereiche der Beigeordneten, die von der Gemeindevertretung für eine bestimmte Zeit (z. B. acht Jahre) gewählt sind. Über die Zahl der Beigeordneten und den Dezernatsverteilungsplan entscheidet ebenfalls die Gemeindevertretung innerhalb des von der Gemeindeordnung vorgegebenen Rahmens. Der Zuschnitt und die Anzahl der Dezernate sind deshalb sowohl politisch motiviert wie an Kenntnissen der Dezernenten orientiert. Die Dezernenten sind die direkten Vorgesetzten der Amtsleiter.

In den letzten 25 Jahren hat es in einigen Kommunen Veränderungen im Zuge der Verwaltungsreformen nach dem New Public Management-Modell gegeben, so dass man nicht mehr flächendeckend von der Gültigkeit des KGSt-Organisationsmodells und der eben dargestellten Hierarchieebenen ausgehen kann. Vor allem in vielen Mittel- und Großstädten sind im Zuge des Neuen Steuerungsmodells (NSM) neue Organisationsformen geschaffen worden. So wird in einer repräsentativen Umfrage unter den deutschen Kommunen über 10.000 Einwohner aus dem Jahr 2005 (vgl. Bogumil/Grohs u. a. 2007 sowie Kapitel 5.2.3) im Bereich der Umgestaltung von Organisationsstrukturen die Einführung von Fachbereichsstrukturen mit einem Anteil von fast $44 \%$ als der Modernisierungsschritt genannt, der umgesetzt wurde. ${ }^{66}$ Seit dieser Zeit gibt es jedoch keine weiteren repräsentativen Erkenntnisse, in welchem genauen Ausmaß es zu Veränderungen der Aufbauorganisation gekommen ist. Ein neues einheitliches Aufbaumodell ist ebenso nicht erkennbar.

Die funktionelle Organisation, mithin der Ablauf des Verwaltungshandelns, regeln die zentrale Geschäftsordnung und allgemeine Geschäftsanweisungen. Die Regelungen beziehen sich auf Fragen der Zusammenarbeit zwischen den Dienststellen, den Verkehr mit der Bevölkerung und die Organisation des Geschäftsganges (Post, Eingangsbearbeitung, Schriftverkehr, Zeichnungsbefugnis, Aktenführung. Neben der Aufgabenwahrnehmung in der "Kernverwaltung" werden kommunale Aufgaben auch in anderen Organisationsformen vorgenommen, also in Regiebetrieben, kommunalen Eigenbetrieben und verschiedenen privatrechtlichen Formen. Zudem gibt es als Organisationsform für interkommunale Zusammenarbeit die Zweckverbände oder höheren Kommunalverbände.

66 Beispielhaft kann dieses neue Vorgehen an der Stadt Arnsberg dargestellt werden. Hier wurden aus vier Dezernaten, 22 Ämtern und 64 Abteilungen im neuen Organisationsplan sieben Fachbereiche mit 32 Untereinheiten, also aus 90 Einheiten wurden somit knapp 40 (vgl. ausführlich Bogumil/Holtkamp 2013, S. 72ff.). Dadurch sollen die enormen Abstimmungsnotwendigkeiten in einer sehr spezialisierten Verwaltung vermieden werden. 
Zusammenfassend ist trotz aller Reformen der letzten Jahre davon auszugehen, dass sowohl im Bereich der Ministerial- wie auch abgeschwächt der Kommunalverwaltung noch immer die klassischen bürokratischen Merkmale der weitgehenden Arbeitsteilung, der hierarchischen Kommunikation und Kontrolle sowie der Aktenmäßigkeit und Professionalität gelten und dass dies auch für den weitaus größten Teil der diesen Behörden nachgeordneten Organisationen gilt.

\subsubsection{Begriffe der Aufbauorganisation}

Abschließend soll kurz noch einmal die Begrifflichkeit der unterschiedlichen Aufbauorganisationen zusammengefasst werden, denn entgegen der Vorstellung, in der juristisch geprägten Verwaltung seien Begriffe eindeutig besetzt, herrscht einige Verwirrung in der deutschen Verwaltungssprache. "Abteilung" ist die oberste Organisationsebene in Ministerien, aber eine eher untergeordnete Ebene in der Kommunalverwaltung. „Dezernate“ in Bezirksregierungen entsprechen Referaten auf Ministerialebene. Vor allem aber die Bezeichnung „Amt“ verfügt in der Verwaltung über noch viel mehr sehr unterschiedliche Bedeutungen (das Verwaltungslexikon Eichhorn u. a. 2003 enthält auf über sechs Seiten beinahe 50 Stichworte zu diesem Thema). Z.B. ist ein Amt

- nach dem Dezernat die oberste Organisationsebene in der Kommunalverwaltung (z.B. Einwohnermeldeamt, Rechtsamt),

- eine Verwaltungsgemeinschaft von Gemeinden (z. B. in Schleswig-Holstein und Brandenburg),

- im Dienstrecht die Bezeichnung der Übertragung einer funktionsgebundenen Aufgabe an eine Person, die dann ein Amt innehat und eine Amtsbezeichnung führen darf (z. B. Präsident des Umweltbundesamtes, Staatssekretär, Abteilungsleiter, Professor), und schließlich

- die Bezeichnung bestimmter staatlicher Behörden (sowohl auf der oberen Ebene, etwa Umweltbundesamt, Bundeskriminalamt, Bundesamt für Migration und Flüchtlinge, wie auf der unteren Ebene, Amt für Agrarordnung, Gesundheitsamt oder Finanzamt).

Wie diese verschiedenen Bezeichnungen systematisch vergleichbar sind, verdeutlicht die folgende Abbildung: 


\begin{tabular}{lll}
\hline & $\begin{array}{l}\text { Ministerium } \\
\text { (Bund und Land) }\end{array}$ & $\begin{array}{l}\text { Kommune } \\
\text { (Gemeinde und Kreis) }\end{array}$ \\
\hline $\begin{array}{l}\text { oberste politische } \\
\text { Leitung }\end{array}$ & $\begin{array}{l}\text { Regierungschef (Bundes- } \\
\text { kanzler, Ministerpräsident) }\end{array}$ & $\begin{array}{l}\text { Verwaltungschef (Bürger- } \\
\text { meister, Landrat) }\end{array}$ \\
\hline $\begin{array}{l}\text { politische Leitung des } \\
\text { einzelnen Ressorts }\end{array}$ & $\begin{array}{l}\text { Minister } \\
\text { (kein Beamter) }\end{array}$ & $\begin{array}{l}\text { Beigeordneter, Dezernent } \\
\text { (Wahlbeamte) }\end{array}$ \\
\hline $\begin{array}{l}\text { administrative } \\
\text { Leitung }\end{array}$ & $\begin{array}{l}\text { (beamteter) } \\
\text { Staatssekretär }\end{array}$ & $\begin{array}{l}\text { Beigeordneter, Dezernent } \\
\text { (Wahlbeamte) }\end{array}$ \\
$\begin{array}{l}\text { horizontale Differenzie- } \\
\text { rung nach }\end{array}$ & Ressort & Dezernat \\
$\begin{array}{l}\text { vertikale Differenzierung } \\
\text { nach }\end{array}$ & $\begin{array}{l}\text { - Abteilung } \\
\text { - Unterabteilung } \\
\text { - Referat }\end{array}$ & $\begin{array}{l}\text { - Amt } \\
\text { Abteilung }\end{array}$ \\
\hline
\end{tabular}

Abb. 49 Aufbauorganisation in Ministerien und Kommunen Quelle: eigene Darstellung

\subsection{Verfahren: Entscheidungen in der Verwaltung}

Wenn man die Bedeutung interner Strukturen und Prozesse für das Handeln der Verwaltung analysieren und verstehen will, ist es hilfreich, mit Entscheidungsprozessen zu beginnen. Tatsächlich produzieren Verwaltungen vor allem Entscheidungen:

\footnotetext{
„In einem sehr allgemeinen Sinne können Verwaltungen begriffen werden als soziale Systeme, die bindende Entscheidungen produzieren, und öffentliche Verwaltungen als solche, deren Entscheidungen gesamtgesellschaftlich verbindliche Wirkungen haben“" (Luhmann 1971, S. 165).
}

Zur Darstellung bietet sich wiederum eine Orientierung an dem einfachen Phasen- oder Zyklenmodell (Policy Cycle) der Politikfeld- oder Policy-Forschung an, das die Phasen der Politikformulierung (policy formation), Politikumsetzung oder -durchführung (policy implementation) sowie Bewertung (evaluation) und ggf. Beendigung (termination) unterscheidet (vgl. Kapitel 2.1; Jann/Wegrich 2014). Ein zentrales Ergebnis der empirischen Verwaltungsforschung besteht darin, gezeigt zu haben, dass öffentliche Verwaltungen oder allgemeiner öffentliche Organisationen, in allen diesen Phasen eine entscheidende Rolle spielen: 
- im Bereich der Politikformulierung z.B. bei der Vorbereitung von Gesetzen, Regierungsprogrammen, Plänen und im Budgetprozess, aber auch schon in der Phase des Agenda Settings und der Problemdefinition,

- während der Implementationsphase, da öffentliche Organisationen nur sehr unvollständig durch Gesetze gesteuert werden können (Grenzen der legislativen Programmsteuerung) und daher bei der Umsetzung über erhebliche Handlungsspielräume verfügen,

- und schließlich auch bei der Evaluation politischer Programme, weil es in erster Linie der Verwaltungsapparat ist, der in der Lage ist, entsprechende Informationen zu generieren und zu analysieren.

Politikformulierung und -durchführung können so als kontinuierlicher Entscheidungs- und Auswahlprozess verstanden werden, d.h. in und mit Verwaltungen wird entschieden über die Definition sozialer Wirklichkeit (was ist ein/das Problem?), über politische Ziele und Prioritäten, über Sinn und Zweck, Nutzen und Kosten alternativer Maßnahmen und Programme, über die Rechtsform politischer Programme, ihre Finanzierung, über Zweck- oder Zielprogramme, über jeden einzelnen Schritt der Implementation und schließlich auch über den Erfolg oder Misserfolg politischer Programme oder administrativer Maßnahmen. In jeder Phase des Policy-Cycle wird entschieden, dabei sind nicht einzelne Entscheidungen, der einzelne Auswahlakt das essenzielle Element, sondern der gesamte Entscheidungsprozess determiniert das Ergebnis, den Output.

Im Folgenden soll es darum gehen, einige empirische und theoretische Erkenntnisse der Verwaltungs- und Organisationswissenschaft über diese Prozesse kurz zusammenzufassen. Es soll gefragt werden, wie tatsächlich entschieden wird und welche Bedeutung verwaltungsinterne Strukturen und Prozesse für den Policy-Output des politisch-administrativen Systems haben, also für Politikinhalte und deren Umsetzung.

\subsubsection{Politikformulierung und Planung}

In der Phase des Agenda Settings, der Problemdefinition und der Politikformulierung geht es im Prinzip um Entscheidungen über zukünftige Aktivitäten: Welche Probleme sollen durch welche Maßnahmen, Instrumente und Programme zukünftig wie bearbeitet werden? Dies ist das klassische Problem der Planung. ${ }^{67}$

67 In der Frühphase der westdeutschen Verwaltungsforschung wurden daher Probleme der Politikformulierung und -umsetzung fast ausschließlich unter der Überschrift 
Planung hat umgangssprachlich und auch wissenschaftlich verschiedene Bedeutungen, aber im Kern geht es jeweils um einen Entwurf, der den Weg zu einem Ziel ebnet (nach lateinisch planum = eben). Kern ist also die Sicherung vor ungewissen Zukünften durch eine rationale, d.h. Zweck-Mittel-orientierte Zukunftsorientierung. Es geht um „vorausschauendes Setzen von Zielen und gedankliches Vorwegnehmen der ihrer Verwirklichung erforderlichen Verhaltensweisen", oder in der Diktion von Luhmann um „Entscheidungen über künftige Entscheidungen“. Nach einer anderen klassischen Definition von Frido Wagener ist Planung der Übergang vom Zufall zum Irrtum. Tatsächlich gehört Planung zum Standardrepertoire der öffentlichen Verwaltung in Deutschland.

\subsubsection{Planung in der Bundesrepublik Deutschland}

In einer groben Systematisierung kann man drei verbreitete öffentliche Planungsarten unterscheiden:

- In der Raumplanung geht es um Festlegungen der zukünftigen Nutzung des knappen Gutes „Raum“, also von Flächen oder Grundstücken, z. B. durch kommunale Bauleitplanung und Flächennutzungsplanung, aber auch durch regionale Raumordnungspläne oder etwa durch die Festlegung von Naturschutzgebieten.

- Bei der Finanzplanung geht es um Festlegungen, für welche öffentlichen Aktivitäten wann wie viel Geld bereitgestellt werden soll und kann. Das zentrale Instrument ist hier das jährliche Budget (auf allen Ebenen der Verwaltung und nicht zuletzt für jede einzelne öffentliche Organisation), aber es gibt zumindest auch Versuche längerfristiger Finanzplanungen, etwa "mifrifi“ (mittelfristige Finanzplanung).

- Daneben gibt es in fast allen Sektoren und Ressorts der Verwaltung Fachplanungen, also etwa Hochschulentwicklungspläne, Krankenhausbedarfspläne, Seniorenpläne, Frauenförderpläne.

Offensichtlich wäre es sinnvoll und hilfreich, wenn alle diese Pläne - im Sinne positiver Koordination - kontinuierlich aufeinander abgestimmt und miteinander koordiniert wären. Dies ist insbesondere in den sechziger und siebziger Jahren unter der Überschrift integrierte Entwicklungsplanung diskutiert und versucht worden. Die Erfahrungen mit diesem Instrument sind allerdings eher ernüchternd. Eine wirkliche integrierte Entwicklungsplanung, in der alle relevanten Raum-, Finanz- und Fachplanungen für einen längeren Zeitraum aufeinander abgestimmt werden, gibt es allenfalls für kleinere Entwicklungsgebiete. Ansonsten überfordert 
eine solche Planung sowohl die Informations- wie die Konfliktlösungskapazitäten unseres politisch-administrativen Systems (siehe oben unter 4.1.2 Koordination).

Eine zweite Systematisierung unterscheidet Planungsarten nach dem Grad ihrer Verbindlichkeit, also

- Aufgabenplanung, in der allgemeine Aufgaben einer Organisation oder eines Politikfeldes definiert werden (etwa das Regierungsprogramm zu Beginn einer Legislaturperiode);

- Programmplanung, bei der bestimmte umfangreiche staatliche Vorhaben festgelegt werden (etwa im Bundesverkehrswegeplan);

- Projektplanung, in der die Durchführung spezifischer Projekte programmiert wird (etwa die Trassierung einer Autobahn oder der Ausbau bestimmter Forschungsschwerpunkte), und schließlich

- Maßnahmenplanung, bei der eine einzelne Maßnahme im Detail „durchgeplant“ wird (etwa der Bau einer einzelnen Brücke oder die Festlegung eines bestimmten Schutzgebietes).

Im Prinzip kann man öffentliche Planungen also nach dem Grad der Konkretisierung von materiellen (Zielen), zeitlichen, räumlichen und finanziellen Festlegungen unterscheiden. Für die Verwaltungsforschung ist in diesem Zusammenhang relevant, wie diese Pläne und Entscheidungen zu Stande kommen und durch welche verwaltungsinternen und -externen Strukturen und Prozesse sie beeinflusst werden.

\subsubsection{Rationale Entscheidungstheorien und begrenzte Rationalität}

Die klassische Entscheidungstheorie hat ein klares normatives Bild von rationalen (Planungs-) Entscheidungen, die etwa nach folgendem Schema ablaufen sollten:

- Definition und Analyse der zu lösenden Probleme,

- Definition der zu erreichenden Ziele,

- Suche nach möglichen Alternativen der Zielerreichung (Mittel-Zweck-Analyse),

- Analyse der jeweiligen Kosten und Nutzen verschiedener Alternativen (einschließlich der wahrscheinlichen Effektivität der Problemlösung),

- vergleichende Analyse der Alternativen sowie

- Auswahl der besten Alternative zur Erreichung der festgelegten Ziele.

So, oder so ähnlich, sollte rational entschieden werden. Leider ist aber ein zentrales Ergebnis der modernen Organisationsforschung, dass dieses normative Modell fast immer unrealistisch ist. Von zentraler Bedeutung ist in diesem Zusammenhang das 
Konzept der „,begrenzten Rationalität“, der bounded rationality des Nobelpreisträgers Herbert A. Simon (vgl. hierzu ausführlich Bogumil/Schmid 2001, Kapitel 2).

Herbert A. Simon (1916-2001) war ursprünglich Politik- und Verwaltungswissenschaftler, der aber als Ökonom berühmt wurde. Er gilt als eigentlicher Begründer der verhaltenswissenschaftlichen Entscheidungstheorie. 1978 erhielt er den Nobelpreis für Wirtschaftswissenschaften für seine bahnbrechende Erforschung von Entscheidungsprozessen. In der Organisationstheorie wurde er berühmt durch seine Kritik des unrealistischen Nutzenmaximierungsmodells der Mikroökonomie und sein Modell der „bounded rationality“. Eines seiner Vorbilder war Barnard, der auch das Vorwort zu Simons Dissertation „Administrative Behavior“von 1945 schrieb. Ähnlich wie Barnard zielt Simon auf eine allgemeine Organisationstheorie, die sich primär auf Entscheidungen in Organisationen bezieht (administrative organization).

Für Simon war die anwendungsorientierte Erforschung von Verwaltungssystemen nur auf der Grundlage empirischer und verhaltensorientierter Analysen von Entscheidungsprozessen möglich. Ausgangspunkt seiner Auseinandersetzung mit Organisationsproblemen war die öffentliche Verwaltung, siehe das Vorwort seiner berühmten Dissertation „Administrative Behavior“: „Diese Studie soll Handwerkszeug für die Wissenschaft von der öffentlichen Verwaltung bereitstellen. Sie ist aus der Überzeugung entstanden, dass uns in diesem Bereich noch die angemessenen sprachlichen und begrifflichen Mittel fehlen, um Wesen und Bedeutung selbst einer einfachen Verwaltungsorganisation in einer Weise zu erfassen, die als Grundlage für die wissenschaftliche Beurteilung der Tauglichkeit ihrer Struktur und Arbeitsweise dienen kann" (Simon 1955, S. X).

Nach Simon beabsichtigen Akteure durchaus rational zu entscheiden und zu planen, können dieses Ziel aber nie erreichen, weil unser Wissen, unsere Ressourcen und unsere Kapazität, Informationen zu verarbeiten, begrenzt sind. Individuen und Organisationen haben daher Erkenntnisgrenzen, wenn es darum geht

- klare Ziele zu formulieren und zu ordnen, denn unser Wissen über die Bedingungen, die die Konsequenzen von Entscheidungsalternativen beeinflussen, ist immer fragmentarisch; ${ }^{68}$

68 Die moderne Organisationstheorie hat überdies darauf aufmerksam gemacht, dass das menschliche Wissen und die menschliche Rationalität nicht nur begrenzt sind, sondern dass daher viele Handlungen nicht auf der unmittelbaren Wahrnehmung von Daten und Kausalgesetzen der realen Welt, sondern auf kulturell geformten und sozial konstruierten Überzeugungen basieren (vgl. Scharpf 2000, S. 51). Die „soziale Konstruktion der Realität" (Berger/Luckman) ist daher von entscheidender Bedeutung. Was Akteure für wahr und real halten, bestimmt ihr Handeln. 
- einen Überblick über mögliche Handlungsalternativen und deren Konsequenzen zu erhalten;

- Handlungsalternativen zueinander und zu Zielsetzungen zuzuordnen und zu bewerten;

- zu beeinflussen oder zu kontrollieren - oder auch nur vorherzusagen -, was andere potenzielle Akteure unter unterschiedlichen Bedingungen unternehmen werden.

Reale Menschen oder Organisationen sind nach Simon also keine Optimierer oder Maximierer, sondern satisficer, sie suchen nur so lange nach Alternativen, bis sie eine Lösung gefunden haben, die „gut genug“, good enough erscheint. March/Simon (1976) verdeutlichen dies an dem bekannten Stecknadelbeispiel: Auf der Suche nach einer Nadel im Heuhaufen wird die Suche nach dem Auffinden der ersten Nadel abgebrochen, die ausreichend spitz ist (befriedigende Lösung), um damit zu nähen. Es wird nicht versucht, die spitzeste Nadel zu finden (optimale Lösung), da das Individuum weder weiß, ob es noch eine spitzere Nadel gibt, noch wie lange die Suche dauern würde. Was jeweils die befriedigende Lösung ist, hängt vom jeweiligen Anspruchsniveau ab, welches mit der Erfahrung der Individuen variiert. Kann ein gegebenes Anspruchsniveau über längere Zeit nicht erreicht werden, senkt der Entscheider seine Ansprüche. Dieser Zusammenhang gilt auch in umgekehrter Richtung. Man kann sein Anspruchsniveau auch erhöhen, wenn gegebene Niveaus ohne Probleme erreicht werden. Eine weitere Entscheidungshilfe neben dem satisficing ist die selektive Perzeption der Situation. Individuen und Organisationen gehen in der Regel von einer vereinfachten Definition der Situation aus, sie nehmen nur einen Ausschnitt der Wirklichkeit wahr (vgl. ausführlich auch Bogumil/Schmid 2002, S. 40f.).

\subsubsection{Inkrementalismus und Durchwursteln}

Genau dies ist auch der Ausgangspunkt der Lehre vom Inkrementalismus oder vom "Sich-Durchwursteln" (the science of muddling through), wie sie sich selbstironisch bezeichnet. Sie möchte in erster Linie realistisch beschreiben und erklären, wie politisch-administrative Systeme handeln und entscheiden, dabei wird zwischen den beiden Elementen Regierung und Verwaltung nicht weiter differenziert. Untersuchungsgegenstand ist die „Administration“. Politik ist nach dieser Auffassung ein kontinuierlicher Prozess der Problemlösung. Diese Aufgabe muss von der jeweiligen Administration erfüllt werden. Zentrale Fragestellung ist daher: Wie werden politische Entscheidungen getroffen und wie sollten sie sinnvollerweise getroffen werden?

Ansatzpunkt der Argumentation ist auch hier die Auseinandersetzung mit einem rationalen oder synoptischen Modell der Entscheidungsfindung, wie es z.B. von Anhängern umfassender, langfristiger Planung vorgeschlagen wird. Charles E. Lindblom, der bekannteste Vertreter und „Erfinder“ der Lehre vom 
„Sich-Durchwursteln“ (vgl. Lindblom 1959, deutsch 1975 und Braybrooke/Lindblom 1972, zusammenfassend Migone/Howlett 2015, Wegrich 2015b), behauptet, dass Administrationen nicht, wie im rationalen Modell unterstellt, zunächst Ziele und Zwecke des politischen Handelns genau ermitteln und festlegen, dann sämtliche Strategien (Mittel) zur Erreichung dieser Ziele erarbeiten und schließlich die für das gesetzte Ziel beste oder geeignetste Strategie auswählen (Zweck-Mittel-Abwägung). Eine umfassende, „rationale" Planung in diesem Sinne ist nicht nur unmöglich; es ist auch verfehlt, dieses Modell nur anzustreben. Stattdessen ist für administratives Handeln eine Strategie der unkoordinierten kleinen Schritte (disjointed incrementalism) bei der Entscheidungsfindung rational und sinnvoll (vgl. zu dieser Darstellung Böhret/Jann/Junkers/Kronenwett 1979, S. 263).

Charles E. Lindblom (geb. 1917) promovierte 1945 an der an der University of Chicago in Volkswirtschaft, anschließend wechselte er 1946 an die Yale University, wo er bei seiner Emeritierung 1980 eine Professur für Wirtschafts- und Politikwissenschaft innehatte. Wie die Arbeiten von Robert Dahl, Anthony Downs, Thomas Schelling u.a. steht auch Lindbloms Werk an der Schnittstelle zwischen den Wirtschafts- und Sozialwissenschaften. Zum einen behandelt er Fragen der Politikwissenschaft mit dem konzeptionellen Fokus und den Methoden der Wirtschaftswissenschaften, zum anderen bringt er politikwissenschaftliche Standpunkte in die Wirtschaftswissenschaften ein.

Von bahnbrechender Bedeutung ist vor allem Lindbloms 1959 veröffentlichter Aufsatz über die „Wissenschaft des Sich-Durchwurstelns“ (The Science of Muddling Through), der ihn zu einem der in den Sozialwissenschaften am häufigsten zitierten Autoren machte. Zur Blütezeit politisch-administrativer Planung und des Glaubens an durch immer bessere technische Systeme unterstützte, rationale Entscheidungssysteme zeichnet Lindblom ein völlig neues Bild politischer und administrativer Entscheidungsprozesse. Sie seien weniger als planvolle, rationale Problemlösungsaktivitäten zu verstehen, sondern vielmehr als das Ergebnis eines sich Durchwurstelns in kleinen, unkoordinierten, inkrementellen Schritten, die bestenfalls in einer schrittweisen Problemlösung mit kleinen Verbesserungen münden. Dieses Modell des „muddling through “versteht Lindblom zum einen deskriptiv als eine Beschreibung tatsächlicher Entscheidungsprozesse, zum anderen aber auch normativ, als die einer Demokratie angemessene Entscheidungsform, da sie die schrittweise Einbindung verschiedener gesellschaftlicher Interessen erlaube.

Der Begriff der inkrementalen Politik, der sich auch im Deutschen durchgesetzt hat, bedeutet dabei nach Lindblom:

- Politische oder administrative Entscheidungen orientieren sich normalerweise am Status quo und streben nur jeweils kleine Verbesserungen an (marginale Veränderungen). 
- Dadurch wird eine schrittweise Problemlösung (sequenzielle Problemverarbeitung) erreicht. Probleme sollen und können nicht endgültig "gelöst“ werden, sondern es wird nach einem angemessenen Fortschritt in einer vermutlich erfolgversprechenden Richtung gesucht.

- Dabei werden nicht adäquate Mittel für feststehende Zwecke gesucht, sondern die Zwecke werden umgekehrt an vorhandene Mittel angepasst. Die wichtigsten Impulse für politische Entscheidungen ergeben sich nicht aus übergeordneten Zielen, sondern aus aktuellen Missständen und vorhandenen Mitteln.

Inkrementale Politik ist weiterhin dadurch charakterisiert, dass sie „disjointed“, d.h. unkoordiniert abläuft. Dies bedeutet:

- Problemlösung wird nicht von irgendwelchen Zentren hierarchisch kontrolliert, sondern findet unkoordiniert, durch eine Vielzahl von Entscheidungseinheiten statt, wie dies für hochgradig arbeitsteilige Organisationen typisch ist;

- dadurch werden Interessen und Informationen von verschiedenen Seiten berücksichtigt;

- die Beiträge dieser verschiedensten Entscheidungsträger werden durch einen Prozess der gegenseitigen Verhandlung und Anpassung (partisan mutual adjustment) zusammengebracht und ausgeglichen, bei dem keine Entscheidungseinheit andere dominieren oder unterdrücken kann (siehe oben negative Koordination).

Der hier beschriebene Vorgang der politischen Entscheidungsfindung als ein Prozess der permanenten und partiellen Anpassung an veränderte Problemlagen und relevante Interessen ist nach Ansicht Lindbloms das in der politisch-administrativen Realität vorherrschende Verhalten. So wie hier beschrieben, handeln Administrationen wirklich.

Das klassische Beispiel für inkrementale Entscheidungen ist der jährliche Budgetprozess, wie ihn der amerikanische Politikwissenschaftler Aaron Wildavsky in einer berühmten Studie über den Budgetprozess in den USA beschrieb (1984, zuerst 1964). Auch beim Budget wird nicht jedes Jahr wieder bei politischen Zielen und Handlungsalternativen begonnen, sondern das alte Budget ist Ausgangspunkt der politischen Verhandlungen und wird dann, je nach "partisan mutual adjustment" marginal fortgeschrieben. Ein aktuelles Beispiel ist die Corona-Krise, in der es kontinuierliche Verhandlungen und Anpassungen zwischen verschiedenen Politikbereichen, Bund, Ländern und Gemeinden gab, und immer wieder ein ,schrittweises' Vorgehen mit der Möglichkeit der Korrektur angemahnt wurde.

Nach Ansicht Lindbloms ist diese Strategie der unkoordinierten kleinen Schritte des „Sich-Durchwurstelns" nicht nur eine realistische Beschreibung politisch-ad- 
ministrativer Prozesse, sondern das für ein demokratisches System angemessene und sinnvolle Verhalten. Lindblom sieht u. a. folgende Vorteile:

- Die menschliche Entscheidungsfähigkeit und Möglichkeit, Informationen aufzunehmen und zu verarbeiten, wird nicht überfordert: „etwas ,vernachlässigen', heißt etwas ,überhaupt erst analysierbar machen'; nach Vollständigkeit zu streben, bedeutet zuweilen, sich ein unbrauchbares Ergebnis einzuhandeln“ (Braybrooke/Lindblom 1972, S. 150).

- Weil das Wissen über die Zukunft prinzipiell unsicher ist, ist die schrittweise Veränderung des Status quo der sicherste Weg, um Risiken zu vermeiden. Da Veränderungen jeweils nur geringfügig sind, können sie nach dem Prinzip des „Versuch und Irrtum“ (trial and error) vorgenommen werden. Wenn eine Entscheidung sich als falsch erweist, sie z. B. andere als die gewünschten Folgen hat, kann sie leicht wieder revidiert werden (vgl. hierzu auch Karl Poppers Begriff des piecemeal social engineering; ders. 1945, S. 139ff.).

- Die durch inkrementales Vorgehen getroffenen Entscheidungen zeichnen sich dadurch aus, dass sie weitgehend akzeptiert werden, da sie ja nur geringfügige Änderungen vornehmen und durch den Prozess der gegenseitigen Anpassung die verschiedensten Interessen berücksichtigen. Daraus folgt aber auch, dass die Politik richtig ist, denn „Einigung auf eine bestimmte Politik ist (...) der einzige brauchbare Test für die Richtigkeit einer Politik“(Lindblom 1975, S. 168).

- Schließlich entspricht die inkrementale Politik in idealer Weise einer pluralistischen Gesellschaft. Durch die dezentralisierte Entscheidungsfindung wird eine Vielzahl der vorhandenen gesellschaftlichen Interessen berücksichtigt und somit eine gewisse Vollzähligkeit der in der Gesellschaft vorhandenen Werte erreicht. Inkrementalismus ist daher die dem Pluralismus angemessene Form der Entscheidungsfindung. Ähnlich wie im ökonomischen Marktmodell entsteht durch die marginalen und unkoordinierten Entscheidungen der isolierten Entscheidungsträger ein gesellschaftlich optimaler Zustand.

Inkrementale Politik bedeutet dabei nicht, dass auf jegliche Analyse oder Planung verzichtet wird. Es geht nur darum, den Stellenwert rationaler Analyse in politisch-administrativen Entscheidungsprozessen realistisch einzuschätzen und einzuordnen. In Anlehnung an Lindblom unterscheidet Wildavsky daher zwei „reine“ Modelle, nämlich „synoptische“ und ,inkrementale“ Politik (Wildavsky 1979, S. 114 ff.).

Die klassische Institution synoptischer Entscheidung ist der Plan, der idealtypisch umfassende Information erfordert, die zentral und hierarchisch erhoben und aggregiert wird. Grundlage „richtiger“ Pläne ist die „richtige“ Erkenntnis und umfassende Analyse. Faktische und normative Irrtümer müssen möglichst ver- 
mieden werden, da sie in umfassenden Plänen erhebliche negative Auswirkungen haben. Das Kriterium, ob ein umfassender Plan angenommen werden sollte, ist daher, ob er „richtig“ ist, auf richtiger Erkenntnis basiert.

\begin{tabular}{lcc}
\hline & „reines synoptisches Modell“ & „reines inkrementales Modell“ \\
\hline Institution & Plan & Markt \\
& & Politik \\
\hline Information & umfassend & begrenzt \\
\hline Akteure & zentral & dezentral \\
& hierarchisch & unabhängig \\
\hline Grundlage & Erkenntnis & Verhandlung \\
& Analyse & Anpassung \\
\hline Irrtum & Vermeidung & Korrektur \\
\hline Kriterium & Richtigkeit & Übereinstimmung \\
\hline
\end{tabular}

Abb. 50 Modelle synoptischer und inkrementaler Politik

Quelle: eigene Darstellung nach Wildavsky 1979, S. 123

Demgegenüber geht es im reinen inkrementalen Modell prinzipiell nur um begrenzte Information, die dezentral und unabhängig voneinander in vielen Einheiten erhoben und verarbeitet wird. Grundlage inkrementaler Politik sind Verhandlung und Anpassung, Irrtümer können nicht vermieden werden, es geht im Gegenteil darum, sie möglichst schnell zu machen, um sie schnell korrigieren zu können. Das Kriterium inkrementaler Politik ist die Übereinstimmung der beteiligten Akteure, und die typischen Institutionen sind sowohl der Markt wie die Politik.

Wildavsky behauptet nun keinesfalls, dass politisch-administrative Entscheidungsprozesse nur und ausschließlich inkremental ablaufen oder ablaufen sollten. Aber er insistiert, dass diese Prozesse immer aus Interaktionen (Verhandlungen, Abstimmungen) und Analysen (Erkenntnis) bestehen und dass politische Interaktionen und Verhandeln nicht durch rationale Analyse und Erkenntnis ersetzt werden können und sollen. Als Ergebnis hält er eine Daumenregeln bereit: Das Verhältnis von 1/3 Analyse (Policy Analyse) und 2/3 Interaktion scheint ihm eine sinnvolle und anzustrebende Mischung bei Entscheidungen über staatliche Policies zu sein.

Offenkundig gibt es in Politik und Verwaltung nicht nur inkrementelle Entscheidungen, sondern gelegentlich auch umfassende, zum Teil sehr schnelle Veränderungen von Policies. In der wissenschaftlichen Diskussion werden diese eher seltenen, aber wichtigen Umwälzungen mit dem Konzept des punctuated equlibrium beschrieben und erklärt. Der Ansatz hat allerdings ausdrücklich nicht das Ziel, inkrementelle 
Erklärungen zu verdrängen oder zu widerlegen, sondern es geht darum, besser zu verstehen, wo und warum es gelegentlich doch zu Richtungswechseln kommt. Es geht, in den Worten von Baumgartner darum "to understand the nature of policy change more generally“ (als Übersicht Baumgartner et.al. 2014, Hegelich/Knollmann 2014).

\subsubsection{Garbage Can}

In der gleichen Tradition sog. „nicht-rationaler" Entscheidungstheorien, die allerdings keineswegs behaupten, dass in Politik und Verwaltung irrational entschieden würde, sondern die die Aufmerksamkeit von normativen aber unrealistischen Modellen auf realistische Prozesse und die ihnen innewohnenden Rationalität lenken wollen, steht ein von James March und Johan P. Olsen entwickeltes Modell, das sie durchaus selbstironisch Garbage Can oder auf Deutsch Mülleimer-Modell von Entscheidungen genannt haben (zuerst Cohen/March/Olsen 1972, March/Olsen 1976, zusammenfassend Jann 2015).

Ausgangspunkt sind Entscheidungs- und Lernprozesse unter den Bedingungen von Mehrdeutigkeit und Unklarheit (ambiguity). Solche Situationen sind durch beschränktes Wissen und Kontroversen sowohl über die zugrunde liegenden Probleme, die zur Lösung geeigneten Technologien und gleichzeitig durch inkonsistente oder sogar unoperationale Zielsysteme gekennzeichnet. Anhand einer klassischen, natürlich stark vereinfachenden Matrix von Thompson/Tuden kann dies verdeutlicht werden (Thompson/Tuden 1959, de Leon 1998):

\begin{tabular}{|c|c|c|c|}
\hline & & \multicolumn{2}{|c|}{ Ziele } \\
\hline & & $\begin{array}{l}\text { klar } \\
\text { eindeutig }\end{array}$ & $\begin{array}{l}\text { widersprüchlich } \\
\text { konfliktär }\end{array}$ \\
\hline \multirow{2}{*}{$\begin{array}{l}\text { Instrumente/ } \\
\text { Technologien }\end{array}$} & sicher & $\begin{array}{l}\text { Berechnung } \\
\text { Bürokratie } \\
\text { Hierarchie }\end{array}$ & $\begin{array}{l}\text { Verhandlung } \\
\text { Repräsentation } \\
\text { Abstimmung }\end{array}$ \\
\hline & unsicher & $\begin{array}{l}\text { Beurteilung } \\
\text { Kollegium } \\
\text { Profession }\end{array}$ & $\begin{array}{l}\text { Abstimmung } \\
\text { Netzwerk } \\
\text { Anarchie }\end{array}$ \\
\hline
\end{tabular}

Abb. 51 Handeln in mehrdeutigen Situationen

Quelle: eigene Darstellung nach Thompson/Tuden 1959

In Situationen, in denen sowohl über die zu erreichenden Ziele wie auch über die dafür adäquaten Instrumente und Technologien Einigkeit besteht, also wenn es 
darum geht, eine Straße zu bauen oder Wohngeld auszuzahlen, sind technokratische, bürokratische und hierarchische Entscheidungsregeln angemessen.

Es gibt aber auch Situationen, in denen zwar klar ist, wie ein Problem zu lösen wäre, aber durchaus nicht, welche Ziele vorrangig verfolgt werden sollen. Ein Beispiel sind typische Verteilungsprobleme, wenn also klar ist, dass und wie eine Straße gebaut werden soll, aber umstritten ist wo? Oder wenn etwa die Gesundheitskosten gesenkt werden müssen, aber durchaus kontrovers ist, wer dafür aufkommen soll (Ärzte, Patienten, Pharmakonzerne, Krankenhäuser). Diese Probleme werden i. d. R. durch Verhandlungen gelöst, in denen die wichtigsten Akteure und Interessen repräsentiert sind. Ein klassisches Beispiel sind Entscheidungen in politischen Gremien wie Parlamenten oder Kommunalvertretungen. Wenn es keine Einigung gibt, wird am Ende abgestimmt.

Eine ganz andere Situation entsteht, wenn zwar die zu erreichenden Ziele eindeutig und unkontrovers sind, allerdings unklar und kontrovers bleibt, wie diese Ziele zu erreichen wären. Das klassische Beispiel ist hier wiederum die Gesundheit, aber auch Bildung gehört dazu: Wenn wir zum Arzt gehen, wollen wir gesund werden. Wie dies zu erreichen ist, ist aber unklar. Genau aus diesem Grund haben in der Corona-Krise die Virologen eine so entscheidende Rolle gespielt. Probleme dieser Art werden i.d. R. „professionell“ gelöst, d.h. sie werden einer Profession zugewiesen, der man das notwendige Wissen zutraut, die man aber gleichzeitig von den Folgen ihrer Handlungen entlastet und die ggf. kollektiv entscheidet. Ärzte können nur bei schweren Kunstfehlern verklagt werden; Studenten, die bei ihren Hochschullehrern nichts lernen, haben kaum eine Chance sich zu wehren. Bei Entscheidungsbedarf gibt es ein Kollegium von Ärzten oder eine Prüfungskommission von Professoren.

Besonders interessant sind schließlich Situationen, in denen es weder Einigkeit über die Ziele gibt noch darüber, was eigentlich zu tun sei und wirksam sein könnte. Genau dies sind die Probleme, die typischerweise der Politik und damit natürlich auch der Verwaltung zugewiesen werden. Über das allgemeine Ziel der Bildungs- und Forschungsförderung besteht noch Einigkeit, aber welche konkreten Ziele dabei vorrangig sind (Förderung der Allgemeinbildung oder der Eliten, Natur- oder Sozialwissenschaften) und wie dies erreicht werden soll (Privatschulen und Privatuniversitäten, Studiengebühren oder mehr staatliches Geld), bleibt kontrovers und unklar.

Genau hier setzt die Garbage Can Theorie an. Sie behauptet, dass viele nur einigermaßen komplexe Entscheidungsprozesse und Problemlösungen aus vier weitgehend voneinander unabhängigen „Strömungen“" (Cohen/March/Olsen 1990, S. 333) bestehen: 
- Lösungen, die nach Problemen suchen, auf die sie angewendet werden könnten (man denke nur an neue Kommunikationstechnologien, Reorganisationsvorschläge, Führungsinformationssysteme u. ä.),

- Teilnehmer, die nach Gelegenheiten suchen, in relevanten Entscheidungsprozessen eine gewichtige Rolle zu spielen,

- Situationen, die es erlauben oder erfordern, Entscheidungen zu treffen oder einen Entscheidungsprozess abzuschließen (z. B. regelmäßige Gelegenheiten wie das jährliche Budget, aber auch unverhoffte Krisen), und schließlich auch

- Probleme, die ganz unabhängig von vorhandenen Lösungen, Aktivisten und Gelegenheiten darauf warten, bearbeitet zu werden.

Diese vier grundlegenden Entscheidungsströme, so diese Theorie, existieren weitgehend unabhängig voneinander, ihre Interaktionen sind stark situationsabhängig und deshalb nur schwer vorhersehbar. Konkrete Entscheidungsprozesse ähneln daher besagtem Mülleimer, in dem die vier Ströme mehr oder weniger zufällig zusammentreffen. Offensichtlich gibt es verschiedene Mülleimer, und welche Lösungen mit welchen Akteuren wann zusammenkommen, hängt u. a. davon ab, welches Etikett die einzelnen Mülleimer tragen (daher die Bedeutung von Organisationsstrukturen).

Kingdon (1995) hat diesen Ansatz auf Prozesse der Politikformulierung und des Agenda Setting übertragen und dabei besonders auf die Gelegenheitsstrukturen, die „windows of opportunity “ für innovative und kontroverse Entscheidungen verwiesen. Zudem betont er die Rolle von "policy entrepreneurs", also Akteuren, die in der Lage sind, solche unklaren Entscheidungssituationen für Ihre Interessen auszunutzen. In der wissenschaftlichen Diskussion hat dieser Ansatz inzwischen unter der Überschrift „multiple streams“ Karriere gemacht (vgl. zusammenfassend Rüb 2014).

Auch wenn die Garbage Can Theorie gelegentlich wie eine Karikatur konkreter Entscheidungsprozesse erscheinen mag (damit der Inkrementalismustheorie sehr ähnlich), so lenkt sie doch unsere Aufmerksamkeit auf unbestreitbare Phänomene, wie z.B. darauf, dass in Organisationen keineswegs immer aufgrund rationaler Analysen entschieden wird, sondern dass vorhandene Lösungen, wenn sich die Gelegenheit ergibt, gern auf neue Probleme angewendet werden. So hat die Elbe-Flut im Jahre 2002 dazu geführt, dass zum einen seit langem kontroverse Ausbaupläne von den Gegnern gestoppt, zum anderen vorhandene Pläne zum Ausbau und zur Reorganisation des Katastrophenschutzes endlich durchgesetzt werden konnten (Radunz 2003). Die Vorschläge der Hartz-Kommission waren keineswegs allesamt neu, aber der „Vermittlungsskandal“ in der Bundesanstalt für Arbeit schuf ein „window of opportunity“, eine einmalige Entscheidungsgelegenheit zur Verbin- 
dung seit langem diskutierter Lösungen mit neuen Problemen und vor allem mit wichtigen Akteuren (Weimar 2003, Schmid 2003). Und vielleicht wird die aktuelle Coronakrise die Gelegenheit bieten, schon länger existierende Vorschläge einer stärkeren Digitalisierung von Verwaltungsprozessen künftig stärker durchzusetzen.

Insgesamt lenken alle diese Theorien unsere Aufmerksamkeit darauf, dass arbeitsteilige, bürokratische Organisationen sehr konfliktär sind, insbesondere gilt dies für öffentliche Organisationen. Sie leben mit permanenter Unsicherheit und immanenten Zielkonflikten. Daher widmen sie sich zu unterschiedlichen Zeiten unterschiedlichen Problemen, sie beschäftigen sich gleichzeitig in verschiedenen Teilen der Organisation mit widersprüchlichen Zielen. Während die Verkehrsabteilung versucht, Autobahnen zu bauen, versucht die Umweltabteilung, sie zu verhindern. Öffentliche Organisationen versuchen diese Unsicherheiten aufzufangen und organisatorisch zu verarbeiten, z.B. durch Arbeitsteilung, Regelbindung, Hierarchie und negative Koordination. Eine weitere neue Organisationstheorie spricht in diesem Zusammenhang von der "organization of hypocrisy“, von der organisierten Heuchelei, und weist darauf hin, dass "talk, decisions and actions“ in Organisationen allenfalls "lose gekoppelt" sind (Brunsson 1989). Das worüber Organisationen in der Öffentlichkeit reden, entspricht nicht notwendigerweise dem, was sie programmatisch entscheiden, und dies wiederum wird oft auch gar nicht umgesetzt (siehe nächster Abschnitt).

Um die eigene Informations- und Konfliktlösungskapazität nicht permanent zu überlasten, handeln solche Organisationen, wann immer es geht, auf der Grundlage von Routine, von Standard Operating Procedures, anstelle von gründlicher Analyse, Planung und Prognose. Genau dies ist der tiefere Grund der bekannten bürokratischen Maximen „das haben wir schon immer so gemacht" und „das haben wir noch nie so gemacht".

Ansätze wie Garbage Can oder Hypocricy verdeutlichen die engen Beziehungen, die sich in den letzten Jahren zwischen Verwaltungswissenschaft und modernen Organisationstheorien entwickelt haben. Ausgehend von Simons Theorie der begrenzten Rationalität (bounded rationality) waren dabei vor allem skandinavische Verwaltungswissenschaftler führend (als bester Überblick Christensen et.al. 2020, s. a. Jann 2006a, Laegreid 2020), gleichzeitig gibt es enge Verbindungen zu neo-institutionalistischen Theorien und generell zur „institutionalistischen Wende“ in der Politikwissenschaft (grundlegend March/Olsen 1983, dies. 2006, auf Deutsch Kaiser 2001). 


\subsubsection{Politikdurchführung und Evaluation}

\subsubsection{Implementation}

Die Entscheidung für ein politisches Programm, z. B. die Verabschiedung eines Gesetzes oder auch des jährlichen Budgets, garantiert noch kein praktisches Handeln der durchführenden Instanzen, d.h. in aller Regel der öffentlichen Verwaltung, und erst recht nicht den Erfolg eines Programms. Die Phase der Durchführung oder Umsetzung eines beschlossenen Programms, der Vollzug von Gesetzen und Rechtsverordnungen, die Ausführung von politisch beschlossenen Maßnahmen, wird in der Politikwissenschaft als Implementation bezeichnet. Die besondere Bedeutung dieser Phase des politischen Prozesses besteht darin, dass politisches und administratives Handeln durch Gesetze, Handlungsprogramme, Zielvorgaben usw. nicht endgültig steuerbar ist und daher in dieser Phase politische Programme und deren Intentionen verzögert, verändert oder sogar vereitelt werden können (siehe zum Folgenden Jann/Wegrich 2014 m. w. A., zur Weiterentwicklung der Implementationsforschung Adam/Knill 2018). Elemente dieser Phase sind Entscheidungen über

- Programmkonkretisierung (Wie und durch wen soll das Programm ausgeführt werden? Wie ist das Gesetz zu interpretieren?),

- Ressourcenbereitstellung (Wie werden Finanzen verteilt? Welches Personal führt das Programm durch? Welche Organisationseinheiten sind mit der Durchführung betreut?), und natürlich ganz besonders

- Einzelfälle (welche Genehmigung wird erteilt, wer bekommt welche Unterstützung, welche Straße wird gebaut? etc.).

Die „Entdeckung“ und Problematisierung der Implementationsphase kann als eine der wichtigsten Innovationen der Politik- und Verwaltungsforschung in den siebziger Jahren gelten. Die Implementation politischer Programme, die Durchführung von Gesetzen war zuvor als im Prinzip unproblematisch angesehen worden. Gesetze werden bekanntlich vom Parlament „verabschiedet“, und damit war das Problem für den Gesetzgeber erledigt. Hintergrund dieser Implementationsignoranz war wiederum eine problematische Interpretation der weberschen Bürokratietheorie, nach der die Bürokratie gesetzte Regeln effektiv, präzise, verlässlich etc. umsetzt. Weber war durchaus klar, dass dieses idealtypische Merkmal der Bürokratie in der Wirklichkeit durchaus prekär ist, aber zur Legitimation administrativen Handelns ist es von großem Wert. Wenn die Verwaltung „nur Gesetze umsetzt“, so die normative und empirische Interpretation der Bürokratietheorie, ist sie von den Folgen und Konflikten ihres Handelns weitgehend entlastet. 
Mit der bahnbrechenden Studie von Pressman/Wildavsky (1973; zusammenfassend Wegrich 2015b) zur Implementation sozialpolitischer Programme in den USA (mit dem berühmten Untertitel „How Great Expectations in Washington are Dashed in Oakland; Or Why it's Amazing that Federal Programs Work at all...") wurde gezeigt, dass die Durchführungsphase nicht nur Teil des politischen Prozesses ist, sondern häufig die entscheidende Phase, in der sich der Erfolg oder Misserfolg eines politischen Programms herausstellt, und in der auch kontroverse politische Entscheidungen erst getroffen oder zumindest konkretisiert werden. Wenn in Einzelfallentscheidungen festgelegt wird, wer welche Genehmigungen, staatlichen Förderungen oder Infrastrukturleistungen bekommt oder nicht bekommt, geht es um grundlegende politische Entscheidungen: Wer bekommt was, wann, wo und warum? (So die klassische Definition von Politik durch Lasswell).

Nach dem deutschen Rechtsstaatsverständnis ist Verwaltungshandeln rechtlich programmiert, d.h. alle Maßnahmen und Entscheidungen der Verwaltung müssen rechtmäßig sein und können daher auch vor Verwaltungsgerichten überprüft werden. Die Verwaltung wird also durch Gesetze, Rechtsverordnungen und durch autonome Satzungen (etwa der Kommunen) gesteuert (legislative Steuerung). Es gilt der Vorrang des Gesetzes (Gesetze sind anderen Rechtsquellen außer der Verfassung übergeordnet) und der Vorbehalt des Gesetzes (nach der Wesentlichkeitstheorie des Bundesverfassungsgerichts bedürfen wesentliche staatliche Maßnahmen einer gesetzlichen Grundlage). Die typische Einzelfallentscheidung ist daher der öffentlich-rechtliche Verwaltungsakt, allerdings gibt es auch andere Handlungsformen, etwa öffentlich-rechtliche Verträge oder privatrechtliches Handeln der Verwaltung (etwa städtebauliche Verträge, die im Bereich der Stadtsanierung eingesetzt werden oder Arbeits- und Werkverträge, vgl. zusammenfassend Becker 1989, S. 449ff.).

Bezüglich der rechtlichen Programmierung des Verwaltungshandelns werden zwei grundsätzliche Formen unterschieden (grundlegend Luhmann 1966)

- Konditionalprogramme, die ein bestimmtes Verwaltungshandeln festlegen, wenn bestimmte Bedingungen erfüllt sind. In der einfachsten Form sind dies „Immer-Wenn-Dann-Programme“, die der Verwaltung, zumindest theoretisch, überhaupt keinen Entscheidungsspielraum überlassen. Beispiele wären etwa das Passgesetz oder das Gesetz über Personalausweise: Immer, wenn die notwendigen Unterlagen beigebracht werden, muss ein Pass ausgestellt werden. Es gibt im Prinzip keinen Handlungsspielraum der Verwaltung. ${ }^{69}$

69 Handlungsspielräume gibt es indes auch hier bei der Beurteilung der für die Verwaltungsleistung notwendigen Unterlagen, wie dies z. B. in der Flüchtlingskrise beim Agieren 
- Final- oder Zweckprogramme lassen demgegenüber den jeweiligen Entscheidern in der Verwaltung wesentlich größere Freiräume. Hier sind nur die angestrebten Zwecke und Ziele festgelegt, während die Mittel zur Erreichung dieser Zwecke erst noch von der Verwaltung ausgewählt werden müssen (allenfalls sind zu beachtende Restriktionen, etwa finanzielle Mittel vorgegeben). Beispiele wären etwa staatliche oder kommunale Fachplanungen oder z. B. Wirtschaftsförderungsprogramme, ein klassisches Beispiel ist der $\$ 1$ des Bundesraumordnungsgesetzes: „Das Bundesgebiet ist in seiner allgemeinen räumlichen Struktur einer Entwicklung zuzuführen, die der freien Entfaltung der Persönlichkeit in der Gemeinschaft am besten dient. Dabei sind die natürlichen Gegebenheiten sowie die wirtschaftlichen, sozialen und kulturellen Erfordernisse zu beachten“ (\$1 BRauOG).

Es ist offenkundig, dass diese beiden reinen Formen in der Realität kaum anzutreffen sind, sondern dass reales Verwaltungshandeln durch eine Kombination konditionaler und finaler Programmierung gesteuert wird (für den Versuch der Bildung weiterer Typen s. Becker 1989, S. 451). Eine wichtige Rolle spielen in diesem Zusammenhang z.B. unbestimmte Rechtsbegriffe (etwa „unbillige Härte“), die von der Verwaltung interpretiert werden müssen, oder der Verweis auf „pflichtgemäßes Ermessen“ der Verwaltung. Gerade auch in Deutschland, wo aufgrund der rechtsstaatlichen und bürokratischen Tradition die rechtliche und formale Programmierung besonders ausgeprägt ist (siehe oben 4.1 Überregelung und Verrechtlichung), verfügt also die Verwaltung, selbst die Ordnungs- und Eingriffsverwaltung, über erhebliche Handlungsspielräume. Frido Wagener hat z. B. wiederholt darauf hingewiesen, dass aufgrund der Vielzahl und Komplexität der rechtlichen Regelungen die öffentliche Verwaltung ohnehin nur einen Teil davon überhaupt beachten kann, sie also "pragmatische Vorschriftenreduktion im Vollzug" betreibt, sich diejenigen Regelungen aussucht, die gerade passen und sich dabei im „permanenten Verfassungsbruch“" befindet (Wagener 1979).

Die für die Verwaltungswissenschaft entscheidende Frage ist, wie die Verwaltung diese Handlungsspielräume nutzt. Ähnlich wie in den USA begann auch in Deutschland mit den ersten Enttäuschungen der Reformpolitiken der sozial-liberalen Koalition Anfang der siebziger Jahre der Aufschwung der Implementationsforschung. Zunächst nahm man dabei eine Perspektive ein, die später als „Gesetzgeberperspektive“ oder „Top-down“-Ansatz bezeichnet wurde. Die Implementationsprozesse wurden vor allem unter dem Aspekt des Grades der zielgenauen Umsetzung der

von Ausländerämtern deutlich geworden ist (vgl. Bogumil/Burgi u.a. 2018 und weiter unten). 
auf übergeordneter (meist zentralstaatlicher) Ebene definierten Politikziele analysiert und die Gründe für Abweichungen von diesen Zielen in verwaltungsinternen Prozessen sowie der Interaktion der Vollzugsbehörden mit den betroffenen Adressaten im Rahmen von Verhandlungs- und Konfliktbeziehungen analysiert. Die theoretische Perspektive basierte dabei auf einem klassischen hierarchischen Verständnis politischer Steuerung (vgl. grundlegend Mayntz 1980a, dies. 1983, Wollmann 1980, zusammenfassend Mayntz 1987).

Gefragt wurde also nach Vollzugsdefiziten (warum werden bestimmte Regelungen nicht angewendet? z. B. in der Umweltpolitik, Mayntz 1977), nach Zielverschiebungen (warum werden in der Umsetzung andere Ziele als ursprünglich intendiert verfolgt? etwa in der Wirtschaftsförderung, Böhret/Jann/Kronenwett 1982) oder nach sozialen Selektivitäten (welche Gruppen werden benachteiligt, welche warum bevorzugt? vgl. auch Mayntz 1997, S. 217). In die Aufmerksamkeit der Forscher gerieten Probleme des Kontakts zwischen Verwaltung und Publikum, die bereits in den siebziger Jahren zu einem umfassenden Forschungsprogramm zur „bürgernahen Verwaltung“ führten (Kaufmann 1979, Hegner 1978, Grunow 1982) und die Probleme der Zusammenarbeit und Steuerung zwischen Behörden und in Policy-Networks zum Gegenstand hatten.

Vollzugsdefizite, z.B. die ineffektive (Ziele werden verfehlt) oder ineffiziente Durchführung von Gesetzen und politischen Programmen (Aufwand und Ertrag stehen in einem problematischen Verhältnis zueinander) wurden so in Merkmalen der Programme (etwa widersprüchliche oder unklare Ziele, problematische Ziel-Mittel-Annahmen, Überregelung, Verrechtlichung) oder der Implementationsstrukturen (unklare, komplizierte Organisation, ungeeignetes Personal, komplexe und langwierige Verfahren, unzureichende Finanzen) verortet, zunehmend aber auch in Merkmalen des Regelungsumfeldes, die von der Verwaltung gar nicht zu beeinflussen sind.

Zugleich leiteten diese empirischen Studien somit einen Perspektivenwechsel ein (insgesamt zur Entwicklung der Implementationsforschung Winter 2012). Der Implementationsprozess wurde immer weniger als hierarchische (top down) Steuerung durch übergeordnete Einheiten betrachtet, sondern zunehmend als gemeinsamer Lern- und Aushandlungsprozess. Die Verwaltung erhält ihre Entscheidungsprämissen nicht nur „von oben“, durch Gesetze und hierarchische Weisungen, sondern auch „von unten“ und „von der Seite“, also durch Kunden, Klienten, Bürgerinitiativen, Interessengruppen.

Einerseits erkannte man die zentrale Bedeutung der Vollzugsbehörden auf den unteren Ebenen („Street Level Bureaucracy“, grundlegend Lipsky 2010, Hupe/Hill/ Bbuffat 2015) und richtete den Blick auf die Interaktionsbeziehungen mit den eigentlichen Adressaten politischer Programme (vgl. z. B. Wollmann 1983). Andererseits 
sah man zunehmend die Verbindung zwischen den internen und externen Akteuren eines Politikfeldes auf den verschiedenen Ebenen und begann Policy-Making als alle Phasen umfassenden Verhandlungsprozess innerhalb netzwerkartiger Beziehungen zu verstehen - womit letztendlich von der Annahme abgerückt wurde, dass ein staatliches Steuerungszentrum hierarchisch in gesellschaftliche Handlungsfelder intervenieren kann. Stattdessen richtete man den Blick auf das Zusammenspiel verschiedener Akteure in einem Policy-Subsystem (Sabatier 1987, 1993), das zwar durch unterschiedliche Interessen und asymmetrische Einflussverteilung zwischen den Beteiligten geprägt ist, jedoch insgesamt einen systemischen Zusammenhang kollektiver „Politikproduktion“ konstituiert (Jansen/Schubert 1995).

Eine weitere Erkenntnis war, dass die Implementationsphase ganz entscheidend durch die zur Anwendung kommenden Instrumente des politischen Programms geprägt wird (König/Dose 1992, Braun/Giraud 2003). Neben regulativen Instrumenten (Ge- und Verbote, Genehmigungspflichten) werden u. a. finanzielle (positive und negative Anreize, Leistungsprogramme), die direkte Bereitstellung von staatlichen Leistungen (harte und weiche Infrastruktur, also z. B. sowohl Schulen und Universitäten wie Lehrer und Professoren) und Informationsinstrumente (Aufklärung, Propaganda) unterschieden. Die Untersuchungen zeigten, dass die verschiedenen Instrumente spezifische Implementationsprobleme aufweisen. Während regulative Politik vor allem mit dem Kontrollproblem und möglichen Widerständen auf Seiten der Adressaten verbunden ist (vgl. z. B. Bohne/Hucke 1980), sind Anreizprogramme, wie Scharpf bereits 1983 am Beispiel der Arbeitsförderung zeigte, der Gefahr von „Mitnahmeeffekten“ (Unternehmen nehmen Fördergelder für ohnehin geplante Investitionen oder Arbeitsplätze in Anspruch), d. h. der ineffizienten Mittelverteilung ohne Steuerungseffekte, ausgesetzt.

In jüngster Zeit sind einige experimentellen Studien zum Problembereich der „soziale Selektivität“ entstanden (Grohs/Adam/Knill 2016, Adam/Grohs/Knill 2020), die thematisieren, dass in Abhängigkeit vom Antragsteller (Geschlecht, Migrationshintergrund) die Schnelligkeit und Qualität der Verwaltungsleistung variieren, obwohl eigentlich Gleichbehandlung beabsichtigt sein sollte. Auch Beobachtungen hinsichtlich des Verwaltungsbeitrags bei der Nicht-Inanspruchnahme von Leistungen z. B. im Bereich des Bildungs- und Teilhabepaketes ${ }^{70}$ oder bei der Einbürgerung (vgl. hierzu Bogumil/Burgi u. a. 2018) gehören zu diesem

70 Die im Jahr 2011 eingeführten „Leistungen für Bildung und Teilhabe“ sind eine der Sozialleistungen mit der geringsten Inanspruchnahme in Deutschland (neben Wohngeld und Grundsicherung im Alter). Gleichzeitig streut im interkommunalen Vergleich die Inanspruchnahme der BuT-Leistungen mit Spannweiten der Inanspruchnahme von 1,9\% bis $91,3 \%$ enorm (Grohs 2019). 
Themenkomplex der sozialen Selektivität von Verwaltungshandels. Weiterhin werden Untersuchungen zu Mitnahmeeffekten beim Konjunkturpaket (Schneider/ Grohs/Knill 2011) angesichts der Corona-Krise wieder ein aktuelles Thema werden.

\subsubsection{Verhandelnde Verwaltung und kooperativer Staat}

Die Ergebnisse der empirischen Verwaltungs- und Implementationsforschung haben zu einem von der klassischen hierarchischen Bürokratie abweichenden Bild der modernen Verwaltung geführt, das unter dem Schlagwort der verhandelnden oder kooperativen Verwaltung zusammengefasst wird und schließlich sogar zu einem veränderten Bild des Staates als "kooperativer Staat" geführt hat (grundlegend Benz 1994, Dose 1997, Schuppert 2000, S. 110ff.). Andere Autoren sprechen in diesem Zusammenhang auch vom ,informalen Verwaltungshandeln“ das schliesslich zu einem „informalen Rechtsstaat“ führe (Bohne 1981).

Grundlegendes Merkmal der kooperativen Verwaltung ist der weitgehende Verzicht auf die Anwendung von Zwang. Kooperativ handelt die Verwaltung also z.B., wenn sie mit Adressaten Entscheidungen aushandelt und mit ihnen entweder informelle Absprachen trifft oder Verträge schließt. So war ein Ergebnis der Implementationsforschung, dass Behörden etwa im Bereich des Umweltschutzes oder der Gewerbeaufsicht auf regulative Instrumente (Verbote, Gebote bis hin zu Geldstrafen oder Betriebsschließungen) verzichten, obwohl diese Maßnahmen ausdrücklich im Gesetz vorgesehen sind. Stattdessen versuchen die zuständigen Behörden sich z. B. mit „Umweltsündern“ zu einigen, bis wann bestimmte Missstände abgestellt werden. Der Hintergrund ist die Abwägung unterschiedlicher Ziele. Die Schliessung einer Betriebsstätte mag umweltpolitisch sinnvoll sein, hat aber negative arbeitsmarktpolitische, sozialpolitische und andere ungewollte Folgen. Um einen Ausgleich dieser unterschiedlichen Interessen zu erreichen, ist kooperatives Verwaltungshandeln sinnvoller und angemessener als der eigentlich juristisch vorgesehene Vollzug z. B. mit Hilfe von Zwangsmitteln. Ein weiteres Verständnis der kooperativen Verwaltung umfasst daher auch Fälle, in denen zuständige Behörden Verhaltensweisen vorübergehend oder dauerhaft dulden, obwohl sie diese aufgrund von Gesetzen verhindern könnten (vgl. zum Folgenden Benz 2003c).

Normalerweise beruht eine solche Kooperation zwischen Verwaltung und den Adressaten von politischen Programmen auf Verhandlungen, in denen Verwaltungsbehörden mit Entscheidungsbetroffenen eine Einigung suchen. Nicht selten enden diese mit einem formalen Verwaltungsakt, der die Ergebnisse der Verhandlungen festlegt. Dieser ist dann zwar der Form nach ein Hoheitsakt der Verwaltungsbehörde, tatsächlich stellt er aber eine Vereinbarung zwischen gleichberechtigten Partnern dar. Oft beruht eine Einigung, wie oben erwähnt, auf Tauschgeschäften, die durch Verbindung verschiedener Entscheidungsmaterien möglich werden, wobei die Ver- 
waltung in einem Bereich und die Adressaten ggfs. in einem ganz anderen Bereich Konzessionen machen. Auf die Problematik solcher Vereinbarungen, wenn nicht zusammengehörende Sachverhalte verkoppelt werden, macht Benz aufmerksam: Kooperation kann z.B. die Rechte und Belange „Dritter“ verletzen, die nicht an Verhandlungen beteiligt sind (siehe auch Dose 2009).

Eine weitere Bedeutung kooperativen Verwaltungshandelns ergibt sich bei der Erstellung personenbezogener Dienstleistungen, die ohne Mitwirkung der Adressaten nicht erfolgreich sein können. Wenn es z. B. darum geht, Arbeitslose in Arbeit zu vermitteln oder sie fortzubilden, oder die Ansiedlung bzw. den Ausbau von Unternehmen zu unterstützen, ist dies nur in enger Kooperation mit den jeweiligen Adressaten und Klienten der Verwaltung möglich.

Der mit dem Konzept der kooperativen Verwaltung eng verbundene Begriff des „Kooperativen Staates" entstammt der politischen Theorie und der Staatsrechtslehre, die damit von überholten Vorstellungen einer Überordnung des Staates über die Gesellschaft bzw. einer Trennung von Staat und Gesellschaft abrücken (Ritter 1979, grundlegend Scharpf 1992). Das Bild des kooperativen Staates beschreibt also die Beobachtung, dass der moderne Staat in vielen Aufgabenbereichen auf Verhandlungen und Zusammenarbeit mit gesellschaftlichen Gruppen oder Organisationen angewiesen ist, und zwar sowohl bei der Formulierung wie auch gerade bei der Umsetzung politischer Programme und Maßnahmen.

\subsubsection{Evaluation}

Schließlich sind Behörden und Verwaltungen bei Entscheidungen über die Wirkungen und Auswirkungen politischer Programme beteiligt, und damit bei der Frage nach deren Veränderung, Verbesserung oder ggfs. sogar Beendigung (Termination). In der verwaltungswissenschaftlichen Forschung hat sich dafür der Begriff der Evaluation eingebürgert. Evaluation ist dabei von den traditionellen „Kontrollschleifen“" (Wollmann 2003) im deutschen Regierungs- und Verwaltungssystem zu unterscheiden (siehe oben unter 3.8), nämlich

- der Überprüfung von Ordnungsmäßigkeit und Wirtschaftlichkeit durch Rechnungshöfe,

- der Überprüfung der Rechtsmäßigkeit des Verwaltungshandelns durch Verwaltungsgerichte,

- der verwaltungsinternen Kontrolle durch hierarchische Überordnung und (Rechts- und Fach-) Aufsicht sowie

- der politischen Kontrolle durch Parlament und Öffentlichkeit (siehe aber unten). 
Staatliche Programme und Aktivitäten und damit auch Verwaltungshandeln, sollen einen Beitrag zur Lösung oder zumindest Verarbeitung gesellschaftlicher, sozialer und ökonomischer Problemlagen leisten. Diese angestrebten Wirkungen politischer Programme und administrativer Aktivitäten stehen in der Evaluation im Vordergrund, nicht die Kontrolle einzelner Akteure. Mit Evaluation wird dabei einerseits die Phase des politischen Prozesses bezeichnet, in der die Ergebnisse der Implementation bewertet (evaluiert) werden. Zugleich hat sich andererseits die Evaluationsforschung als ein Teilbereich der Verwaltungs- und Policy-Forschung entwickelt, die ihren Ausgangspunkt in der Frage nach den - intendierten und nicht-intendierten - Wirkungen öffentlicher Aktivitäten hat und dabei inzwischen alle Phasen des politischen Prozesses thematisiert (ausführlich Wollmann 2003, Sager/Hinterleitner 2014; zum Folgenden auch Jann/Wegrich 2014).

Die wissenschaftliche Diskussion über Evaluation und Wirkungsforschung hatte sich in den USA wiederum in Verbindung mit den politischen Kontroversen über Sinn und Erfolge der sozialpolitischen Programme der "Great Society“ der sechziger Jahre herausgebildet. Auch sie wurde sehr schnell in Deutschland rezipiert und führte zu einer umfangreichen Diskussion z. B. über Möglichkeiten und Grenzen der Wirkungsforschung und „experimenteller Politik“ (Derlien 1976, Wollmann/ Hellstern 1978). Inzwischen ist Evaluationsforschung einer der erfolgreichsten und umfangreichsten Zweige angewandter Sozialforschung (Bussmann/Klöti/Knöpfel 1997, Vedung 1999).

Unter Evaluation wird gemeinhin die wissenschaftliche oder zumindest systematische Untersuchung der - intendierten oder nicht-intendierten - Wirkungen und Auswirkungen politischer und administrativer Interventionen verstanden. Dabei interessiert nicht nur die jeweilige Zielerreichung (Erfolgskontrolle), sondern auch positive oder negative Effekte und Nebenwirkungen, z. B. auch in anderen als den intendierten Bereichen (Wirkungsforschung). In der wissenschaftlichen Diskussion werden eine Reihe unterschiedlicher Ansätze und Methoden der Evaluation und der Evaluationsforschung unterschieden (Wollmann 2003, S.338ff.):

- Bei der ex-post (oder summativen) Evaluation wird im Nachhinein, nach Abschluss eines Programms oder einer Maßnahme, untersucht, welche Wirkungen und Nebenwirkungen eingetreten sind (Wirkungsanalyse) bzw. ob und in welchem Umfang die intendierten Ziele erreicht wurden (Erfolgskontrolle).

- Demgegenüber hat die ex-ante Evaluation die Aufgabe, Wirkungen, Nebenwirkungen und Ursache-Wirkungszusammenhänge eines künftigen Handlungsprogramms vorab abzuschätzen (pre-assement). Im deutschen wird hier oft der Begriff Gesetzesfolgenabschätzung verwendet (vgl. Böhret/Konzendorf 2002, 
Veit 2008), in diesen Zusammenhang gehören auch die klassischen Methoden der Kosten-Nutzen-Analyse.

- Formative (oder on-going) Evaluation findet begleitend zur Durchführung der jeweiligen Programme und Maßnahmen statt, um möglichst frühzeitig im Rahmen der „Rückkopplung“ von Ergebnissen Korrekturen zu ermöglichen. Im deutschen hat sich hierfür der Begriff der Begleitforschung etabliert, die mehr oder weniger analytisch distanziert bis hin zur Form einer sich aktiv einmischenden Aktionsforschung stattfindet.

- Weiter werden Effektivitäts- und Effizienz- (oder Wirtschaftlichkeits-) Untersuchungen unterschieden. Bei ersteren geht es in der Form eines Soll-Ist-Vergleichs um den Zielerreichungsgrad bezüglich der Outputs (Leistungen der Verwaltung), bis hin zu den direkten Wirkungen (Impacts) und weitergehenden gesellschaftlichen Auswirkungen (Outcomes), bei letzterer um das Verhältnis zwischen Inputs (finanzielle, personelle, organisatorische Ressourcen) und Ergebnissen (Outputs und Impacts).

- Unter Monitoring versteht man in diesem Zusammenhang eine deskriptiv-analytische, auf kausale Interpretationen und Erklärungen weitgehend verzichtende Beobachtung relevanter Ergebnisse und Resultate, möglichst mit Hilfe standardisierter Indikatoren, also eine routinemässige, permanente und systematische Sammlung von vergleichbaren Daten (Sager/Hinterleitner 2014, S. 439).

- Controlling ist schließlich vor allem in Verbindung mit dem Neuen Steuerungsmodell populär geworden, insbesondere zur Überprüfung der dort postulierten Output-orientierten Steuerung der Verwaltung durch Produkte, Kennzahlen, Berichtswesen und generell performance measurement (vgl. zu den verschiedenen Konzepten die Beiträge in Veit u.a. 2019; auch Kuhlmann/Bogumil/ Wollmann 2004). Auch beim Controlling geht es „strategisch“ um Ziel- und Effektivitätssteigerung und "operativ" vor allem um Effizienz. Der zentrale Unterschied zur Evaluation besteht darin, dass es sich um interne Instrumente der jeweiligen Organisationen, Behörden etc. handelt und die Ergebnisse des Controllings direkt zur Steuerung des Planungs- und Leistungsprozesses und zur Leistungssteigerung erhoben und eingesetzt werden sollen.

Evaluationen können Monitoring-Daten oder auch Resultate des Controllings einbeziehen. Klassische wissenschaftliche Evaluationsuntersuchungen versuchen allerdings möglichst nach den strikten Regeln empirischer Sozialforschung (Experimente, Quasi-Experimente, counter-factuals, vergleichende Fallstudien etc.) zu klären, ob beobachtbare Veränderungen - intendierte wie nicht-intendierte Wirkungen - auf die politischen Programme, Projekte und Maßnahmen, oder aber auf andere Faktoren kausal zurückzuführen sind (als Lehrbuch Vedung 1999). 
Dabei ergeben sich eine ganze Reihe schwieriger Methodenprobleme, denn zum einen sind die Zielsetzungen politischer Programme oft alles andere als klar und eindeutig (siehe oben 4.3.1.4 „Garbage Can“), zum anderen ist es äußerst schwierig, bestimmte Ergebnisse auf bestimmte politisch-administrative Faktoren „kausal“ zurückzuführen (siehe ebd. „unklare Instrumente und Technologien“).

Wenn z.B. die Wirkung arbeitsmarkt- oder regionalpolitischer Massnahmen evaluiert werden soll, ist selbst der deutliche Rückgang der jeweiligen regionalen Arbeitslosigkeit ein sehr problematischer Indikator. Die geringere Arbeitslosigkeit könnte nämlich etwa durch Abwanderung oder durch konjunkturelle Einflüsse beeinflusst sein. Selbst wenn Erfolge nachweisbar sind, bedeutet dies nicht, dass sie auch tatsächlich auf staatliche Maßnahmen und Programme zurückzuführen sind.

Dennoch ist deutlich, dass Evaluierung in Zukunft eine zunehmende Bedeutung haben wird. Ein Beispiel ist die durch die internationale PISA-Studie der Lernerfolge von Grundschülern ausgelöste Diskussion. Hier wurde deutlich, dass zum einen unterschiedliche Schulsysteme sehr unterschiedliche materielle Resultate hervorbringen (z. B. in Bezug auf Lese- und Rechenfähigkeit), dass diese unterschiedlichen Ergebnisse aber nicht einfach auf den Ressourceneinsatz zurückgeführt werden können (Deutschland schneidet eher schlecht ab, obwohl deutsche Lehrer besonders gut bezahlt werden, deutsche Klassen nicht besonders groß sind und insgesamt das Bildungssystem überdurchschnittlich kostspielig ist). Evaluationsuntersuchungen sind also besonders geeignet, um beliebte und einfache Erklärungsmuster zu hinterfragen („Das einzige was unseren Schulen fehlt sind mehr Geld und mehr Lehrer ...").

Ein besonderes Problem wirft die Evaluation verwaltungspolitischer Maßnahmen auf, also etwa der Nachweis der Erfolge oder Misserfolge der Verwaltungsmodernisierung der letzten Jahrzehnte. Das Bedürfnis für verlässliche Evaluationen in diesem Bereich ist groß, denn natürlich ist es wichtig zu wissen, welche positiven und negativen Veränderungen die Verwaltungsmodernisierung tatsächlich hervorgerufen hat, jenseits der vollmundigen Versprechungen der professionellen Berater. Gerade den Protagonisten des New Public Management wird immer wieder vorgeworfen, dass ausgerechnet eine Reformbewegung, die darauf besteht, der öffentliche Sektor müsse von einer Input- zu einer Output-Steuerung kommen, seine Kosten und vor allem seinen Nutzen transparent machen und diese Informationen in kontinuierlichen Feedback-Prozessen nutzen - und gelegentlich zu unterstellen scheint, dies sei alles ganz einfach, wenn man nur richtig wolle -, bisher nicht in der Lage sei, systematisch über die Erfolge der stattgefundenen Reformbemühungen zu berichten. Die Philosophie des NPM ist bisher eher selten auf die eigenen Aktivitäten angewendet worden (siehe aber Pollitt/Bouckaert 2017, als erste Bestandsaufnahmen für 
Deutschland Jann u. a. 2004 und besonders Bogumil/Groh u. a. 2007; als Überblick Seyfried 2019b, siehe auch unten 5.2.3).

Die Evaluation staatlicher Programme und Aktivitäten findet allerdings - unabhängig von der Bedeutung wissenschaftlicher oder systematischer Evaluation - als Teil des Prozesses politischer und administrativer Auseinandersetzungen „schon immer" statt, wie sowohl das PISA- wie das Verwaltungsreform-Beispiel verdeutlichen. Von der wissenschaftlichen kann so die administrative Evaluation durch die Verwaltung und die politische Evaluation durch Akteure innerhalb der politischen Arena, zu denen auch die Öffentlichkeit gerechnet werden muss, unterschieden werden (zur Frage der Evaluationsstandards vgl. Grohs/Piesker 2019). Nicht nur wissenschaftliche Studien, sondern z. B. Regierungsberichte und die öffentliche Debatte, nicht zuletzt Verlautbarungen der jeweiligen Opposition, sind typische Elemente und Ergebnisse dieser Art von Auseinandersetzungen, bei der es natürlich auch immer um Zielerreichung, intendierte und nicht-intendierte Wirkungen geht.

Evaluationen sind daher im besonderen Maße mit der politischen Rationalität administrativer Handlungen verbunden. Dies betrifft nicht nur die interessengefärbte Interpretation der Ergebnisse und Wirkungen. Die Möglichkeit systematischer Evaluation wird auch durch unklare Zieldefinitionen eingeschränkt, die ihrerseits in der Anreizstruktur von Regierungen und politisch Verantwortlichen begründet sind - denn genaue Zieldefinition birgt das erhebliche Risiko des deutlichen späteren Scheiterns (s. die Probleme der ersten rot-grünen Regierung mit ihrem ursprünglichen quantifizierten Ziel zur Reduzierung der Arbeitslosigkeit).

In einem immer noch äußerst lesenswerten Beitrag macht Aaron Wildavsky (1979, S. 212ff.) darauf aufmerksam, dass dies ein allgemeines Phänomen sei, dass also die „self-evaluating organization“, die sich ständig selbst evaluierende und damit prinzipiell infrage stellende Organisation ein Widerspruch in sich sei. Es sei naiv anzunehmen, dass Organisationen ein unmittelbares Interesse an Evaluationen haben, weder „von innen“, also durch spezialisierte und herausgehobene Evaluationseinheiten, noch von ,außen“, also durch andere Organisationen mit vermutlich anderen Interessen und Prioritäten. Evaluationen sind „weapons in the political wars", Waffen in politischen und fachlichen Auseinandersetzungen, und zwar sowohl zwischen Organisationen auf der makro-politischen Ebene wie mikro-politisch innerhalb von Organisationen. Evaluationen sind möglich und notwendig, aber sie sind alles andere als selbstverständlich und einfach nur technisch schwierig. Sie werfen nicht nur erhebliche methodische, sondern mindestens ebenso komplizierte politische Probleme auf, d.h. sie müssen in kontinuierlichen politischen Auseinandersetzungen durchgesetzt, durchgeführt und interpretiert werden. In den Worten von Wildavsky: 
"I started out thinking it was bad for organizations not to evaluate, and I ended up wondering why they ever do it" (Wildavsky 1979, S. 212).

Ergebnis eines politischen Evaluationsprozesses kann schließlich auch die „Terminierung" (Beendigung) eines politischen Programms sein. In Deutschland wird auch der Begriff „Aufgabenkritik“ für die Terminierung von Policies bzw. den Abbau oder die zeitliche Befristung staatlicher Aktivitäten verwendet (Dieckmann 1977). Dabei erscheint die zunächst nahe liegende Möglichkeit, dass zu Grunde liegende gesellschaftliche Probleme als gelöst betrachtet und eine Fortsetzung daher als unnötig eingeschätzt werden, nur als eine unwahrscheinliche, zudem schwierig durchzusetzende, Variante der Terminierung. Eher können finanzielle Engpässe (beispielsweise in der Arbeitsförderung) oder Gelegenheitsfenster (z. B. im Zuge eines Regierungswechsels oder durch Krisen, etwa Fukushima im Bereich der Kernkraft) Auslöser für die Terminierung bestimmter Policies und Aktivitäten sein (vgl. zu den Beispielen Kernenergie und Kohlesubventionen in Deutschland Heyen 2011).

\subsection{Politik und Verwaltung}

\subsubsection{Dichotomie oder politisch-administratives System}

Die Unterscheidung bzw. der Zusammenhang zwischen Politik und Verwaltung ist ein klassisches, wenn nicht das klassische Thema der Verwaltungswissenschaft, die sich nun einmal mit öffentlicher Verwaltung beschäftigt, und natürlich auch ein kontroverses Thema der Verwaltungspraxis (siehe Kapitel 2.4.1 und 2.4.2 und zum Folgenden Jann/Veit 2020). In der verwaltungswissenschaftlichen Literatur können idealtypisch zwei Sichtweisen unterschieden werden.

Auf der einen Seite gibt es die Vorstellung einer grundsätzlichen Dichotomie zwischen Politik und Verwaltung, die auf die bahnbrechenden Beiträge von Woodrow Wilson (1887) und Max Weber (1921) zurückgeht. Beide beschreiben Politik und Verwaltung als unterschiedliche soziale Systeme, die jeweils ihre eigene Rationalität aufweisen. In der Politik geht es um Macht, Legitimität, Leidenschaft, die Durchsetzung von Interessen und Politikinhalten, während es in der Verwaltung um Sachkunde, Professionalität, Legalität und die technische Durchführung von Politik gehen soll. Verwaltung ist in diesem Sinne nicht eigenständig, sondern ,nur Instrument der Politik. Allerdings hatten bereits Wilson und Weber erhebliche Zweifel, dass professionelle Bürokratien von Amateurpolitikern kontrolliert werden können. Weber verwies auf die Überlegenheit der Bürokraten aufgrund zweier Ar- 
ten von Wissen: Fachwissen (die überlegene Expertise spezialisierter Bürokraten) und Dienstwissen (ihr prozedurales und institutionelles Wissen über die Funktionsweise der öffentlichen Verwaltung). Verwaltungen können also eigenständige Machtzentren sein, deren Herrschaft schwer zu kontrollieren ist.

Diese Sichtweise ist auch die Grundlage moderner principal-agent Theorien, in denen die Prinzipale, also Politiker, aufgrund unvermeidlicher Informationsasymmetrien erhebliche Probleme haben, das Verhalten ihrer Agenten, also der Verwaltung zu kontrollieren. Das Verhältnis zwischen Politikern und Bürokraten ist daher durch systematisches Misstrauen, Opportunismus, durch Machtspiele und ewige blame-games gekennzeichnet, in denen die eine Seite jeweils die andere für Misserfolge und Probleme verantwortlich macht. Im Extremfall geht der ökonomisch geprägte Prinzipal-Agent Ansatz davon aus, dass Bürokraten grundsätzlich nicht zu trauen ist, da diese sich entweder vor Arbeit drücken oder eigene Ziele verfolgen, etwa Budgetmaximierung (siehe zu diesen „Theorien rationalen Handelns in der Politikwissenschaft“ Braun 2013; umfassend zu principal-agent Gilardi/Braun 2002, kritisch Pierre/Peters 2017).

Auf der anderen Seite gibt es eine eher empirisch geprägte Auffassung, die eine strikte Trennung von Politik und Verwaltung für unmöglich hält. Die Orthodoxie der Dichotomie von Politik und Verwaltung brach in den USA spätestens mit den Erfahrungen des New Deal und des Zweiten Weltkrieges zusammen. Die damals entwickelte Grundthese, dass sowohl Politik wie Verwaltung bei der Konzipierung und Formulierung von Politikinhalten, wie bei deren Durchsetzung und Evaluation zusammenarbeiten und beteiligt sind, wurde von der empirischen Verwaltungsforschung seit dieser Zeit immer wieder, immer differenzierter und immer überzeugender belegt.

Zum einen hat die die empirische Verwaltungsforschung gezeigt, wie groß der „Entscheidungsbeitrag der Bürokratie“ (Scharpf 1973b, S. 16) im Rahmen der Programmformulierung ist, d.h. welche Probleme verdrängt, welche Ziele vernachlässigt und welche Handlungsalternativen in der Phase der Entscheidungsvorbereitung bereits aussortiert werden, ehe formale politische Strukturen wie Parlamente mit dem Entscheidungsprozess befasst sind. Sie zeigt auch, wie stark die Verwaltung in gesellschaftliche Interessenstrukturen eingebunden ist. Administrative Interessenvermittlung (Lehmbruch 1987) ist eines der herausragenden Merkmale moderner demokratischer Staaten. Zum anderen zeigt die empirische Verwaltungsforschung, insbesondere die Implementationsforschung (vgl. 4.3.2), dass der Vollzug von Programmen zu einem nicht unerheblichen Teil „politischer Prozess“ ist, dass viele Fragen durch die formelle politische Entscheidung noch nicht entschieden wurden und die eigentliche politische Auseinandersetzung erst während des Vollzuges beginnt (Mayntz 1980a, 1983). 
Die politikwissenschaftliche Verwaltungsforschung hatte auf diese Erkenntnis zunächst mit einer radikalen Negierung der Trennung von Politik und Verwaltung reagiert (erst in den USA, ab den siebziger Jahren auch in Deutschland). In der Folge sprach man fortan vom „politisch-administrativen System“, abgekürzt PAS, oder sogar vom Kürzel RV für „Regierung und Verwaltung“. Aber indem man die politischen Funktionen der Verwaltung betont und Politik und Verwaltung konsequent gemeinsam betrachtet, wird die Frage, wie denn das Verhältnis zwischen Politik und Verwaltung konkret ausgestaltet ist, einfach verdrängt. Das politisch-administrative System mag eine Metapher sein, mit der man die Wirklichkeit in Ministerien einfangen kann (siehe nächster Abschnitt), aber sie versagt vor der Größe und gleichzeitigen Vielgestaltigkeit der öffentlichen Verwaltung. Das theoretische Konzept des nahtlosen und undifferenzierten politisch-administrativen Systems, in dem es keinen Unterschied zwischen Politik und Verwaltung gibt, trägt wenig zum Verständnis der empirisch offenkundigen unterschiedlichen Funktionen, Rollenzuweisungen, Rekrutierungen und Einstellungen in öffentlichen Organisationen bei.

Weder eine klare Dichotomie noch ein einheitliches politisch-administratives System, können offensichtlich die Komplexität der Wirklichkeit einfangen. Dazu braucht es präzisere Unterscheidungen. Die einfachste Unterscheidung setzt an der jeweiligen Rekrutierung und Legitimationsbasis an. Unterschieden wird zwischen gewählten Politikern (etwa Parlamentarier, Minister, oder auch kommunale Wahlbeamte), die jederzeit abgewählt werden oder zumindest Wahlen und damit ihre Stellung verlieren können, und ernannten (oder angestellten) Bürokraten (also allen Mitarbeitern der Verwaltung), die entweder als Beamte eine Lebensstellung haben, und ansonsten durch das normale Arbeitsrecht geschützt sind. Die problematische Unterscheidung der diesen Gruppen jeweils zugeordneten Funktionen kann dann am besten anhand von vier gängigen Interpretationen des Verhältnisses und der Kooperation zwischen beiden illustriert werden: ${ }^{71}$

- Nach Interpretation I, der ältesten und einfachsten Theorie über das Verhältnis von Politik und Verwaltung machen Politiker Politik und Bürokraten verwalten. Die einen treffen Entscheidungen und die anderen führen sie nur aus. Dieses Bild der Arbeitsteilung, auch wenn es wahrscheinlich nie vollständig zugetroffen hat, ist dennoch ein wichtiges Element der Mythologie über die neutrale,

71 Diese Interpretationen, im Original „Images“ genannt, gehen zurück auf die international vergleichende Untersuchung der Einstellungen von Parlamentariern und Bürokraten von Aberbach/Putnam/Rockman 1981. 
unpolitische Rolle der Bürokraten im politischen System und damit im Prozess der Formulierung und Umsetzung von Politik.

- Interpretation II geht davon aus, dass sowohl Politiker wie Beamte an politischen Entscheidungen beteiligt sind (etwa: wie soll das Gesetz aussehen? wer bekommt wann, was, wo und warum?), dass sie aber unterschiedliche Beiträge liefern. Beamte liefern Fakten und Wissen, Politiker bringen Interessen und Werte ein. Die einen sind für die neutrale Expertise zuständig, während die anderen für die politische Sensibilität verantwortlich sind.

- Interpretation III hingegen behauptet, dass sowohl Bürokraten wie Politiker „Politik machen“, also z. B. verhandeln und am Interessenausgleich teilnehmen, dass sie aber unterschiedliche Aufgaben wahrnehmen. Während Politiker breite, diffuse aber auch spezifische Interessen artikulieren und so dafür sorgen, dass sich im politischen Prozess etwas bewegt, also als Beweger und Energielieferanten auftreten, sind Beamte damit beschäftigt, zwischen wohldefinierten und etablierten Interessen zu vermitteln, d.h. sie sorgen eher für Kontinuität und Ausgleich. Nach diesem Bild sind Politiker eher parteiisch, engagiert, idealistisch und sogar ideologisch, während Bürokraten eher als vorsichtig, praktisch, pragmatisch und distanziert gelten.

- Interpretation IV geht schließlich davon aus, dass sich Bürokraten und Politiker immer mehr angleichen, beide artikulieren und verhandeln Interessen und sind mit der gesellschaftlichen Umwelt eng vernetzt. Angenommen wird, dass eine Entwicklung hin zu reinen „Mischlingen“ zwischen Politikern und Bürokraten feststellbar sei.

Diese Interpretationen oder Bilder, die anhand des Verhältnisses zwischen „Bürokraten“ und „Politikern“ in Ministerien entwickelt wurden (Aberbach/Putnam/ Rockman 1981), treffen vermutlich nicht nur für diesen Bereich zu. Auch in Kommunalverwaltungen sind Beamte und Angestellte damit beschäftigt, Interessen auszugleichen oder auch Interessen, die sonst vielleicht nicht artikuliert werden, überhaupt erst in den Prozess des Verwaltungshandelns einzubringen. Die verschiedenen Interpretationen können vereinfacht in der folgenden Abbildung zusammengefasst werden. Offenkundig leistet Verwaltung in aller Regel mehr, als nur politische Entscheidungen hierarchisch durchzuführen (wie es der Webersche Idealtypus nahelegt, auch wenn Max Weber nie dieser Illusion aufgesessen ist). Letztendlich ist auch die Durchführung keine Domäne der Verwaltung mehr, sondern unterliegt (zunehmenden) politischen Einflüssen, und auch im Konzept der kommunalen Selbstverwaltung war diese Trennung schon immer aufgehoben (siehe unten). 


\begin{tabular}{lllcc}
\hline & \multicolumn{4}{c}{ Interpretation } \\
\hline & I & II & III & IV \\
\hline Politikdurchführung & V & V & V & V \\
\hline Politikformulierung & P & G & G & G \\
\hline Interessenausgleich & P & P & G & G \\
\hline Interessenartikulation & P & P & P & G \\
\hline
\end{tabular}

$\mathrm{V}=$ Verwaltung, $\mathrm{P}=$ Politik, $\mathrm{G}=$ Gemeinsam

Abb. 52 Interpretation des Verhältnisses von Politik und Verwaltung

Quelle: eigene Darstellung nach Aberbach/Putnam/Rockman 1981, S. 239

\subsubsection{Bürokraten und Politiker: Funktionale und parteipolitische Politisierung}

Gerade auch im internationalen Vergleich werden die unterschiedlichen Auffassungen der Rollen von Politikern und Bürokraten deutlich. In praktischer und normativer Hinsicht ähnelt der erste Idealtyp - das ,instrumentalistische“ Konzept mit seiner Betonung eines neutralen und a-politischen öffentlichen Dienstes - dem sogenannten „Westminster-Modell“, das aus dem (ehemaligen) Commonwealth oder den skandinavischen Ländern bekannt ist. Dort sind Ministerialbeamte prinzipiell „unpolitisch“ und werden auch nach Regierungswechseln i.d. R. nicht ausgewechselt. Gleichzeitig spielen Prinzipal-Agent-Probleme eine wichtige Rolle in politischen und theoretischen Diskussionen, genauso wie Konflikte zwischen politischen Beratern und permanenten Bürokraten (Hustedt/Houlberg Salomonsen 2014). Einfache und, wie viele argumentieren, naive Modelle des New Public Management haben diese instrumentelle Sichtweise übernommen, indem sie argumentierten, Politik sollte sich nur mit dem „Was“, also den Zielen der Politik befassen, während das „Wie“ der Verwaltung überlassen werden sollte (vgl. 5.2.4).

Die zweite Sichtweise, die davon ausgeht, dass es zumindest in Führungspositionen keine unpolitischen Bürokraten gibt und solche Stellen daher politisch besetzt werden sollten, entspricht am ehesten dem US-System mit seiner großen Anzahl von politisch besetzten Positionen in der Verwaltung (Lewis 2012). Aber z. B. auch in vielen süd- und osteuropäischen Ländern zollen Regierungen oft dem Ideal von neutralen und a-politischen Beamten Lippenbekenntnisse, während sie eine große Anzahl von ihnen nach Wahlen oder aus anderen politischen Gründen ersetzen (Meyer-Sahling 2008).

Die politische Praxis in Deutschland liegt zwischen diesen beiden Idealtypen, obwohl nach der überkommenen traditionellen Auffassung in den alten hegelianisch 
inspirierten Staatstheorien, die zumindest bis in die erste Hälfte des 20. Jahrhunderts prominent und dominant waren, die Bürokratie unpolitisch ist und über der Politik steht, da sie den Staat "als sittliche Idee“ verkörpert. Nur der öffentliche Dienst, also die Bürokratie, kann, so die Annahme, das Gemeinwohl garantieren und durchsetzen, notfalls auch gegenüber Politikern, Interessengruppen und Parteien, die lediglich Sonderinteressen vertreten (Böhret/Jann/Kronenwett 1982, S. 257ff.; Jann 2003). Diese Ideologie des unpolitischen, neutralen, nur am Gemeinwohl interessierten Beamten wurde allerdings spätestens in der Nazi-Zeit brutal diskreditiert. Bereits in der Weimarer Republik waren viele Spitzenbeamte entschieden antidemokratisch, unterstützten rechte Parteien und Ideen und spielten später eine wichtige Rolle beim Aufstieg und den Verbrechen der Nationalsozialistischen Deutschen Arbeiterpartei (NSDAP). Viele traten der NSDAP bei, nachdem Deutschland 1933 ein Einparteienstaat geworden war $^{72}$. Der berühmte liberale Verfassungsrechtler, Gustav Radbruch, hatte die Vorstellung des a-politischen, neutralen Beamten bereits 1930 als „Lebenslüge des Obrigkeitsstaates“ charakterisiert.

Aber auch vor diesen verheerenden Erfahrungen war eine einfache Unterscheidung zwischen Politik und Verwaltung noch nie ein bestimmendes Merkmal des deutschen politischen und administrativen Systems. In Deutschland waren schon früh institutionelle Merkmale entwickelt worden, um Verbindungen zwischen den beiden Sphären herzustellen, insbesondere die Konzepte des "politischen Beamten“ auf der ministeriellen und des „Wahlbeamten“ auf der lokalen Ebene (siehe unten).

In Deutschland ist die Verwaltung daher nicht prinzipiell a-politisch oder neutral, sondern durchaus politisiert. Dabei ist es hilfreich, analytisch zwei Dimensionen von Politisierung zu unterscheiden. Zum einen „funktionale Politisierung“, also die Fähigkeit, alle möglichen Aufgaben der Politikgestaltung wahrzunehmen, also von Politikformulierung, Zieldefinition, Verhandlungen innerhalb und außerhalb der Regierung bis hin zur Interaktion mit Bürgern und Interessengruppen (siehe Abb. 52 oben). Dabei geht es auch, aber nicht nur darum, die Erreichung der politischen Zielsetzungen der Hausleitung voranzutreiben. Dies bedeutet wiederum, die Position der Hausspitze nicht nur zu kennen und zu vertreten, sondern bei neu auftretenden Fragen eine solche Position auch treffsicher zu antizipieren oder sogar zu beeinflussen (und damit konstruktiv zu gestalten), noch bevor sie überhaupt gebildet werden konnte. Die zweite Dimension ist die „Parteipolitisierung“, also welche Rolle parteipolitische Orientierung z.B. für Stellenbesetzungen und Karrieren in der Verwaltung spielt, bis hin zu einer Ämterpatronage (vgl. zum Folgenden Jann/Veit 2020).

72 Bereits 1934 waren zwei Drittel aller deutschen Spitzenbeamten in den Ministerien Mitglieder der NSDAP, dieser Anteil stieg bis auf mehr als $90 \%$ in 1939 (Jann/Veit 2020). 


\subsubsection{Ministerialverwaltung des Bundes}

In der deutschen Verwaltungswissenschaft wird, ausgehend von den skizzierten Idealtypen der Beziehung zwischen Politik und Verwaltung, schon des längeren mit den Idealtypen des klassischen und des politischen Bürokraten gearbeitet (vgl. Steinkemper 1974). Klassische Bürokraten operieren dabei eher auf der Basis eines monistischen Verständnisses des öffentlichen Interesses, indem sie sich um die Schaffung objektiver Standards für technische Praktikabilität, Recht und Gerechtigkeit bemühen und davon ausgehen, dass Probleme vor allem sachlich zu lösen seien. Ihr Verhältnis zu den Institutionen politischer Macht wie Parlamenten, Parteien und Verbänden ist eher von Misstrauen bis hin zur Ablehnung geprägt. Dagegen erachten politische Bürokraten den politischen Einfluss auf Entscheidungsprozesse als legitim und akzeptieren die Notwendigkeit politischer Kompromisse auch jenseits von sachlichen Notwendigkeiten. Sie verhalten sich zu den Parteien eher affirmativ, vielfach sind sie sogar Mitglied einer Partei.

In der schon erwähnten grundlegenden international vergleichenden Untersuchung zu den politischen Einstellungen der Ministerialbeamten in westlichen Ländern fanden Robert Putnam u. a. (Putnam 1976, Aberbach/Putnam/Rockman 1981) schon Mitte der siebziger Jahre, dass $3 / 5$ der damals befragten Ministerialbeamten in Deutschland die politische Seite ihrer Arbeit eher positiv beurteilen (Putnam 1976, S. 39). Dies wurde von ihnen mit einiger Überraschung konstatiert, galt doch der deutsche Beamte als der klassische Bürokrat der Gegenwart. Ihre Ergebnisse zeigten jedoch schon damals eine überdurchschnittliche Aufgeschlossenheit gegenüber den politischen Anforderungen des demokratischen Prozesses. Als einen wesentlichen Erklärungsfaktor sahen die Autoren das Alter der Befragten an. Je jünger, desto weniger neigten diese dem Typ des klassischen Bürokraten zu. Gleichzeitig machten sie darauf aufmerksam, dass in der Realität die Typen nicht dichotomisch auftreten, sondern sich eher auf einem Kontinuum verteilen.

Die Studie zeigte auch, dass höhere Beamte in Bundesministerien sogar stärker als in anderen westlichen Demokratien tief in den Prozess der Politikgestaltung eingebunden waren: Sie entwickeln nicht nur Gesetzesentwürfe und Politikinhalte, sondern spielen auch eine herausragende Rolle bei der innerstaatlichen Koordinierung sowie bei Verhandlungen und Abstimmungen mit anderen Regierungsebenen und externen Akteuren wie Interessengruppen (siehe auch Mayntz/Scharpf 1975). Eine Replikation in der zweiten Hälfte der achtziger Jahre (Mayntz und Derlien 1989) sowie neuere Studien (Ebinger/Lux u. a. 2018a) bestätigen diese Befunde und unterstreichen, dass die meisten leitenden Beamten in den Bundesministerien die politische Seite ihrer Arbeit schätzen und bei der Erfüllung ihrer Aufgaben politische Überlegungen einbeziehen. Die Schwerpunktsetzung der Bundesebene auf Politikformulierung spiegelt sich somit in einer hohen funktionalen Politisierung 
der Beamten in den Bundesministerien wider. Diese funktionale Politisierung ist in Ministerien höher als in Bundesämtern, und für Spitzenpositionen höher als für niedrigere hierarchische Ränge (Ebinger/Schmitt 2010, Ebinger/Lux u. a. 2018).

Das Konzept der funktionalen Politisierung ist eng mit dem Konzept des „political craft“ verbunden, das Klaus Goetz (1997) als typisch für die deutsche Ministerialbürokratie herausgearbeitet hat. Er definiert dieses politische Handwerk als

"the ability to assess the likely political implications and ramifications of policy
proposals; to consider a specific issue within the broader context of the government's
programme; to anticipate and, where necessary, influence or even manipulate the
reactions of other actors in the policy-making process (...); and to design processes
that maximise the chances for the realisation of ministers' substantive objectives. To
do all this, senior officials need to be able to draw on personal networks of information
and communication that extend beyond their own ministry (...)" (Goetz 1997, S.754).

Politisches Handwerk umfasst also nicht nur die Fähigkeit, politische Aspekte zu berücksichtigen, sondern auch die Bereitschaft und Fähigkeit, sich aktiv am politischen Prozess zu beteiligen, den Minister zu beraten und ihm wenn notwendig zu widersprechen, um die Chance auf Zielerreichung zu erhöhen (Veit/Fromm/Ebinger 2018, Ebinger u. a. 2019) und sich in der Durchsetzung der Ziele auf Netzwerke des jeweiligen Politikbereichs zu stützen (Policy Networks).

Empirische Untersuchungen zum beruflichen Werdegang von höheren Beamten in Bundesministerien haben wiederholt gezeigt, dass viele von ihnen Berufserfahrungen in politiknahen Positionen des öffentlichen Dienstes, z. B. als persönliche Assistenten von Ministern oder Staatssekretären, im Bundeskanzleramt oder als Fraktionsmitarbeiter im Parlament sammeln, oft während sie von ihrer Tätigkeit im Ministerium beurlaubt waren (Veit/Scholz 2016). Alle diese Positionen sind nicht nur geeignet, sich „politisches Handwerk“ anzueignen, sondern können auch eine parteipolitische Verbundenheit widerspiegeln.

Ein wichtiges und prägendes Merkmal des deutschen Systems ist, dass alle Beamten, von der niedrigsten bis zur höchsten Ebene, Mitglieder politischer Parteien sein können und sehr oft sind. Dies ist in den meisten europäischen Ländern anders. Die Zulassung der Parteimitgliedschaft (auch für Soldaten) ist eine der vielen Lehren, die die „Gründerväter“ der Bundesrepublik aus den Erfahrungen des Untergangs der Weimarer Republik und des Aufstiegs und Erfolgs von Nazi-Deutschland gezogen haben. Beamte sollten lieber ihre politischen Präferenzen offenlegen als sich hinter einer vermeintlichen politischen Neutralität zu verstecken. Dies bedeutet nicht, dass alle oder auch nur die meisten Beamten Mitglied einer politischen Partei sind, aber dass die Mitgliedschaft in einer Partei eine legitime und respektable Offenlegung der eigenen politischen Ansichten ist (Jann/Veit 2015). Dabei wird von Beamten 
eine gewisse parteipolitische Zurückhaltung erwartet, und das Engagement für radikale Parteien, auch wenn sie nicht vom Verfassungsgericht verboten sind, kann zur Entfernung aus dem öffentlichen Dienst führen.

Die starke funktionale Politisierung und Einbindung von Beamten der Bundesministerien in die Formulierung und Ausgestaltung von Politikinhalten zeigt sich in einem besonderen deutschen Rechtskonstrukt, den „politischen Beamten“. Nach $\$ 54$ des Bundesbeamtengesetzes sind die beiden höchsten hierarchischen Ränge in Bundesministerien, also beamtete Staatssekretäre und Abteilungsleiter, politische Beamte. Als solche können sie jederzeit ohne Angabe von Gründen in den einstweiligen Ruhestand versetzt werden, wobei sie ihre erworbenen Pensionsansprüche behalten und jederzeit zurückgerufen werden können. Der Grundgedanke ist, dass Minister die Möglichkeit haben sollten, als ihre wichtigsten Beamten und Berater Personen ihres Vertrauens zu wählen, und wenn dieses Vertrauen - aus welchen Gründen auch immer - nicht mehr besteht, sie problemlos zu ersetzen.

Die Institution des „politischen Beamten“ und die Tradition der „politischen Pensionierung" in Deutschland reichen bis in die Mitte des 19. Jahrhunderts zurück, als in Preußen das „Lebenszeitprinzip“ für Beamte eingeführt wurde (Kugele 1976). Danach konnten Beamte nur noch dann entlassen werden, wenn sie eine disziplinarische Straftat begingen, und dies wiederum warf die Frage auf, wie ihre Macht eingeschränkt werden und wie insbesondere politische Übereinstimmung zwischen dem Monarchen und den Spitzenbeamten sichergestellt werden konnten. Deshalb wurde 1849 (als nicht wenige Beamte Sympathien für die gescheiterte Revolution von 1848 gezeigt hatten) in Preußen eine Verordnung eingeführt, die erstmals eine Aufzählung von Führungspositionen innerhalb der staatlichen Verwaltung enthielt, deren Inhaber vom König jederzeit vor-übergehend in den Ruhestand versetzt werden konnten. In den folgenden Jahrzehnten wurde dann in vielen deutschen Ländern und ab 1871 auch auf nationaler Ebene die Position dieser „politischen Beamten“ eingeführt.

Zurzeit gibt es neben der Bundeskanzlerin 15 Bundesminister und 35 parlamentarische Staatssekretäre, also insgesamt 50 Exekutivpolitiker in der Bundesregierung, und etwa 130 politische Beamte ( 25 beamtete Staatssekretäre, etwa 100 Abteilungsleiter und einige Leiter von nachgeordneten Bundesbehörden, insgesamt weit weniger als $1 \%$ aller höheren Beamten auf Bundesebene). Alle anderen Beamten in den Bundesministerien, d. h. Unterabteilungsleiter, Referatsleiter und alle unteren Dienstgrade sind Laufbahnbeamte in Planstellen. Dies bedeutet jedoch nicht, dass sie keine politisierten Funktionen erfüllen oder keine Parteizugehörigkeit haben.

Der Aufstieg in die Spitzenpositionen der Bundesministerien hängt daher sowohl von fachlicher Kompetenz als auch von parteipolitischer Bindung und Loyalität ab. Politische Beamte sind meist, aber nicht notwendigerweise, Mitglieder derselben 
politischen Partei wie ihre Minister (Fleischer 2016). Die meisten von ihnen, aber längst nicht alle, werden nach einem Regierungswechsel ersetzt. Allerdings schützt auch die „richtige" Parteizugehörigkeit nur sehr bedingt bei einem Ministerwechsel. Selbst bei gleicher Parteizugehörigkeit übernehmen nur wenige Minister politische Beamte ihrer Vorgänger in nennenswerter Zahl (Ebinger/Lux u. a. 2018). Der Anteil von Parteimitgliedern und Beamten mit klaren parteipolitischen Loyalitäten unter den Spitzenbeamten ist hoch, selbst unter den „unpolitischen“ Leitern von Unterabteilungen und Referaten (Bach/Veit 2018; Ebinger/Lux u. a. 2018). Alles dies deutet auf eine gewisse Relevanz von Ämterpatronage hin. Parteipolitisierung der Ministerialbürokratie hat in Deutschland allerdings gerade nicht die Form der Dominanz einer Partei, sondern bedeutet Parteienkonkurrenz, die Mitglieder aller koalitionsfähigen Parteien umfasst (Veit 2015). Eine Parteimitgliedschaft ist für Spitzenkarrieren im deutschen öffentlichen Dienst sicherlich nicht ohne Bedeutung. Aber während Spitzenbeamte für ihre Karriere auf politische Unterstützung angewiesen sein können, gilt dies auch umgekehrt. Minister sind ebenso oder vielleicht sogar noch mehr von der Unterstützung, der Loyalität und noch mehr von der Professionalität, dem Dienst- und Fachwissen ihrer Spitzenbeamten abhängig.

Politische Beamte fungieren daher als Bindeglied zwischen der professionellen Bürokratie und der politischen Führung und tragen dazu bei, gegenseitiges Verständnis und Vertrauen zu schaffen und Missverständnisse und Misstrauen zwischen beiden Sphären abzuschwächen. Die typischen Schuldzuweisungen zwischen Politikern und Beamten oder auch „a government of strangers“ (Heclo 2011) sind in Deutschland eher ungewöhnlich. Das Verhältnis zwischen Politik und Verwaltung, zwischen gewählten Politikern und ernannten Beamten, ist auch in Deutschland nie spannungs- und konfliktfrei, aber insgesamt scheint der politisierte öffentliche Dienst, sowohl in funktionaler als auch in parteipolitischer Hinsicht, zu weniger Konflikten, Missverständnissen und Schuldzuweisungen zu führen, als in anderen demokratischen Ländern. Spitzenbeamte in Deutschland brauchen sowohl fachliche Expertise als auch politisches Handwerk, sie geben nicht vor, unpolitisch und neutral zu sein, und die informierte Öffentlichkeit weiß in der Regel, woher ihre Spitzenbeamten kommen und wofür sie stehen. Diese politische Orientierung beeinträchtigt ihre Rolle als Beamte keineswegs, denn die Loyalität zu allen demokratisch gewählten Führern kann als selbstverständlich vorausgesetzt werden.

Trotz der wachsenden Bedeutung des „politischen Handwerks" sind die Karrieren von Politikern und Beamten in den Bundesministerien immer noch traditionell klar getrennt: Spitzenbeamte kommen in der Regel nicht aus einer Karriere im Parlament oder als Minister, und Minister waren i.d. R. keine Spitzenbeamte, auch wenn es in letzter Zeit einige bekannte Ausnahmen gibt (z. B. Steinmeier, Steinbrück, de Maizière). 
Karrieren in Bundesministerien sind - ähnlich wie in anderen Bereichen der öffentlichen Verwaltung in Deutschland - immer noch durch eine geringe inter- und intrasektorale Mobilität gekennzeichnet, d. h. Ministerialbeamte verbringen in den meisten Fällen ihre gesamte Laufbahn innerhalb eines Ministeriums. Neben der hohen Kontinuität der Karriereverläufe sind in den letzten Jahrzehnten aber auch Veränderungen zu beobachten. Zum einen ist die typische „reine Beamtenlaufbahn“, bei der eine Person direkt nach dem Hochschulabschluss in den öffentlichen Dienst eintritt und dort bis zur Pensionierung verbleibt, nicht mehr die Regel. Neueste Zahlen zeigen, dass weniger als ein Fünftel der beamteten Staatssekretäre noch zu dieser Gruppe gehören, alle anderen verfügen über Erfahrungen außerhalb der öffentlichen Verwaltung, oft in der Wissenschaft, der Justiz oder einer politischen Funktion (Veit/Scholz 2016). Unter den Abteilungsleitern sind „reine Beamtenlaufbahnen" häufiger, aber auch hier haben in den letzten Jahren gemischte Laufbahnen an Bedeutung gewonnen. Zum anderen hat sich das ehemalige "Juristenmonopol“ abgeschwächt. Während 1954 mehr als drei Viertel der Spitzenpositionen in der Bonner Verwaltung mit Juristen besetzt waren, sank der Anteil in den 2000er Jahren auf rund $50 \%$ (Veit/Scholz 2016, Ebinger/Lux u. a. 2018).

Zusammengefasst sind Politik und Verwaltung in den Bundesministerien in Deutschland durch enge Zusammenarbeit in der Politikgestaltung und einen hohen Grad an funktionaler- und Parteipolitisierung der Spitzenbürokraten einerseits, aber deutlich unterschiedliche Karrieremuster von Politikern und Bürokraten andererseits gekennzeichnet. Die wachsende Bedeutung der beruflichen Erfahrungen von Ministerialbeamten im politischen Bereich scheint jedoch auf Veränderungen hinzuweisen. Bemerkenswerterweise sind diese auf der Ebene der Länder weiter fortgeschritten, wie im nächsten Abschnitt deutlich wird.

\subsubsection{Ministerialverwaltung der Bundesländer}

Die Anzahl „Politischer Beamter“ in den Ländern ist geringer als auf Bundesebene, denn auch die Ministerien sind kleiner und haben weniger politische Aufgaben, aber in allen Bundesländern (außer Bayern) ist der oberste Beamte jedes Ministeriums (der beamtete Staatssekretär, der in Baden-Württemberg Ministerialdirektor genannt wird) ein politischer Beamter, der - ähnlich wie beim Bund - jederzeit in den einstweiligen Ruhestand versetzt werden kann. Abteilungsleiter in Landesministerien und alle Beamten der unteren Dienstgrade sind Laufbahnbeamte auf Lebenszeit, aber z. B. auch Polizeipräsidenten oder Regierungspräsidenten sind häufig politische Beamte. Insgesamt gibt es auch in den größten Bundesländern nicht mehr als zwanzig politische Beamte. Wie auf Bundesebene werden auch die Staatssekretäre in den Landesministerien in der Regel aus dem öffentlichen Dienst rekrutiert, d. h. von allen zwischen 2000 und 2018 ernannten Staatssekretären hatten 
$85 \%$ eine mindestens einjährige Berufserfahrung, etwa die Hälfte hatte mehr als zehn Jahre Erfahrung im öffentlichen Dienst.

Im Hinblick auf die Politisierung in den Landesministerien ist der Anteil der Parteimitglieder hoch (ähnlich wie auf Bundesebene). Allerdings haben die Staatssekretäre sehr häufig, d.h. in mehr als zwei Drittel aller Fälle, in früheren Karrierestufen Berufserfahrung in der Politik erworben (sei es als gewählte Politiker, als Parteiprofis oder als Beamte mit Erfahrung in politiknahen Ämtern wie z.B. als persönliche Assistenten eines Ministers). Andererseits verfügen aber auch viele Minister (etwa ein Drittel) über Berufserfahrung im öffentlichen Dienst. Dies deutet darauf hin, dass die politischen und administrativen Laufbahnen auf Länderebene nicht mehr so strikt getrennt sind wie auf Bundesebene. Fast jeder fünfte politische Beamte (18\%) war vor seiner Ernennung zum politischen Beamten hauptberuflich als Politiker (Abgeordneter) tätig, und auch politische Erfahrung auf kommunaler Ebene (etwa als gewählter Stadtrat) ist weit verbreitet, nimmt allerdings im Laufe der Zeit ab. Während Anfang der 2000er Jahre $38 \%$ der beamteten Staatssekretäre in den deutschen Bundesländern einen solchen Hintergrund hatten, ist dieser Anteil in den Jahren 2015-2018 auf weniger als ein Viertel gesunken. Insgesamt nimmt aber die Relevanz von Erfahrungen als Parteifunktionär oder in politiknahen Ämtern im Laufe der Zeit erheblich zu. Diese zunehmende Vermischung politischer und administrativer Karrieren verdeutlicht die offensichtlich zunehmende Bedeutung des "politischen Handwerks" für administrative Spitzenpositionen, gerade auch auf Landesebene (die Zahlen basieren auf einer aktuellen Untersuchung von Sylvia Veit, siehe Jann/Veit 2020).

\subsubsection{Politik und Verwaltung auf kommunaler Ebene}

\subsubsection{Institutionelle Ausgangslage}

Betrachtet man die formale Ausgestaltung des Verhältnisses von Politik und Verwaltung in den Gemeindeordnungen, so zeigt sich, dass die klassische Gewaltenteilung für die kommunale Ebene ohnehin nicht gilt. Die kommunale Vertretungskörperschaft ist in der deutschen Kommunaltradition ein Verwaltungsorgan, damit Teil der kommunalen Selbstverwaltung und der Exekutive zuzuordnen, und kein Parlament im eigentlichen Sinne, obwohl es viele Übereinstimmungen mit sonstigen Parlamenten gibt. Eine klare Trennung zwischen Politik und Verwaltung ist nicht erkennbar und auch nicht beabsichtigt. So wurde auch in der alten GO NW davon ausgegangen, 
dass die Gemeindevertretung das oberste Verwaltungsorgan ${ }^{73}$ ist, also verwaltet ( $\$ 27$ GO NW: „Träger der Gemeindeverwaltung“), also auch, dass die Verwaltung Politik macht, indem sie die Entscheidungen in der Gemeindevertretung vorbereitet $(\$ 47 \mathrm{GO} N W)$. Entscheidend für den fehlenden Status der Kommunalvertretung als Parlament ist nach Ansicht einiger Autoren, dass den Gemeinden keine eigenständige Gesetzgebungskompetenz zukommt, sie also über keine staatliche Hoheitsmacht verfügen. Im Unterschied zu Satzungen, in denen das Ortsrecht festgehalten wird, können Gesetze nur von den Landesparlamenten und vom Bundestag erlassen werden. Beschlüsse der Gemeindevertretung können im Aufsichtswege beanstandet, aufgehoben oder sogar ersetzt werden $(\$ 108,109$ GO NW), das Innenministerium kann einen Beauftragen zur Erfüllung einzelner Aufgaben einsetzen ( $\$ 110$ GO NW) und der Rat kann durch Beschluss der Landesregierung sogar aufgelöst werden (\$111 GO NW), so dass, wie Forsthoff schon früh ausführte, von einem originären Parlamentsrecht nicht gesprochen werden kann (1973, S. 551f.). Zudem haben die Gemeinden nur sehr begrenzt Einfluss auf die eigenen Einnahmemöglichkeiten.

Trotz dieser Einschränkungen hat sich in der kommunalen Praxis zumindest in den großen Städten kommunale Selbstverwaltung zu einer modernen lokalen Demokratie entwickelt (Wollmann 1998c). Insbesondere in den größeren Kommunen haben sie sich in ihrer Arbeitsstruktur durchaus den Arbeitsstrukturen von Parlamenten angenähert. So sind auch in den Kommunalvertretungen vor allem die Ausschüsse wichtig, in denen alle Entscheidungsvorlagen vorbesprochen werden. Zudem gibt es seit Ende der siebziger Jahre einen Ausbau „parlamentarischer Rechte“ in den Gemeinderäten und Kreistagen durch die Schaffung von Minderheitsrechten von Fraktionen bezüglich der Einberufung des Rates, der Durchsetzung von Tagesordnungspunkten, des Akteneinsichtsrechts und der Forderung nach namentlicher und geheimer Abstimmung. Damit wird die Oppositionsrolle in der kommunalen Vertretungskörperschaft rechtlich deutlich aufgewertet. Zudem gibt es überall Regelungen zum Verdienstausfall und Aufwandsentschädigungen, die aber von Kommune zu Kommune anders gehandhabt werden.

Die kommunalen Vertretungskörperschaften werden zudem wie ein Parlament gewählt, die Mitglieder schließen sich zu Fraktionen zusammen, haben das Recht, kommunale Satzungen zu beschließen und fühlen sich als Parlamentarier. Von den klassischen Parlamentsrechten verfügt man somit über die Möglichkeit der politi-

73 In Baden-Württemberg gibt es dagegen schon immer zwei Organe, den Gemeinderat als Hauptorgan und den Bürgermeister ( $\$ 23 ; 24,1$ GO BaWü). Allerdings kam in NRW dem Gemeindedirektor aufgrund seiner Zuständigkeiten bei der Leitung und Verteilung der Geschäfte nach einem Urteil des Oberverwaltungsgerichtes von 1954 die Rechtsstellung eines Organs zu, obwohl dies zunächst nicht in der GO kodifiziert wurde. 
schen Leitungsentscheidung, über das Budgetrecht, über die Normsetzungsbefugnis sowie über die Kontrollfunktion. Zur Wahrnehmung ihrer Kontrollaufgaben ( $\$ 40, \$ 49$ GO NW) verfügen die Vertretungskörperschaften neben dem schon erwähnten Budgetrecht über ein Frage- und Antwortrecht, das umfassende Recht auf Information sowie die Möglichkeit, den Verwaltungschef abzuwählen. Wollmann sieht diesen Parlamentarisierungsprozess als Verwirklichung der ursprünglichen Grundgesetzintention an, nämlich des Art. 28, Abs. 1, Satz 2, der vorsieht, dass „das Volk (...) in den Ländern, Kreisen und Gemeinden eine Volksvertretung" (Wollmann 1998a, S. 406, 1998c, S. 50ff.) haben müsse. Auch Ott spricht hier von einem Parlamentscharakter der Gemeindevertretung (Ott 1994).

Die Kommunalpolitiker arbeiten ehrenamtlich, d.h., sie erhalten nur Aufwandsentschädigungen. Allerdings nehmen die Komplexität und die zu verarbeitenden Informationen mit der Gemeindegröße stark zu, sodass zumindest in den 79 deutschen Großstädten mit mehr als 100.000 Einwohnern häufig die Grenzen der Ehrenamtlichkeit erreicht sind. Eine repräsentative Studie zur Situation des kommunalen Ehrenamtes aus dem Jahre 2017 in NRW zeigt, dass der mittlere Zeitaufwand in den Städten 32,5 Stunden im Monat beträgt (Kreise: 29,7 Stunden). Erwartungsgemäß sind es im Vergleich die Fraktionsvorsitzenden, die die meiste Zeit in ihre Tätigkeit investieren (43,9 Stunden je Monat; Kreise 40,8; vgl. Bogumil/ Gehne/Garske 2017, S. 62). ${ }^{74}$ In den einwohnerstärksten Städten Nordrhein-Westfalens fällt der Zeitaufwand bei Fraktionsvorsitzenden mit 56,4 Stunden noch deutlich höher aus. Die Ratsmitglieder in den Großstädten erhalten im Jahr 2006 eine durchschnittliche Gesamtaufwandsentschädigung aus Pauschale und/oder Sitzungsgeld von monatlich 521 Euro (vgl. Reiser 2006). Während es in Erfurt nur 187 Euro pro Monat sind, erhalten die die Stuttgarter Stadträte 2.460 Euro pro Monat (zum Vergleich Hamburg 2.456 Euro, Bundestag 7.009 Euro zum gleichen Zeitpunkt). Zudem wird den Fraktionen ermöglicht, Fraktionspersonal einzustellen. Durchschnittlich erhält eine Fraktion in den Großstädten pro Fraktionsmitglied Zuwendungen in Höhe von 9.289 Euro pro Jahr. Auch hier schwanken die Beiträge beträchtlich, zwischen 30.612 Euro in Frankfurt und 612 Euro in Heilbronn (zum Vergleich Hamburg: 26.682, Bundestag 88.731 Euro, vgl. Reiser 2006).

Die Größe der Gemeindevertretung hängt von der Gemeindegröße ab. Dabei gibt es bezüglich der Anzahl der Sitze zwischen den Bundesländern leichte Abwei-

74 In einer der größten Befragungen von Rats- und Kreistagsmitgliedern in der Bundesrepublik Deutschland wurden im Auftrag des Landes Nordrhein-Westfalen $10 \%$ aller Ratsmitglieder befragt, das waren 2.283 kommunale Mandatsträgerinnen und Mandatsträger aus 44 Städten und Gemeinden und sechs Landkreisen. Rund 61 \% von ihnen nutzten die Möglichkeit zur Rückantwort (vgl. ebd., S. 5). 
chungen, sodass z. B. in NRW die Räte prinzipiell größer sind. Hat eine Stadt z. B. etwas über 400.000 Einwohner, gibt es in Baden-Württemberg und Bayern 60 Sitze und in NRW 74. Bei Kommunen mit einer Größe von 5.000 Einwohnern sind es entsprechend 18, 20 und 26 Gemeinderatssitze. Insgesamt gibt es in NRW 15.818 Kommunalmandate. Aufgrund der größeren Anzahl an Kommunen sind es in Baden-Württemberg 18.216 und in Bayern 32.576 (vgl. Bogumil/Holtkamp 2013, S. 42).

Die Befragung aus dem Jahr 2017 in NRW ergab zudem, dass der typische kommunale Mandatsträger männlich ist, über 55 Jahre alt und hoch gebildet. Damit unterscheiden sie sich nicht wesentlich von anderen Mandatsträgern in Deutschland. Zwar fällt keine Alterskohorte komplett aus, doch sind es vor allem Schüler und Studenten, Berufseinsteiger und Mandatsträger im Lebensalter zwischen 30 und 40, die im Vergleich zur Bevölkerung erheblich unterrepräsentiert sind. Der Frauenanteil ist mit $27 \%$ in den Städten und $29 \%$ in den Kreisen - gemessen an dem Bevölkerungsdurchschnitt - immer noch gering. Zweidrittel der Mandatsträger haben die Fachhochschulreife oder die Hochschulreife erworben, während der Anteil in der Bevölkerung bezogen auf die 20-65-Jährigen bei $33 \%$ liegt, so dass von einer deutlichen Akademisierung der Vertretungskörperschaft auszugehen ist (vgl. Bogumil/Gehne/Garske 2017, S.37).

Zusammenfassend zeigt sich, dass die Kommunen nach dem deutschen Kommunalrecht den Status einer besonderen Form politischer Verwaltung innehaben, der durch seine Unklarheit mit für die Konflikte zwischen eher verwaltungs- und sachorientierten sowie parteienstaatlichen Konzeptionen kommunaler Selbstverwaltung verantwortlich ist. Dabei gibt es zwischen den einzelnen Bundesländern trotz der in Kapitel 3.5.3 geschilderten Angleichung der Kommunalverfassungen deutliche Unterschiede in der Ausgestaltung des institutionellen Rahmens, die sich zwischen den Extremen eher konkurrenzdemokratisch und eher konkordanzdemokratisch ausgerichteten Kommunalverfassungen bewegen (vgl. Kap. 3.5.3).

\subsubsection{Empirische Erkenntnisse}

Neben dieser normativen Diskussion, wie eigentlich die kommunale Selbstverwaltung einzuschätzen sei, gibt es auch einige empirische Erkenntnisse zum Verhältnis von Politik und Verwaltung auf lokaler Ebene. Seit Anfang der neunziger Jahre lassen sich grob fünf wesentliche Trends unterscheiden, die das alte Verhältnis von Politik, Verwaltung und Bürgern zunehmend berühren (vgl. Bogumil 2001):

- zunehmende Haushaltskonsolidierungsanstrengungen,

- Liberalisierungs- und Privatisierungsbestrebungen vor allem im Bereich kommunaler Daseinsvorsorge, 
- die flächendeckende Einführung von direkt-demokratischen Elementen (Direktwahl des hauptamtlichen Bürgermeisters, Bürgerbegehren/-entscheide),

- die zunehmende Bedeutung kooperativer Demokratieelemente (z.B. durch Lokale-Agenda-Prozesse, Mediationsverfahren) sowie

- die umfassenden Bemühungen zur Verwaltungsmodernisierung nach dem Neuen Steuerungsmodell (NSM) im Rahmen der Public-Management-Reformen.

Hierbei handelt es sich um recht unterschiedliche Entwicklungen, die zudem an verschiedenen Stellen im kommunalen Entscheidungsprozess ansetzen. Wie wirken sich die Modernisierungsimpulse nun im kommunalen Kräftedreieck zwischen Rat, Verwaltungsspitze und Bürgerschaft aus? Das folgende Schaubild zeigt eine sehr verdichtete Zusammenfassung bezogen auf die Einflusschancen der verschiedenen Akteure (vgl. ausführlich Bogumil/Holtkamp/Kißler 2004).

\begin{tabular}{|c|c|c|c|c|c|c|}
\hline & $\begin{array}{l}\text { Haushalts- } \\
\text { krise }\end{array}$ & $\begin{array}{l}\text { Privatisie- } \\
\quad \text { rung }\end{array}$ & $\begin{array}{c}\text { Direkt- } \\
\text { wahl }\end{array}$ & $\begin{array}{c}\text { Bürger } \\
\text { begehren }\end{array}$ & $\begin{array}{l}\text { Kooperative } \\
\text { Demokratie }\end{array}$ & NSM \\
\hline Bürgermeister & 0 & - & + & - & + & 0 \\
\hline Verwaltung & + & - & 0 & - & + & + \\
\hline Vertretung ${ }^{75}$ & - & - & - & - & - & 0 \\
\hline Bürger & - & - & + & + & + & + \\
\hline
\end{tabular}

Abb. 53 Wirkungen lokaler Modernisierungstrends auf die Einflusschancen der Akteure

Quelle: Bogumil/Holtkamp 2006

Spitzt man die Ergebnisse zu, so zeigt sich zum einen, dass die gewählte Kommunalvertretung der absolute Verlierer der Entwicklungen ist und immer geringere Handlungsspielräume hat. Die Haushaltskrise und zunehmende Privatisierungen schränken die traditionellen Politikgestaltungsmöglichkeiten ein, durch die Direktwahl des Bürgermeisters und durch die Einführung von kommunalen Referenden entstehen Vetopositionen sowohl für die Verwaltungsleitung als auch für die Bürger, wenn auch in unterschiedlichem Ausmaß. Aber auch diese Vetopositionen schränken den Handlungsspielraum der kommunalen Vertretungskörperschaften ein. Dies gilt in schwächerem Maße auch für kooperative Bürgerbeteiligungsmöglichkeiten, die,

75 Vor allem die Mehrheitsfraktionen sind hier in ihrem Handlungsspielraum getroffen, für die Oppositionsfraktionen ergeben sich mitunter sogar neue Handlungsspielräume, z. B. durch die Nutzung von Bürgerbegehren. 
einmal installiert, Eigendynamiken entwickeln. Allerdings hat die Vertretung durch Verteidigungsstrategien durchaus die Möglichkeit, einen Teil der Handlungsspielräume zu konservieren. Dies gilt nicht nur für die neue Arbeitsteilung zwischen Vertretung und Verwaltung im Rahmen der Verwaltungsreformen des NSM, die durchweg gegen ihren Widerstand nicht umgesetzt wurde. Auch im Rahmen der kooperativen Demokratie ist teilweise zu beobachten, dass die Kommunalvertretung zwar immer mehr Bürgerforen einrichtet, aber nur wenig Bereitschaft zeigt, hinterher auch Beteiligungsergebnisse umzusetzen, sondern eher ihre eigenen Präferenzen realisieren will. Diese Verteidigungsstrategie führt aber dazu, dass sich Partizipationsenttäuschungen bei den Bürgern verschärfen können. Weiterhin ist in manchen Bundesländern wie in Nordrhein-Westfalen zu beobachten, dass die Vertretungsmehrheiten allzu selbstbewussten Bürgermeistern über die Hauptsatzung oder das Aufbauen von starken Beigeordneten Einhalt gebieten. Nur führt dies nicht selten zu einer Politikblockade. Der Bürgermeister kann viele Dinge nicht mehr alleine voranbringen und revanchiert sich dafür mit der mangelhaften Vorbereitung und Implementation von Vertretungsbeschlüssen. Die Selbstverteidigungsstrategien der Vertretung können also nicht unbeträchtliche negative Nebenfolgen hervorbringen.

Die Verwaltungsspitze, repräsentiert durch den hauptamtlichen Bürgermeister, kann in der Summe ihr Machtpotential durchaus ausbauen. Sie wird zwar auch durch Privatisierungsmaßnahmen und die Einführung von Bürgerbegehren negativ in ihren Einflussmöglichkeiten tangiert, erhält aber vor allem durch die Direktwahl eine deutliche Stärkung im kommunalen Entscheidungssystem. Auch zeigt sich, dass die Bürgermeister in der Regel Bürgerbeteiligungsmaßnahmen sehr gut für ihre eigenen Zwecke nutzen und damit häufig die kommunalen Vertretungskörperschaften unter Druck setzen können. Ähnliches gilt für die hauptamtliche und professionelle Verwaltung, die durch ihren Informationsvorsprung und ihre vielfältigen Kontakte sowohl die Möglichkeiten des Neuen Steuerungsmodells, wie auch der kooperativen Demokratie, und nicht selten sogar der Haushaltskrise zur Durchsetzung ihrer Präferenzen nutzen kann.

Bezogen auf die Bürger gibt sich ein zwiespältiges Bild. Einerseits haben die Bürger immer mehr Möglichkeiten sich in das politische System einzubringen, andererseits wird es aufgrund der geringeren Handlungsspielräume durch Privatisierung und Haushaltskrise immer unwahrscheinlicher, dass dies Auswirkungen auf den Output des kommunalpolitischen Systems hat. Während die stärkere Bürgerorientierung der drei partizipativen Modernisierungstrends (Bürgerbegehren, Direktwahl und kooperative Demokratie) bei den Bürgern also die Erwartungen weckt, dass sie zukünftig mehr Einfluss auf die Kommunalpolitik nehmen können, reduzieren stetig abnehmende kommunale Handlungsspielräume demgegenüber die Einflussmöglichkeiten und können zu einer Enttäuschung dieser Erwartungen 
führen. Die Bürger werden so zwar immer mehr nach ihrer Meinung gefragt, aber ihre Meinung ist aufgrund geringerer Handlungsspielräume immer folgenloser. Frustration ist deshalb vorprogrammiert. Der Anspruch der anderen Modernisierungstrends, Politik(er)verdrossenheit durch Beteiligung abzubauen, könnte unter diesen Rahmenbedingungen in sein Gegenteil umschlagen.

\subsubsection{Normative Bilder der Verwaltung}

Die Rolle der öffentlichen Verwaltung im demokratischen Staat ist, wie gezeigt, keinesfalls selbstverständlich und unproblematisch. Die Verwaltung ist in Gewaltenteilungslehren und Demokratietheorien keine eigenständige Kategorie neben Judikative und Legislative, aber sie sollte und müsste es eigentlich sein, denn die bequeme Subsumptionen unter "Exekutive“ oder auch "politisch-administratives System“ (PAS) verschleiern die Problematik mehr, als dass sie diese erhellen würden (zum folgenden ausführlich Jann 1998a m. w. A., aus dem weite Teile übernommen wurden).

So argumentiert z. B. die traditionelle Verwaltungsrechtswissenschaft im Anschluss an Otto Mayer, Verwaltung sei „Tätigkeit des Staates, die nicht Gesetzgebung oder Justiz ist" (Mayer 1895, S.7), und H. Peters hat sich in einer einflussreichen Schrift „Die Verwaltung als eigenständige Staatsgewalt“ (Peters 1965) ausdrücklich mit einer möglichen Trennung von Regierung und Verwaltung auseinandergesetzt und diese Unterscheidung mit dem Argument abgelehnt, im Gewaltenteilungsschema gebe es keinen Platz für eine abgesonderte Regierung (Thieme 1984, S. 3). Ausgangspunkt ist jeweils die klassische Trennung des Regierungssystems in Legislative, Judikative und Exekutive, die als abschließend betrachtet wird. Für Verwaltung gibt es in dieser Theorietradition keinen Platz.

In einer klassisch-konservativen Staatstheorie, bei der Demokratie ohnehin nur „staatsformabhängiges Beiwerk“ ist (Krüger 1966) und einzig der Staat, repräsentiert durch seinen hoheitlichen Apparat, sich dadurch auszeichnet, dass er allein das Gemeinwohl erkennen und durchsetzen kann (ausführlich Böhret/Jann/Kronenwett 1979, S. 256ff.), ist diese Einheit von Regierung und Verwaltung kein Problem, sie ist sogar Kern dieses Staatsverständnisses. Die Exekutive ist der eigentliche Staat und die Bürokratie die eigentliche Exekutive. Das Beamtentum ist der „allgemeine Stand“, der „die allgemeinen Interessen des gesellschaftlichen Zustandes zu seinem Geschäfte" hat (Hegel, Rechtsphilosophie $\$ \$ 205,303)$.

Diese Theorie lässt sich im demokratischen Verfassungsstaat nicht mehr überzeugend vertreten. Die funktionelle Differenzierung, insbesondere das Entstehen hochgradig organisierter funktioneller Teilsysteme der Gesellschaft, die mit den Stichworten Spezialisierung, Differenzierung, Interdependenz und nicht zuletzt 
Enthierarchisierung charakterisiert werden kann (Mayntz 1996), hat eine erhebliche territoriale und funktionale Differenzierung des öffentlichen Sektors erzeugt. Dabei erstrecken sich die gesellschaftlichen Widersprüche in den Staat selbst und verringern seine Kohärenz. Je mehr staatliche Steuerung, desto mehr Zielkonflikte. Unterschiedliche Verwaltungszweige verfolgen - wissentlich oder absichtslos unvereinbare Zwecke und verursachen Folgewirkungen in anderen Ressorts. Der Bedarf an Koordination übersteigt zunehmend die Kapazitäten des politischen Systems, auf keinen Fall kann mehr von einer einfachen politischen Steuerung der Verwaltung ausgegangen werden.

Offenbar macht es auch keinen Sinn mehr, von einer einheitlichen öffentlichen Verwaltung zu sprechen. Das Bild von der öffentlichen Verwaltung als hierarchischer, einfach zu steuernder Pyramide war vermutlich nie realistisch und ist heute auf jeden Fall grob irreführend. Die öffentliche Verwaltung ist viel zu spezialisiert, differenziert und fragmentiert, als dass sie diesem Bild noch entspräche. Statt einer festen Pyramide entsteht eher das Bild eines vielfältig verknüpften und sich weitgehend selbst tragenden Netzes. Es erscheint daher sinnvoller, vom „öffentlichen Sektor“ zu sprechen, als von der "öffentlichen Verwaltung“ oder vom „öffentlichen Dienst“. Insbesondere Fritz W. Scharpf hat immer wieder darauf hingewiesen, dass der öffentliche Sektor sich so stark differenziert hat und so sehr mit anderen gesellschaftlichen Sektoren verflochten ist, dass die mit der Steuerungs-Metapher fast immer unterstellten Voraussetzungen einer aus einheitlicher Absicht hervorgebrachten Willensbildung und Willensdurchsetzung des ,Staates' nicht mehr gegeben sind, falls es sie je gab.

Dass sich aus dieser Erkenntnis erhebliche Probleme der politischen Steuerung der Verwaltung und ihrer Legitimation ergeben, liegt auf der Hand. Demokratietheoretisch ist das klassische Modell von der gesetzesgebundenen, streng bürokratisch und hierarchisch organisierten und handelnden Verwaltung viel unproblematischer, als das der flexibel handelnden, mit ihrer gesellschaftlichen Klientel verflochtenen, weitgehend autonomen Verwaltung.

Im Folgenden soll es darum gehen, diese Problematik in unterschiedlichen, mehr oder weniger expliziten Sichtweisen der Rolle der öffentlichen Verwaltung deutlich zu machen, zu systematisieren und dabei pointiert zusammenzufassen. In diesem Zusammenhang werden vier normative Bilder der öffentlichen Verwaltung im Regierungssystem unterschieden, deren jeweilige Herausbildung unterschiedlichen Entwicklungsstufen des demokratischen Staates zugeordnet werden kann, die aber auch heute noch alle in bestimmten Bereichen der Verwaltung relevant sind. Die Unterscheidung dieser Modelle geht zurück auf eine umfassende empirische Untersuchung des dänischen öffentlichen Sektors, in der 120 öffentliche Organisationen analysiert und in einer Datenbank mit über 1000 Variablen beschrieben wurden (siehe Beck Jørgensen 1993, Antonsen/Jørgensen 1995). 


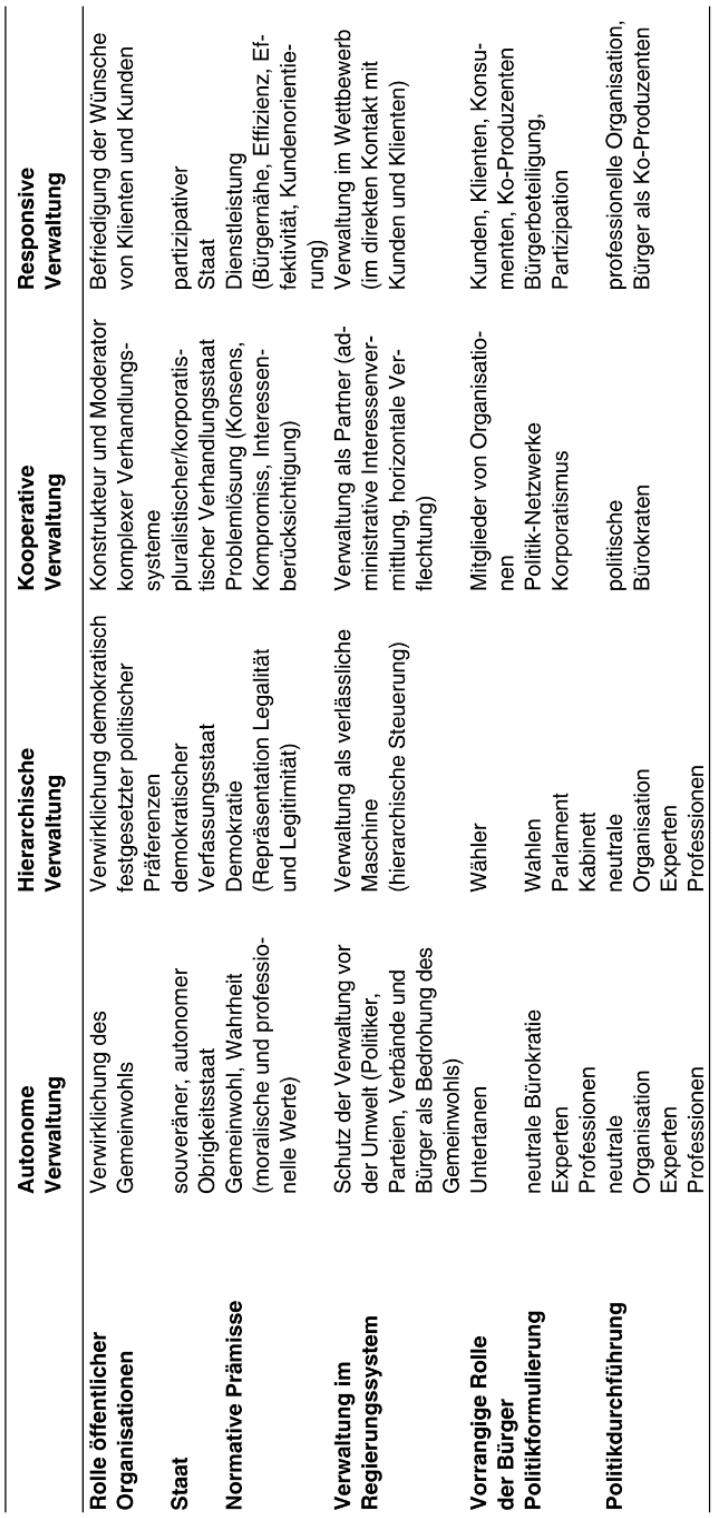

Abb. 54 Normative Bilder der Verwaltung

Quelle: eigene Darstellung 
„Öffentliche Verwaltung“ wird dabei zunächst ganz einfach institutionell verstanden, im Gegensatz z. B. zu der oben vorgenommenen personellen Unterscheidung zwischen „Bürokraten“ (Beamten, Angehörigen des öffentlichen Dienstes) und „Politikern“ (nicht ernannten, sondern gewählten Funktionären). Ganz allgemein sollen unter öffentliche Verwaltung alle Organisationen subsumiert werden, die - im deutschen Rechtssystem - öffentlich-rechtlich organisiert sind und/oder überwiegend durch in öffentlichen Haushaltsplänen festgelegte Finanzen finanziert werden (also z. B. auch öffentliche Theater, Eigenbetriebe, Universitäten oder Schulen, öffentliche Stiftungen, Körperschaften und Anstalten). Ausgangspunkt der folgenden Überlegungen ist daher die formal definierte „öffentliche Organisation", die in vier unterschiedlichen normativen Bildern der öffentlichen Verwaltung typologisch unterschieden wird, nämlich als

- autonome Verwaltung

- hierarchische Verwaltung

- kooperative Verwaltung und

- responsive Verwaltung.

\subsubsection{Normative Grundlagen}

Ausgangspunkt traditioneller Vorstellungen der Verwaltung ist die „autonome Verwaltung". Die Funktion öffentlicher Organisationen besteht in diesem Idealtypus darin, das Gemeinwohl zu erkennen und zu verwirklichen. Normative Grundlage ist das a priori vorhandene Gemeinwohl, das "nur" erkannt und umgesetzt werden muss. Darin besteht die eigentliche Aufgabe des Staates, und nur er ist dazu in der Lage. Der Staat wiederum besteht aus seinen loyalen Beamten. Zentrales Merkmal dieses Beamtenkörpers sind gemeinsame moralische, ethische und professionelle Werte, die vor allem durch eine spezifische Sozialisation vermittelt werden können. Organisationsprinzip der öffentlichen Verwaltung ist daher die autonome Bürokratie, die letztendlich vor den unsachlichen und das Gemeinwohl verfälschenden Einflüssen der Umwelt geschützt werden muss. Diese feindliche Umwelt besteht insbesondere aus Interessengruppen, die gemeinwohlfeindliche Eigeninteressen verfolgen, aber auch der Politik, insbesondere den eigensüchtigen Parteien, im Endergebnis aber auch aus den egoistischen, uninformierten und vorurteilsbeladenen Bürgern. Siehe z. B. die Auffassung eines traditionellen Staatsrechtlers wie Herbert Krüger:

„Anders als für die Gesellschaft im Verhältnis zum Staat gibt es (...) für den Staat keinen spezifischen Schutz vor der Gesellschaft. Umso mehr muss daher vor allem 
Bedacht auf die Abschirmung des Staates gegenüber dem natürlichen Menschen genommen werden“ (Krüger 1966, S. 629, ausführlich Böhret/Jann/Kronenwett 1982).

Die vorrangige Rolle der Bürger ist die der Untertanen dieses paternalistischen Obrigkeitsstaates. Politikformulierung, die Festlegung öffentlicher Aufgaben, und ihre Umsetzung, ,was soll der Staat tun und wie soll er dies tun“, obliegt der neutralen Bürokratie. Es gibt keinen prinzipiellen Unterschied zwischen Politikformulierung und -durchführung, allenfalls ist dies eine Frage der Kompetenz. Die Implementation wird von weniger kompetenten Angehörigen der Verwaltung vorgenommen, daher muss sie hierarchisch überwacht und gesteuert werden.

Das nächste Bild ist das der hierarchisch organisierten Verwaltung im demokratischen Rechts- und Verfassungsstaat. Hier besteht die zentrale Funktion öffentlicher Organisationen in der Verwirklichung demokratisch definierter politischer Präferenzen. Normative Grundlage dieses Bildes ist die Demokratie, und zwar im Sinne von Dahl als Polyarchie, d.h. gekennzeichnet durch Organisations-, Koalitions-, Meinungs- und Versammlungsfreiheit, allgemeines Wahlrecht, Offenheit sämtlicher öffentlicher Ämter für alle Bürger, und vor allem das Recht, um Ämter und Unterstützung zu konkurrieren, Informationsfreiheit und alternative Angebote von Informationen, freie, gleiche, geheime und faire Wahlen und insbesondere die Existenz von Institutionen, die Politikinhalte und deren Umsetzung an Wahlen, Abstimmungen und andere Manifestationen von politischen Präferenzen binden (Dahl 1971, Schmidt 1995, Böhret/Jann/Kronenwett 1979). Dies ist das klassische Bild des demokratischen Verfassungsstaates. Das Idealbild der öffentlichen Verwaltung ist darin das der Bürokratie als verlässlicher Maschine, die all diese demokratischen Funktionen ohne Bias, „sine ira et studio“, fair, neutral und wie die Adjektive alle heißen, unterstützt. Im Gegensatz zum ersten Bild soll die Verwaltung hier alles andere als autonom sein, sie soll stattdessen vollständig demokratisch determiniert und kontrolliert werden.

Die vorrangige Rolle der Bürger ist die der Wähler, die ihre Präferenzen und Interessen über ihre gewählten Repräsentanten qua demokratisch-legitimierter Gesetzgebung und Regierung der Verwaltung vorgeben. Politikformulierung geschieht durch Wahlen, Parlamente, Kabinette, Parteien und alle anderen legitimen Möglichkeiten demokratischer Willensbildung - durchaus unterstützt von einer loyalen Bürokratie. Es gibt eine parlamentarische Steuerungs- und Legitimationskette, die entweder über Wahl-Parlament-Gesetze-Bürokratie (legislative Programmsteuerung) oder Wahl-Parlament-Regierung-Bürokratie (demokratisch-legitimierte Weisung) funktioniert. Die Politikdurchführung ist demgegenüber weiterhin der neutralen Expertise und Professionalität der Bürokratie überlassen. Dies ist das Bild der klassischen Dichotomie zwischen Politik und Verwaltung. 
Nachdem auch dieser Idealtypus sich als wirklichkeitsfremd - selbst für einen Idealtypus - erwiesen hatte, entstand das Bild der kooperativen Verwaltung (grundlegend Benz 1994, Dose 1997, Dose/Voigt 1995). Hier erweitert sich die Funktion der öffentlichen Verwaltung in Richtung eines Konstrukteurs und Moderators komplexer Verhandlungssysteme zwischen öffentlichem und privatem Sektor. Normative Prämisse ist hier die Ermöglichung adäquater Problemlösungen durch die Berücksichtigung und den Ausgleich legitimer Interessen und der internen Strukturen und Funktionen sozialer, weitgehend selbst-regulierender Subsysteme und kollektiver Akteure (Scharpf 1993, Mayntz 1996). Dies ist das Bild des hoch-differenzierten, pluralistischen und korporativen Verhandlungsstaates.

Die Bürokratie ist in diesem Bild kompetenter Verhandlungspartner in und auch Architekt von Politiknetzwerken, dabei wird nicht prinzipiell zwischen Politik und Verwaltung unterschieden. Akteur ist das politisch-administrative System. Eine zentrale Rolle der Verwaltung besteht in der administrativen Interessenvermittlung, d. h. in der Aggregation und durchaus auch Artikulation gesellschaftlicher Interessen in enger Verbindung mit gesellschaftlichen Organisationen (Lehmbruch 1987). Bürger treten dieser Verwaltung vorrangig als Mitglieder von Organisationen gegenüber. Je differenzierter und organisierter die Gesellschaft, desto handlungsfähiger sind im Prinzip corporate actors und das politisch-administrative System. Politikformulierung geschieht daher notwendigerweise in hoch-differenzierten Politiknetzwerken, unter Mitwirkung von betroffenen Verbänden, Unternehmen, Wissenschaft und natürlich auch politischen Organisationen. Die Politikdurchführung wird weiterhin einer professionellen Bürokratie überlassen, die allerdings über erhebliche Verhandlungs- und Handlungsspielräume verfügen muss. Die hierarchische Überordnung des Staates über die Gesellschaft wird zunehmend infrage gestellt.

Schließlich gibt es ein weiteres, „moderneres" Bild der öffentlichen Verwaltung, nämlich das der responsiven Dienstleistungsorganisation. Hier liegt die zentrale Aufgabe der öffentlichen Verwaltung in der Befriedigung der konkreten Wünsche und Bedürfnisse der Bürger, also ihrer Klienten und ihrer „Kunden“ (Oppen 1995, KGSt 1992). Die Verwaltung als öffentliche Organisation ist eher der Gesellschaft untergeordnet als der Politik.

Normative Prämissen sind in diesem Modell die effektive und effiziente Dienstleistung und „Kundenorientierung“. Die Präferenzen der Bürger werden nicht, wie im autonomen Modell, von der Verwaltung selbst definiert, sie werden auch nicht, wie im hierarchischen Modell, per Gesetz oder Regierung vorgegeben und auch nicht in komplexen Verhandlungssystemen festgelegt, sondern sie werden direkt gegenüber der Verwaltung artikuliert und durch direkten Austausch durchgesetzt. Das Idealbild der öffentlichen Verwaltung ist hier die Bürokratie im Wettbewerb. Die Bürger können sich ihre öffentlichen Dienstleistungen in der Konkurrenz 


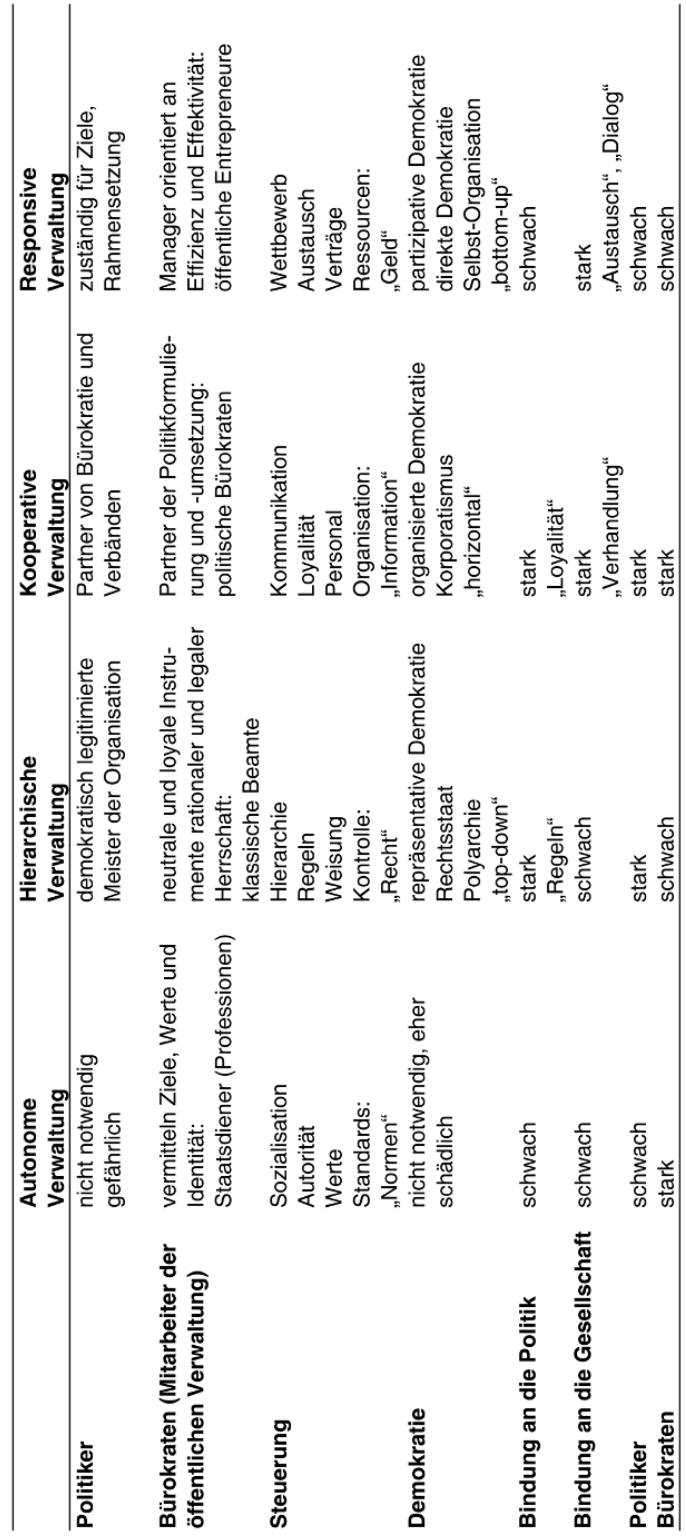

Abb. 55 Verhältnis zwischen Politikern und Bürokraten

Quelle: eigene Darstellung 
einer Vielfalt von Anbietern, seien sie privat, aus dem sog. Dritten Sektor oder auch andere öffentliche Organisationen aussuchen. Bürger sind daher vorrangig Klienten, Konsumenten, Kunden der öffentlichen Verwaltung, oder auch, in einem modernen Verständnis von Dienstleistungen, aktive Ko-Produzenten dieser Leistungen. Politikformulierung geschieht durch direkte Beteiligung und aktive Teilnahme der Bürger, Partizipation ist hier die normative Leitlinie.

Im nächsten Abschnitt soll genauer untersucht werden, wie die Realität der öffentlichen Verwaltung in Deutschland mit diesen normativen Bildern korrespondiert. Vorher ist es aber notwendig, kurz das prekäre Verhältnis zwischen Politik und Verwaltung in diesen Modellen anhand der ihnen zugeordneten Akteure, also der Politiker und Bürokraten zu spezifizieren.

\subsubsection{Politiker}

In der autonomen Verwaltung gibt es eigentlich keinen Bedarf für Politiker. Sollte es sie dennoch geben, sind sie für die Verwirklichung des Gemeinwohls eher schädlich und gefährlich. Politikern fehlt die notwendige fachliche Expertise, sie vertreten allein ihre bornierten, kurzfristigen und unsachlichen Einzelinteressen und behindern so sachlich adäquate Problemlösungen. Spätestens jetzt fällt auf, dass diese Bestimmung der Rolle von Politik und Verwaltung offenbar keineswegs historisch überholt ist. Die skizzierten Bilder vermitteln nicht nur eine historische Abfolge normativer Bilder, sondern sie existieren gleichzeitig in allen modernen, öffentlichen Organisationen. Die Vorstellung, dass Politik die Verwaltung bei der "sachgerechten“, „gemeinwohlorientierten“ Erledigung öffentlicher Aufgaben behindert, ist immer noch aktuell.

In der hierarchischen Verwaltung sind Politiker demgegenüber, zumindest im normativen Selbstverständnis, die unangefochtenen demokratisch legitimierten Meister der Bürokratie. Sie bilden die Spitze der bürokratischen Pyramide, nur durch sie werden Präferenzen und Werte für die Verwaltung selektiert. Dieses Bild entspricht in etwa Image I und II der Darstellung von Aberbach/Putnam/Rockman (siehe oben 4.4.1) und auch das Bild, das die öffentliche Verwaltung gern selbst von sich zeichnet, insbesondere wenn sie unter Kritik gerät. So werden regelmäßig gern alle Kritikpunkte an der Verwaltung mit dem Hinweis auf die Verantwortung der Politik zurückgewiesen. Allein die Politik erfindet neue staatliche Aufgaben, die bedauernswerte Verwaltung muss sie dann ausführen und dafür die Kritik einstecken.

In der kooperativen Verwaltung sind Politiker Partner der Bürokratie. In Politiknetzwerken und "issue networks" arbeiten sie mit Organisationen zusammen und sind, genau wie Politiker, bei der Artikulation und Aggregierung von Interessen unverzichtbar. Dies entspricht weitgehend Image III und IV der Aberbach/Putnam/ Rockman-Darstellung. Dieses Bild ist insbesondere anhand der Politikformulierung 
im Bereich der Ministerialbürokratie des Bundes oder der Europäischen Union empirisch hervorragend belegt.

Fraglich ist, wie die Rolle der Politiker im Rahmen der responsiven Verwaltung zu sehen ist. Das Neue Steuerungsmodell forderte apodiktisch eine neue Aufgabenteilung zwischen Politik und Verwaltung, in der die einen für die Zielsetzung und Überwachung öffentlicher Aufgabenerfüllung zuständig sein sollen, die anderen für die Umsetzung. Aber das ist im Prinzip nur eine Wiederholung ihrer normativen Rolle im hierarchischen Modell, allerdings ergänzt durch neuartige Kontrollinstrumente (Kosten- und Leistungsrechnung, Berichtswesen, Controlling etc., ausführlich Bogumil/Kißler 1997). Wenn aber deutlich geworden ist, dass diese normative Rollenbestimmung im klassischen Modell problematisch ist, warum sollte sie jetzt plötzlich als Richtschnur der responsiven Verwaltung ausreichen? Es ist offenbar notwendig, auch die Rolle der Bürokraten, hier verstanden als alle Mitarbeiter der öffentlichen Verwaltung, seien sie nun Ministerialbeamte, Lehrer, Politesse, Inspektorin im Sozialamt oder Krankenschwester, genauer zu differenzieren.

\subsubsection{Bürokraten}

Im Bild der autonomen Verwaltung kennen und verwirklichen nur ihre Mitarbeiter das Gemeinwohl und sind in der Lage, es unverfälscht umzusetzen. Aufgrund ihrer ethischen und professionellen Einstellung und Ausbildung sind sie in der Lage, öffentliche Ziele, Werte und Identität zu vermitteln und zu verkörpern. Das Bild der öffentlichen Verwaltung ist das einer Funktionselite oder eines Corps, charakterisiert durch normative Auswahl und Selbstselektion, gemeinsame Sozialisierung und die Beurteilung durch Vorgesetzte und "peer groups" (vgl. auch Mintzberg 1996). Wenn es ein klassisches Vorbild für diese Art der Organisation gibt, dann ist es die Kirche, die weniger durch strikte Regeln (obwohl es die natürlich auch gibt) und mehr durch gemeinsame Werte zusammengehalten wird. Das klassische moderne Beispiel sind die Gesundheitsberufe, aber auch Sozialarbeiter oder Denkmalschützer fallen in diese Kategorie. In abgeschwächter Form sind dies die von Frido Wagener beschriebenen Fachbruderschaften.

Für die hierarchische Verwaltung ermöglicht die auch dort vorhandene professionelle Einstellung und Ausbildung den Mitarbeitern, als neutrale und loyale Instrumente der demokratischen, rationalen Herrschaftsform zu handeln, berechenbar, stetig, effektiv usw., wie von Max Weber gefordert. Das Bild des öffentlichen Dienstes ist das einer präzise überwachten Maschine, in der Aufgaben zunächst isoliert, dann zugewiesen und schließlich gewissenhaft kontrolliert werden. Das klassische Vorbild ist hier das Militär oder auch die moderne, tayloristische Fabrik.

In der kooperativen Verwaltung ist die Verwaltung Partner und Widersacher der Politik sowohl bei Politikformulierung und -durchsetzung und insbesondere 
natürlich Partner und Widersacher organisierter Interessen. Politiker und Bürokraten bestimmen gemeinsam, was zu tun ist und wie es getan werden sollte. Das normative Bild ist das des Netzwerks. Es kommt darauf an, zu kommunizieren, Verbindungen zu schaffen und zu kooperieren. Gesteuert wird der loyale öffentliche Dienst in erster Linie durch Information und Verhandlung, aber auch durch die Personalauswahl und die Organisation als Verstetigung (oder Erschwerung) von Kommunikationsbeziehungen. Hier gilt zum einen das vormoderne Vorbild des Clans, aber vor allem das des schon beinahe post-modernen Netzwerkes.

Der öffentliche Dienst im Rahmen der responsiven Verwaltung ist schließlich bestimmt durch Kundenorientierung, Effizienz und Effektivität. Der moderne Bürokrat ist der öffentliche Manager und Entrepreneur, gesteuert durch Wettbewerb, Dialog und direkten Austausch mit der Gesellschaft und nicht zuletzt durch Geld. Das normative Bild ist das des Marktes, d. h. es kommt darauf an, öffentliche Bedienstete und administrative Einheiten so selbstständig wie möglich zu machen, sie mit Hilfe von Verträgen und - i.d. R. monetären - Anreizen dem Wettbewerb auszusetzen und für Erfolge direkt zu belohnen.

\subsubsection{Verwaltung und Demokratie}

Stellt sich schließlich die Frage, wie die demokratische Regierungsform mit diesen unterschiedlichen Steuerungsformen verbunden ist. Die autonome Verwaltung braucht bekanntlich keine Demokratie, sondern hält sie im Prinzip sogar für schädlich. Die Bindung an Politik wie Gesellschaft ist schwach, die ideale Regierungsform ist eine mit nicht-vorhandenen oder schwachen Politikern und starken, professionellen Angehörigen des öffentlichen Sektors. So stellen sich Chefärzte Krankenhäuser und Professoren Universitäten vor.

Die ideale Regierungsform der hierarchischen Verwaltung sind repräsentative Demokratie und Rechtsstaat, Willensbildung erfolgt Top-Down oder mit anderen Worten in der Form der „overhead democracy“. In dieser Regierungsform ist die Bindung an die Politik (Parlamente, Regierungen) stark, an die Gesellschaft (Interessengruppen, Einzelinteressen) eher schwach. Eine ideale Regierungsform mit starken Politikern und schwachen oder besser neutralen und loyalen Beamten, die keine Eigen- oder Partialinteressen vertreten.

Die kooperative Verwaltung ist die Regierungsform der organisierten Demokratie (Olsen 1983) und des liberalen Korporatismus. Sie ist gekennzeichnet durch starke Bindungen an die Politik und gleichzeitig an organisierte Interessen, sozusagen eine horizontale Demokratie. Idealerweise gibt es hier starke Politiker und starke Bürokraten, die sich in ihren normativen und faktischen Kompetenzen ergänzen.

Die ideale Regierungsform der responsiven Verwaltung könnte schließlich die partizipative, direkte Demokratie sein, gekennzeichnet durch Selbstorganisation der 
Gesellschaft und enge Verbindungen zwischen Bürokratie und Bürgern. Bindungen an die organisierte Politik sind schwach, dafür die an die Bürger umso stärker. Die responsive Verwaltung reagiert auf die „realen“ Bedürfnisse der Bürger, nicht auf die „bornierten“ Präferenzen der Politik. Im idealen Modell ist dies eine Regierungsform sowohl schwacher Politiker wie schwacher Beamter: Da die Verwaltung eng an die Wünsche und Bedürfnisse der Bürger gekoppelt ist, sind die Handlungs- und Entscheidungsspielräume beider Akteursgruppen äußerst beschränkt. Die Kunden entscheiden, was die Verwaltung unternimmt, nicht Politiker oder Bürokraten.

\subsubsection{Beispiele in Deutschland}

Nach dieser geballten Ladung normativer Idealtypen muss natürlich gefragt werden, ob und in welchen Formen diese Typen zumindest annäherungsweise in der Realität zu finden sind. Tatsächlich handelt es sich offenkundig nicht nur um eine historische Abfolge normativer Bilder der modernen, öffentlichen Verwaltung, sondern um unterschiedliche, widersprüchliche Vorstellungen, die aktuell unser Bild der Verwaltung bestimmen - und daher ein wichtiger Grund für die kontroversen normativen wie analytischen Einschätzungen der Verwaltung sind. Als Beispiel sollen dabei u.a. jeweils unterschiedliche Auffassungen einer modernen Universität herangezogen werden.

Das klassische Bild der autonomen Verwaltung ist offenkundig der überkommene Obrigkeitsstaat, aber es gibt diese Form auch in anderen Orientierungen. Es wurde bereits angedeutet, dass Strukturen der autonomen Verwaltung im demokratischen System der Bundesrepublik Deutschland durchaus wohlauf und erkennbar sind. Gemeint ist damit das, was gelegentlich als der professionell-bürokratische Komplex bezeichnet wird, also die Wahrnehmung öffentlicher Aufgaben durch mehr oder weniger etablierte Professionen oder Fachbruderschaften. Ärzte, Sozialarbeiter, Kulturschaffende, Denkmal- oder Umweltschützer sind nicht selten davon überzeugt, dass ihre Aufgabenwahrnehmung den „richtigen“, professionellen (ethischen, wissenschaftlichen) Standards entspricht und entsprechen sollte, und dass diese Standards insbesondere durch unqualifizierte, egoistische, kurzsichtige und unvorhersehbare politische Eingriffe gefährdet sind. Ziel dieser Professionen ist daher oft keineswegs eine Stärkung demokratischer Mitbestimmung, sei es über die organisierte Politik, sei es über direkte Demokratie, sondern eher, die professionelle Aufgabenwahrnehmung vor politischer Einflussnahme und letztendlich auch vor Transparenz zu beschützen. Autonomie ist dabei keineswegs gleichbedeutend mit Unabhängigkeit (das wäre Autarkie), „Autonom ist vielmehr, wer die in sozialen Beziehungen immer vorhandene Abhängigkeit zum eigenen Vorteil nutzen kann" (Czada 1992, S. 183). Öffentliche Theater, Krankenhäuser aber auch Universitäten und Sozialämter verwenden einen nicht unerheblichen Teil ihrer Energie darauf, 
ihre (sicherlich keineswegs unbegrenzte) Autonomie gegenüber „willkürlichen“ politischen und gesellschaftlichen Eingriffen zu verteidigen. Auch und insbesondere die Politik ist eine problematische Umwelt dieser vom Anspruch her „autonomen“, professionell gesteuerten Bereiche.

Ein typisches Beispiel für diese Art öffentlicher Organisation ist auch die Universität in ihrem Bild als „Gelehrtenrepublik “, die sich jegliche politische Einflussnahme verbittet, und insgesamt alle öffentlichen wissenschaftlichen Einrichtungen, die stolz darauf sind, ihre Aufgaben ohne politische Einflussnahme wahrzunehmen. Aber zu denken ist zum Beispiel auch an Fachbehörden wie Umweltämter, die sich mit aller Macht dagegen wehren, in eine allgemeine Verwaltung (z. B. des Kreises) eingeordnet $\mathrm{zu}$ werden. In unserem System ist die autonome Verwaltung nie wirklich autark, aber die Erhaltung und Erweiterung ihrer Autonomie ist eines ihrer wichtigsten Ziele. Typische Reformvorschläge aus dieser Verwaltung zielen daher vorrangig auf die Stärkung der professionellen Autonomie, z. B. durch bessere, professionell kontrollierte Ausbildung, professionalisierte Zugangsbeschränkungen, aber auch z.B. durch dezentrale Ressourcenverantwortung und Befreiung von allgemeinen politisch-administrativen Vorgaben. Die modernste Form der autonomen Verwaltung sind unabhängige Regulierungsbehörden, die ihre Aufgaben ohne „unsachliche“ politische Interventionen wahrnehmen können.

Die hierarchische Verwaltung ist das Idealbild des demokratischen Verfassungsstaats, und selbstverständlich ist sie auch empirisch relevant. Ihren reinsten Ausdruck findet sie in der klassischen Ministerverantwortlichkeit, aber auch der kommunalen Selbstverwaltung. Es gibt ohne Zweifel viele Felder, die durch legislative Programmierung verbunden mit klassischer Rechts- und Fachaufsicht gekennzeichnet sind, z. B. Polizei, Rentenversicherung, Bauaufsicht oder der gesamte Schulbereich. Aber genau diese Bereiche sind auch durch eigene professionelle Standards und nicht zuletzt durch Implementationsprobleme und „Vollzugsdefizite“ gekennzeichnet. Recht ist, insbesondere in unserer Verwaltungskultur, immer noch das zentrale Steuerungsmedium innerhalb des politisch-administrativen Systems, und starke, d.h. mit erheblichen Handlungsspielräumen ausgestattete hierarchische Steuerung verbunden mit schwacher, d. h. in ihren Handlungsspielräumen beschränkte Bürokratie ist keineswegs ausgestorben, aber dennoch gibt es auch und gerade in streng durchnormierten Bereichen, wie z.B. der Steuerverwaltung, Tendenzen der Autonomisierung, der verhandelnden Verwaltung und schließlich auch der responsiven Aufgabenerfüllung („angemessener Gesetzesvollzug“).

Wenn wir wiederum die Universitäten als Beispiel nehmen, ist dies das Bild der Universität als „nachgeordneter Behörde“, von der demokratisch legitimierten Politik über Rechts- und Fachaufsicht und insbesondere über das jährliche Budget und den Stellenplan und deren Kontrolle stark beeinflusst, wenn nicht bis zur 
Dysfunktionalität in ihren Aufgaben behindert. Das Umweltamt auf lokaler oder regionaler Ebene ist selbstverständlich durch umfangreiche Rechtssetzung und das Budget gesteuert, aber auch durch die hierarchische Einordnung in eine Behörde. Reformvorschläge für diese Form der Verwaltung richten sich insbesondere an die Stärkung der politischen Steuerung, z. B. durch die Verbesserung der politischen Führung (Planung, Informations- und Konfliktregelungskapazität, „aktive Politik“), an eine verbesserte Rechtssetzung (Rechtsbereinigung, Entbürokratisierung) oder auch an Verbesserungen der Aufbau- (schlankere, straffere Hierarchie, Optimierung der Leitungsspanne, Großreferate etc.) und Ablauforganisation (Verminderung von Schnittstellen, Beschleunigung von Verfahren). Auch die Reduzierung von Politikverflechtung und Mischfinanzierung bis hin zu Spitzenpositionen auf Zeit gehören in diesen Reformkanon.

Die kooperative Verwaltung ist offenkundig das Lieblingskind der empirischen Politik- und Verwaltungsforschung. Ihr Prototyp ist die moderne Ministerialverwaltung, aber sie findet sich auch im Bereich der Kommunen und nicht zuletzt im weiten Feld der Quagos und Quangos (Hood/Schuppert 1988). Klassische Beispiele sind die Umweltpolitik, die Arbeitsmarkt- und Gesundheitspolitik, wo inzwischen vielfach nachgewiesen wurde, dass öffentliche Verwaltung nicht nur eine entscheidende Rolle bei der Formulierung von Politikinhalten spielt, sondern zum einen ein wichtiger Akteur ist bei der Konstruktion und ständigen Rekonstruktion der Politiknetzwerke und Verhandlungssysteme, zum anderen auch im Rahmen der Politikdurchführung viel mehr mit Adressaten verhandelt und koordiniert als Gesetze einfach anwendet und anweist. Insbesondere die Implementationsforschung hat gezeigt, dass öffentliche Verwaltungsorganisationen durch legislatorische Programme nur sehr ungenügend gesteuert werden können, und dass erhebliche und erweiterte Handlungsspielräume im Rahmen der Implementation von Programmen keineswegs nur negativ zu bewerten sind. Im Gegenteil, eine sklavische Implementation der vorliegenden Programme deutet weniger auf starke politische Determination und Steuerung, sondern eher auf Unfähigkeit oder Unwilligkeit im Rahmen des Implementationsprozesses zu lernen und notwendige Anpassungsleistungen zu erbringen.

Am Beispiel der Universität wäre dieses Steuerungsmodell die moderne durch einen externen Hochschulrat gesteuerte und so von kurzfristiger politischer Einflussnahme und Eigeninteressen weitgehend abgekoppelte Hochschule, die eng mit ihrer Umgebung (z. B. Stadt, regionale Wirtschaft, Verbände) verflochten ist. Diese ideale Hochschule erhält die Prämissen ihres Handelns nicht allein aus dem Ministerium, sondern ebenso oder stärker durch die gesellschaftlichen Kräfte ihrer Umwelt. Ähnlich zeichnet sich das kooperative Umweltamt zum einen durch enge Verbindungen mit der Politik aus, zum anderen durch intensive gesellschaftliche 
Kontakte. Umweltschutz wird im Dialog und als Verhandlungsprozess betrieben, gleichzeitig werden intensive Kontakte mit der eigenen Klientel und insbesondere mit den relevanten Interessengruppen betrieben. Sollte die gesellschaftliche Organisation des Umweltschutzes noch schwach sein, leistet die Verwaltung Hilfe bei der Organisation dieser gesellschaftlichen Interessen (Müller 1986, Czada 1991). Typische Reformvorschläge zur Stärkung und Verbesserung der kooperativen Verwaltung bewegen sich in Richtung verstetigter Konsultations- und Kooperationsgremien, größerer und erleichterter Mobilität zwischen öffentlichem und privatem Sektor, mehr Projektorganisation bis hin zu Globalhaushalten.

Die responsive Verwaltung, gesteuert durch Kunden und öffentliche Entrepreneure ist schließlich in weiten Teilen das Idealbild der Diskussionen über neue Steuerungsmodelle. Aber auch bisher gibt es bereits diese Art der Verwaltung, die sich durch ihre Bürgernähe definiert, zumindest einen Teil ihrer Ressourcen durch Gebühren oder andere Einnahmen selbst deckt und ihren Erfolg vorrangig an ihrer Akzeptanz bei ihren Klienten, weniger der Politik misst. Beispiele sind öffentliche Kindergärten, Altersheime, Pflegedienste, externe wie interne Dienstleister (Reinigung, Gebäudemanagement, Fuhrpark etc.), aber auch Katasterämter, Statistische Landesämter und selbst Polizeidienststellen können sich als responsive Verwaltung „neu erfinden“. Die Politik wird in diesem Modell geschwächt, sobald Ressourcen eigenständig eingenommen und eingesetzt werden können, die Bürokratie ist schwach, sobald sie sich in ihren Leistungen und Angeboten an den Wünschen der Klienten orientieren muss und insbesondere sobald Klienten die Möglichkeit haben, zwischen vergleichbaren öffentlichen und privaten Angeboten zu wählen. Demgegenüber ist die Bindung an die Gesellschaft stark, nicht nur über Preise und Nachfrage, sondern z. B. auch durch Beiräte, Bürgerbefragungen oder öffentliche Leistungsvergleiche.

Die Universität als responsive Organisation wäre die moderne Service-Universität, die einen nicht unbeträchtlichen Teil ihrer Einnahmen durch Studiengebühren und Forschungsförderung selbst erwirtschaften muss, die sich gleichzeitig ihre Studenten selbst aussuchen kann und durch starke Präsidenten und Dekane geleitet wird. Das Umweltamt dieser Art finanziert einen nicht unerheblichen Teil seines Haushalts durch Dienstleistungen, außerdem misst es regelmäßig sowohl die objektive Umweltqualität wie die subjektive Zufriedenheit der Bürger mit seinen Leistungen und vergleicht sich mit anderen Umweltämtern. Was die typischen Reformvorschläge in diese Richtung angeht, braucht hier das umfangreiche Arsenal des Neuen Steuerungsmodells nicht wiederholt zu werden, von der dezentralen Ressourcenverantwortung über Kosten-Leistungsrechnung, Produktorientierung, Qualitätsmanagement und Leistungsvergleichen bis hin zu Ausschreibungen, Markttests und Outsourcing (siehe ausführlich 5.2.4). 
Die Diskussion dieser „normativen Bilder“ der Verwaltung, und insbesondere der darin enthaltenen unterschiedlichen Vorstellungen der Verbindungen und Funktionen von Politik, Verwaltung und Gesellschaft, zeigt, dass es keine eindeutigen und invarianten Funktionszuweisungen gibt, und auch nicht geben kann. Das Bild der öffentlichen Verwaltung verändert sich mit dem Bild des modernen, demokratischen Verfassungsstaates. Wenn sich, z. B. im Gefolge der Diskussionen über Management und Governance (s. o. 2.5), neue und veränderte Vorstellungen staatlicher Steuerung und der Bedeutung staatlicher und gesellschaftlicher Institutionen entwickeln, hat dies selbstverständlich auch erhebliche Auswirkungen auf unser Verständnis der Verwaltung. Gleichzeitig wird aber auch deutlich, dass es "schon immer" und auch in Zukunft sehr unterschiedliche Vorstellungen von den Aufgaben, der Autonomie und der gesellschaftlichen Verankerung öffentlicher Organisationen gibt und geben wird. Gerade dies macht den Reiz und die offenkundige Relevanz der modernen Verwaltungswissenschaft aus, die daher immer sowohl eine analytische wie auch normative Wissenschaft sein muss.

\subsection{Politikberatung}

Politikberatung ist ein schillernder Begriff, Politikberater ist keine geschützte Berufsbezeichnung (siehe als Überblick Falk et.al. 2019). Nach Göttrik Wewer (2014), der sowohl Erfahrungen als Wissenschaftler, Top-Beamter und Lobbyist hat, gibt es kaum eine Berufsgruppe, die ständig so viele gute Ratschläge bekommt wie Politiker: von Parteifreunden, von Journalisten, von Lobbyisten, aus dem Parlament, der Wirtschaft, der Wissenschaft, der Familie, bei Empfängen, auf der Straße, im Taxi, und natürlich auch in Form von Expertisen, Memoranden, Gutachten oder auch Anhörungen und Beiräten.

\subsubsection{Ist denn alles Politikberatung?}

Sven Siefken (2010) hat daher darauf hingewiesen, dass Politikberatung als „Modethema der Politikwissenschaft" zu einer inflationären Verwendung des Begriffs und zur begrifflichen Unschärfe geführt hat, „Es gilt offenbar: Politikberatung ist Beratung von allen, durch alle und zu allem!“ (S. 129). Der expandierende Begriff der Politikberatung bedarf, wie alle wichtigen Begriffe, einer realistischen Beschreibung und klaren Eingrenzung des Gegenstandes. 
Für die Verwaltungswissenschaft ist Politikberatung aus zwei miteinander verbundenen Gründen von besonderer Bedeutung. Zum einen ist offenkundig, dass professionelle Bürokratien die wichtigsten Berater der Politik und unserer Politiker sind, denn es ist ihre zentrale Aufgabe, die in unseren extrem arbeitsteiligen und spezialisierten öffentlichen Organisationen vorhandenen umfangreichen Informationen zu filtern, zu verdichten und entscheidungsreif zu präsentieren. Zum Teil produziert der öffentliche Sektor in einer Vielzahl von Forschungsanstalten, Stiftungen etc. auch eigene wissenschaftliche Politikberatung (sog. Ressortforschung). Zum anderen ist ein Großteil der normalerweise unter der Überschrift Politikberatung laufenden Aktivitäten in Wirklichkeit Verwaltungsberatung. Die Ergebnisse von Gutachten, Expertenkommissionen, Anhörungen, Enqueten usw. sind in aller Regel so umfangreich und so speziell, dass sie wiederum nur von den Experten in den Verwaltungen im Detail gelesen und verarbeitet werden können, die diese Ergebnisse dann wiederum für die politischen Spitzen aufbereiten. „Der Großteil der Tätigkeit der wissenschaftlichen Berater erfolgt ohne die Aufmerksamkeit der Medien, geräusch- und ereignislos zumeist auf der Referentenebene der Ministerien und nachgeordneten Behörden“" (Weingart 2019, S. 68). Das ist auch gar nicht zu beklagen, denn das ist der Sinn arbeitsteiliger Organisationen.

Politik- und Verwaltungsberatung kann nach einer Reihe unterschiedlicher Kriterien unterschieden werden, z.B.

- wissenschaftlich oder interessengeleitet,

- dauerhaft oder zeitlich begrenzt,

- kommerziell oder gemeinnützig,

- von Individuen oder spezialisierten Organisationen,

- bestellt oder ungefragt usw.

Allerdings zeigt schon eine oberflächliche Betrachtung, dass diese Unterscheidungen alles andere als einfache Dichotomien sind, sondern alle auf einem Kontinuum liegen. Welche wissenschaftliche Untersuchung ist vollkommen interessenfrei, und wie sieht es mit wissenschaftlichen Expertisen des Instituts der Deutschen Wirtschaft oder des Öko-Instituts in Freiburg aus? Ist ein Gutachten, weil es von den Gewerkschaften oder dem BUND bestellt und bezahlt wurde, unwissenschaftlich? Sind bei universitären An-Instituten bestellte Gutachten kommerziell oder gemeinnützig, und sagt das etwas über ihre Qualität aus? Idealtypisch lassen sich aufgrund der jeweils vorherrschenden Struktur der Nachfrage drei große Felder von Politikberatung unterscheiden (vgl. Wewer 2014; Siefken 2007, S. 38ff.): 
- Organisatorische und institutionelle Beratung, die sich zum einen auf die Verbesserung von Abläufen und Strukturen, der Effizienz und Effektivität von Personal oder Finanzen öffentlicher Organisationen bezieht, z. B. von Verwaltungen, Universitäten, öffentlichen Unternehmen etc. Dies ist eine Domäne der Betriebswirtschaft sowie kommerzieller Managementberater und Beratungsfirmen (man könnte auch von Management-Beratung sprechen). Zum anderen ist dies aber auch Beratung zum institutionellen Aufbau des politisch-administrativen Systems (etwa des deutschen Föderalismus), zu Fragen der Abgrenzung zwischen öffentlichem und privatem Sektor (z. B. Privatisierung) oder etwa zu allgemeinen Verfahren der besseren Regulierung oder Gesetzesfolgenabschätzung. Im Sinne der klassischen Politik-Unterscheidung könnte man hier von Polity-Beratung sprechen (d.h. es geht um „Verwaltungspolitik“ im Sinne von Policies zur Veränderung der Polity).

- Strategische und taktische Beratung, bei der es um die Chancenerhöhung im politischen Wettbewerb (etwa im Wahlkampf) geht, also die klassische Domäne von Befragungsfirmen (wie Infas, Forschungsgruppe Wahlen) oder auch von Public-Relations-Beratern (Politics-Beratung).

- Materielle oder Programmberatung beschäftigt sich schließlich mit der ex ante Ausgestaltung und Planung oder auch ex post Evaluierung und Bewertung politischer Programme und Vorhaben, also von Politikinhalten in allen möglichen Politikbereichen, von der Umwelt- über die Arbeitsmarkt- bis zur Kultur- und Klimapolitik. Dies ist eine Domäne sowohl der natur- wie der sozialwissenschaftlichen oder ökonomischen Politikfeld-Forschung, der jeweils „zuständigen“ Disziplinen, aber natürlich auch der jeweils betroffenen Interessen und Akteure (Policy-Beratung). Dazu gehört auch die klassische Fachberatung, etwa zur Klärung technischer Fragen im Bau- oder Umweltbereich, z. B. welche Kosten eine Bauleistung verursachen wird, wie bestimmte Ökosysteme geschützt werden können, Gutachten zu Sicherheitsfragen oder Bebauungsplänen oder auch die juristische Beratung bei Vertrags- und Schadensersatzfragen (technische Beratung oder Expertise).

Offensichtlich gibt es Überschneidungen zwischen diesen Kategorien - auch materielle Politikberatung kann der Verbesserung von Wahlchancen dienen, oder strategische Beratung der Evaluierung bestimmter Maßnahmen, veränderte Policies betreffen i.d. R. auch institutionelle Fragen usw. -, aber die Unterscheidungen verdeutlichen doch den Umfang und die unterschiedlichen Funktionen von Politikberatung.

Siefken weist allerdings darauf hin, dass diese schon sehr weite Definition von Politikberatung in den letzten Jahren noch erweitert worden ist, indem darunter auch 
die Beratung von Unternehmen oder Interessengruppen zur besseren Vertretung ihrer Interessen und Standpunkte gefasst wird. Diese Ausweitung des Begriffs wurde

„nicht zuletzt von interessierter Seite, namentlich den expandierenden Lobbyingund Public-Relations-Agenturen, betrieben, die mit dem neutraleren Begriff der Politikberatung offenbar ein besseres Image für ihre Dienstleistungen erhoffen, als es der im Ruf einseitiger Interessenspolitik stehende Begriff des Lobbying ermöglicht. Sehr aktiv war in dieser Erweiterung des Beratungsbegriffes insbesondere die ,Deutsche Gesellschaft für Politikberatung (degepol), die Marketingfunktionen für ihre rund hundert Mitglieder aus dem Lobbying- und Agentur-Bereich übernimmt, in der wissenschaftlichen und publizistischen Außendarstellung aber nicht selten - völlig unzutreffend - als ,Sprachrohr der Politikberater" wahrgenommen wird" (Siefken 2010, S. 131).

Er schlägt daher vor, den Begriff der Politikberatung analytisch zu begrenzen, Politikberatung also sinnvollerweise nicht als Beratung aller möglichen Adressaten zu politischen Themen zu verwenden, sondern darunter nur die Beratung politischer Entscheidungsträger zu verstehen, also derjenigen, die verbindliche allgemeingültige Entscheidungen treffen können. In diesem Sinne soll der Begriff auch hier verwendet werden.

\subsubsection{Akteure der Politikberatung}

Im Folgenden soll es um diese offizielle und organisierte Politikberatung gehen, die also von Verbänden, Unternehmen, Kommissionen, Wissenschaftlern, professionellen Beratern und ähnlichen zur Verfügung gestellt wird. Eine einfache Unterscheidung fragt nach den zentralen Anbietern von Politikberatung. Hier kann man folgende grobe Kategorien unterscheiden (vgl. Wewer 2014; Siefken 2007; als Überblick auch Falk et.al. 2019, S. 14):

- Kommerzielle Berater, die von der Beratung „leben müssen“, die also auf eine relevante Nachfrage reagieren. Hierzu gehören Consulting-Firmen (wie etwa Roland Berger und McKinsey), aber auch Stadtplaner, Ingenieure oder EDV-Firmen, private Institute (wie Prognos oder Battelle) und die professionellen Meinungs- und Umfrageforscher (in der Begrifflichkeit der Statistik sind dies „Vollerwerbsbetriebe“).

- Potenzielle Anbieter sind etwa Wissenschaftler und wissenschaftliche Institute an Universitäten, Max-Planck- oder Fraunhofer-Institute, die zwar nicht von Politikberatung leben, aber diese Aufträge doch gut als Drittmittelforschung und zur Erhöhung der eigenen Reputation gebrauchen können („Zuerwerbsbetriebe“). 
- Spezialisierte Beratungsinstitutionen sind im Prinzip dazu geschaffen, Politikberatung in einem bestimmten Feld anzubieten, aber reagieren nicht nur auf eine bestimmte Nachfrage; dazu gehören Einrichtungen der Ressortforschung (Umweltbundesamt, Bundesforschungsanstalten, öffentliche Labore etc.), aber auch die Stiftung Wissenschaft und Politik in der Außenpolitik, das Institut für Arbeitsmarkt- und Berufsforschung oder die wirtschaftswissenschaftlichen Forschungsinstitute, international etwa die OECD („Nebenerwerbsbetriebe“ mit einer staatlichen Grundfinanzierung).

- Eigenständige Denkfabriken, wie etwa Brookings in den USA oder das Policy Institute in Großbritannien (vgl. Gellner 1995, Braml 2004) sind in Deutschland noch rar, aber dazu könnte man etwa die Bertelsmann Stiftung zählen. Eigenständig bedeutet durchaus nicht immer unparteiisch (auch nicht bei den ausländischen Beispielen), hierzu zählen also auch Institute von Verbänden und Interessengruppen, die vorrangig deren Positionen untermauern (Karl-Bräuer-Institut des Bundes der Steuerzahler, Institut für Mittelstandsforschung) die Forschungsinstitute der parteinahen Stiftungen oder durch eine bestimmte inhaltliche „Richtung" festgelegte Institute (etwa das Freiburger Öko-Institut).

Das Angebot ist also vielfältig und bunt, auch wenn die Zahl derjenigen, die sich ausschließlich oder zu großen Teilen durch Politikberatung finanzieren, noch recht klein sein dürfte. Schließlich ist noch zu unterscheiden, wie diese Politikberatung präsentiert wird. Neben den klassischen Formen der Gutachten, Expertisen, Memoranden etc. geht es hier insbesondere um Beiräte, Kommissionen u.ä. Auch hier ist die Anzahl und Vielfalt sehr unübersichtlich, und war insbesondere durch verschiedene bekannte und kontroverse Beratungsgremien - wie etwa die Süssmuth-Kommission zur Zuwanderung, die Hartz-Kommission zur Arbeitsmarktpolitik, die Rürup-Kommission zur Renten- und Gesundheitspolitik - zunehmender Kritik ausgesetzt. Aber auch im Koalitionsvertrag der Großen Koalition vom Februar 2018 werden insgesamt 16 neue Kommissionen angekündigt, von der bekannten „Kohlekommission“ bis hin zu einer Expertenkommission zum Thema „Antiziganismus“.

In der bisher umfassendsten und besten Untersuchung zu Expertenkommissionen kann Sven Siefken $(2007,2019)$ zeigen, dass die Anzahl solcher Beratungsgremien im Gegensatz zur populären Wahrnehmung vermutlich langfristig kaum gestiegen ist. Bei aller Unsicherheit der Datenlage liegt die Zahl offizieller Beratungsgremien des Bundes seit den neunziger Jahren etwa bei 150, während in den siebziger Jahren bis zu 350 gezählt wurden. Was sich allerdings erheblich verändert hat, ist die öffentliche Wahrnehmung dieser Gremien. Während in den neunziger Jahren jährlich etwa 200 
bis 240 Berichte über solche Gremien in den deutschen "Qualitäts-Tageszeitungen“ erschienen, sind es seit dem Jahr 2000 zwischen 500 und über 700.

Siefken macht auch einen hilfreichen Vorschlag zur Vereinheitlichung der sehr uneinheitlichen Begrifflichkeit.

\begin{tabular}{lll}
\hline Besetzung $^{76}$ & dauerhaft & zeitlich begrenzt \\
\hline Wissenschaft, Interessengruppen & Beirat & Expertenkommission \\
\hline Regierung, Verwaltung & Arbeitskreis & Projektgruppe \\
\hline Parlament & Ausschuss & $\begin{array}{l}\text { Enquete-Kommission } \\
\text { Untersuchungsausschuss }\end{array}$ \\
\hline
\end{tabular}

Abb. 56 Unterschiedliche Expertengremien

Quelle: nach Siefken 2003, S. 496

Beispiele für Beiräte wären so z.B. der Wissenschaftliche Beirat im Finanzministerium, die Monopolkommission, der Normenkontrollrat oder der Nationale Ethikrat, während ein typischer Arbeitskreis der Arbeitskreis Steuerschätzung wäre und das Parlament einen Großteil seiner Arbeit bekanntlich in festen Ausschüssen organisiert. Beispiele für zeitlich begrenzte Gremien wären die berühmt-berüchtigten Hartz- oder Rürup-Kommissionen, während Beispiele für eine Projektgruppe etwa die Projektgruppe Regierungs- und Verwaltungsreform, der Staatssekretärsausschuss für Bürokratieabbau oder die Kommission zur Gemeindefinanzreform wären. Enquete-Kommissionen vereinen bekanntlich externe Experten und Parlamentarier, wobei die Zusammensetzung zwischen Bundestag und Landtagen etwas differiert. Expertengremien sind weder ein spezifisch deutsches noch ein neues Phänomen, sondern gehören zum festen „Inventar“ der Politikberatung, sie erfüllen alle möglichen Funktionen, von der Klärung wissenschaftlicher Grundlagen über die Externalisierung von Verhandlungen bis hin zur Symbolpolitik, von der Problemdefinition bis zur Legitimation (Siefken 2019, S. 158f.; Wewer 2014, S. 486). Sie sind allerdings, gerade weil es sich dabei in aller Regel nicht um rein wissenschaftlich besetzte Gremien handelt, sondern in aller Regel „Fachleute“ und Vertreter gesellschaftlicher Gruppen in ihnen vertreten sind, ein wichtiges Element der schwierigen Beziehung zwischen Wissenschaft und Politik.

76 Jeweils die Mehrheit der Mitglieder sollte der jeweiligen Gruppe entstammen. 


\subsubsection{Wissenschaft und Politik}

Die moderne Gesellschaft wird oft als Wissensgesellschaft bezeichnet, und gerade die Corona-Krise hat gezeigt, dass politische Entscheidungen in vielen, wenn nicht allen Bereichen ohne wissenschaftliche Beratung nicht mehr möglich sind. Die Frage, wie das Verhältnis zwischen Wissenschaft und Politik empirisch ausgestaltet ist und wie es normativ ausgestaltet werden sollte, ist daher von eminenter Bedeutung. Die theoretische Diskussion um die Beziehungen zwischen politisch-administrativer Praxis und Politikberatung wird seit den sechziger Jahren durch drei ursprünglich von Jürgen Habermas unterschiedene Beratungsmodelle bestimmt: dem dezisionistischen, dem technokratischen und dem pragmatistischen Modell der Politikberatung (Habermas 1963; zusammenfassend Siefken 2007, Blätte 2019, S.29):

- Dem technokratischen Modell zufolge wird der politische Entscheidungsspielraum aufgrund zunehmender „Sachzwänge“ immer mehr auf den von der Wissenschaft vorgezeichneten „one best way“ reduziert. Letztendlich entscheiden Experten und die Wissenschaft alle wichtigen Fragen, es gilt eine Expertokratie, Politik legitimiert nur noch.

- Dagegen verbleibt die Entscheidung im dezisionistischen Modell vollständig in der Politik, die Ziele und Wege der Beratungstätigkeit bestimmt und der Wissenschaft als „hired guns“ die bloße Dienstleistung überlässt. Die Wissenschaft ist Handlanger der Politik und wird von dieser für ihre Zwecke genutzt.

- Im pragmatistischen (nicht ,pragmatischen“) Modell wird ein kritisches Wechselverhältnis angestrebt: Praktiker bzw. Politiker und Wissenschaftler vollziehen im Forschungs- und Entscheidungsprozess immer neue, nicht nur vom Sachzusammenhang, sondern auch von Wertungen begleitete Wahlakte. Entscheidungen entstammen der Kooperation und des gegenseitigen Lernens.

Als am ehesten realistisch und auch normativ erwünscht gilt seit langem das pragmatistische Modell. Auch wenn die Interessen der beiden Pole höchst unterschiedlich sein mögen - Wissenschaftlern mag es um „Wahrheit“, die Anwendung und Weiterentwicklung von Methoden und Reputation gehen, Praktiker erhoffen sich Argumentations- und Rechtfertigungshilfe -, ist die Chance eines sachdienlichen Austausches hier tendenziell gegeben, werden neue Forschungen angeregt und scheinbar gesichertes Handeln problematisiert. Insgesamt ist allerdings umstritten, ob und wie wissenschaftliche, insbesondere sozial- und politikwissenschaftliche Analysen und Theorien überhaupt von der politisch-administrativen Praxis genutzt werden können. Die Probleme der Bereitstellung und Nutzung von Wissen 
werden dabei unterschiedlich verortet. Unterscheiden kann man in der aktuellen Diskussion drei sozialwissenschaftliche Ansätze (vgl. zum Folgenden Blätte 2019). Akteurzentrierte Ansätze sehen Politikberatung als Austausch handlungsrelevanten Wissens zwischen Individuen und vor allem Organisationen. Hier gelten dann alle Restriktionen der oben bereits diskutierten begrenzten Rationalität (bounded rationality), der selektiven Perzeption usw. (vgl. oben 4.3). Politikberatung ist durch begrenztes Wissen und Informationsverarbeitungskapazitäten eingeschränkt, durch organisatorische Gegebenheiten, aber auch durch Defizite der gegenseitigen Kommunikation und Wahrnehmung und gegensätzliche Interessen. Betont werden in diesen Studien Unzulänglichkeiten

- der Angebotsseite, d.h. des Wissenschaftssystems, z. B. weil die von der Wissenschaft angebotenen Darstellungen und Erklärungen unzureichend empirisch und theoretisch fundiert, d. h. unbrauchbar sind, weil sie zu spät kommen oder weil Wissenschaft sich, aufgrund falscher Anreize, unzureichend mit praxisrelevanten Problemen beschäftigt;

- der Kommunikation zwischen Wissenschaft und Praxis, z. B. weil unterschiedliche Fachsprachen und Jargons benutzt werden und man sich daher nicht verständigen kann oder will; und

- der Nachfrageseite, d. h. des politisch-administrativen Systems, z. B. weil durch ausgeprägte juristische Orientierung der öffentlichen Verwaltung Ansprechpartner auf der Seite der Praxis fehlen, wissenschaftliche Ergebnisse nicht wahrgenommen oder bewusst ignoriert werden oder vorrangig zur Legitimation bereits gefasster Entscheidungen verwendet werden.

Systemtheoretische Ansätze gehen in ihrer reinen Form davon aus, dass soziale Systeme in ihrer eigenen Logik , autopoetisch “ gefangen sind, und es daher keinen sinnvollen Austausch zwischen Wissenschaft (Leitdifferenz Wahrheit/Unwahrheit) und Politik (Machterhalt/Machtverlust) geben kann (Luhmann 1983):

„Politische Akteure können nicht umhin, wissenschaftliche Erkenntnisse unter dem Gesichtspunkt des politischen Nutzens zu bewerten. Der strategische Einsatz des Wissens im Ringen um Macht dominiert. Wissenschaftliche Akteure, denen der Wahrheitsgehalt ihrer Erkenntnisse als Leitstern gilt, müssen darin einen sachfremden Missbrauch der Ergebnisse ihres Schaffens wittern“ (Blätte 2019, S.31).

Erfolgreiche Politikberatung ist daher, wenn nicht prinzipiell unmöglich, dann zumindest äußerst unwahrscheinlich.

Wissensbasierte Ansätze betonen schließlich die Bedeutung spezifischer Deutungsmuster, die sich gemeinsam zwischen Politik und Wissenschaft entwickeln. 
Schlagworte sind Diskurskoalitionen, Epistic Communities oder Advocacy Coalitions, Diskurse, Ideen und Institutionen stehen im Zentrum der Forschung (Nullmeier 2014). Die Beziehung zwischen Politik und Beratern wird als eine Konflikt- bzw. Machtkommunikation gesehen, deren Gegenstand Definitionsmacht im Hinblick auf die Legitimation politischer Entscheidungen ist:
„Die Bedeutung der wissenschaftlichen Politikberatung ergibt sich aus dem Um- stand, dass politische Macht, insbesondere in modernen Demokratien, auf einer doppelten Legitimation beruht: auf der Delegation der Macht durch Wahl und auf der Rationalität politischer Entscheidungen durch den Bezug auf gesichertes und in der Wissenschaft konsentiertes Wissen. (...) Entscheidungen, die offenkundig gesichertem Wissen zuwiderlaufen, haben wenig Aussicht auf öffentliche Akzeptanz" (Weingart 2019, S.71f.).

Gesichertes Wissen ist allerdings ein knappes Gut (vgl. zum Folgenden die Zusammenfassung von Weingart 2019, S.73ff.). In den Naturwissenschaften ist der Grad des wissenschaftlichen Konsenses in vielen Fällen vergleichsweise hoch, sodass es seltener zu grundlegenden Expertenkonflikten und dadurch zu Politisierung der Beratung kommt. Aber auch hier gibt es Unsicherheit des Wissens, Kontextgebundenheit und Nichtwissen. Die aktuellen Debatten über Klimafolgen oder die Bekämpfung des Coronavirus sind dafür gute Beispiele. In Politikbereichen, die in die Zuständigkeit der Sozialwissenschaften fallen, ist dies noch viel ausgeprägter:
„In diesen Bereichen ist das relevante Wissen zu einem weit geringeren Grad in den entsprechenden Gemeinschaften konsentiert. Die Wert- und Interessenbezüge der involvierten Entscheidungen (u. a. soziale Gerechtigkeit, Besitzstandswahrung) reichen weit in weltanschauliche Deutungssysteme hinein und sie lassen sich infolgedessen leichter politisieren“" (ebd., S.73).

Weingart spricht in diesem Zusammenhang von Expertise, also auf politische Probleme hin aufbereitetes und interpretiertes Wissen. Berater können sich nicht auf die Präsentation von Fakten und Theorien beschränken, sondern müssen deren Bewertung mitliefern,
„Beratungsprozesse sind aus dieser Perspektive Aushandlungsprozesse, in denen es zwischen Experten und Entscheidern um die Angemessenheit des Wissens im Hinblick auf Entscheidungsprobleme geht“ (ebd., S. 75).

Politikberater sind dabei weder reine Wissenschaftler noch Handlanger, sondern befinden sich auf einem Kontinuum zwischen vollkommener Autonomie auf der einen, und politischer Abhängigkeit auf der anderen Seite. Ihre Position auf diesem 
Kontinuum können sie, zumindest in Demokratien, weitgehend selbst bestimmen (auch wenn natürlich finanzielle Anreize und Reputation immer eine Rolle spielen).

Für die verwaltungswissenschaftliche Beratung gilt dies in besonderem Maße, denn Verwaltungswissenschaft hat sich seit Jahren vielfach mit Verwaltungsreformen beschäftigt, ist in vielen Bereichen vor allem also Verwaltungsreformwissenschaft. ${ }^{77}$ Die moderne Verwaltungswissenschaft und Policy-Forschung ist daher mit dem Schlagwort "praktische Fragen und theoretische Antworten" charakterisiert worden (Jann 2009, siehe hierzu auch die Überblicksartikel zu Public Administration and Practices von Pollitt/Ongaro/Saporito in Bouckaert/Jann 2020).

Wie verwaltungswissenschaftliche Beratungsprozesse im Geflecht zwischen Parteienwettbewerb, beschränkten Ressourcen, Implementationsproblemen durch vertikale und horizontale Verflechtungen sowie Eigeninteressen der Implementeure konkret ablaufen und welche Logik sich wann durchsetzt, ist bisher allerdings kaum erforscht. Ein gutes Beispiel ist die verwaltungswissenschaftliche Beratung der letzten Jahre im Bereich der Verwaltungspolitik der Bundesländer (vgl. zum Folgenden Bogumil 2018). Dabei wurden seit Mitte der neunziger Jahre mindestens 150 externe verwaltungswissenschaftliche Gutachten in Auftrag gegeben, dazu wurden ca. 15 Enquetekommissionen eingesetzt. Es dominieren politik- und verwaltungswissenschaftliche Stellungnahmen, diese sind aber auf wenige Personen beschränkt. Ein Grund für diese intensive Nutzung von Expertenwissen ist, dass die Reformmaßnahmen, vor allem Gebiets- und Funktionalreformen, häufig politisch sehr umstritten sind, und die Hinzuziehung von externem Wissen daher helfen soll, die Legitimation der Maßnahmen abzusichern.

In einer genaueren Untersuchung, in der danach gefragt wird, wie und wann welche Experten ausgewählt werden, ob es Einflussversuche seitens der Auftraggeber gibt und wie die Prozesse der Ergebnisverwendung verlaufen zeigt sich, dass sich die Logik wissenschaftlicher Expertise mit der Logik politischer Entscheidungsrationalität verkoppeln muss, damit wissenschaftliche Erkenntnisse überhaupt eine Chance haben, die Verwaltungspraxis zu verbessern.

„Dies erfordert die Bereitschaft von Wissenschaftlern, sich in die Entscheidungslogiken von Verwaltung und Politik einzudenken (und z.T. in dieser zu agieren), um überhaupt in den Wahrnehmungshoriziont der Praxis vorzudringen. Dann besteht eine Chance, wissenschaftliche Argumente in politische Prozesse einfließen zu lassen, wohlwissend, dass zumindest im Feld der Verwaltungspolitik sich im Zweifel die Machtlogik durchsetzt“ (Bogumil 2018, S. 157).

77 Natürlich spielen auch Policy-Probleme wie die „Flüchtlingskrise“, Arbeitsmarktprobleme oder der technische Wandel (Digitalisierung) eine wichtige Rolle. Diese Debatten verbinden sich dann häufig mit Verwaltungsreformdiskussionen. 
Wissenschaftliche Beratung hat aber dennoch erhebliche Einflussmöglichkeiten, allerdings werden diese Beratungschancen bisher eher unzureichend genutzt:

\begin{abstract}
„Zum einen gibt es - trotz der zunehmenden Tendenzen, Universitäten die Rolle einer third mission ${ }^{78}$ zuzuweisen - insbesondere in Teilen der Sozialwissenschaften immer noch Bedenken, dass Politik- oder Praxisberatung „unwissenschaftlich“ sei. Möglicherweise ist dies auch dadurch inspiriert, dass man Angst hat, in Machtprozesse involviert zu werden. Eine zweite Barriere könnte in der internen Logik des Wissenschaftssystems liegen, welches sich stärker an den jeweiligen disziplinären Communities orientiert. Damit ist es heute in vielen Fächern möglich, eine erfolgreiche wissenschaftliche Karriere mit wissenschaftlichen Arbeiten zu machen, die von nur wenigen „Peers" der eigenen Fach-Community rezipiert, aber für methodisch gut befunden werden, selbst wenn diese Forschung keinerlei gesellschaftliche Relevanz erkennen oder erwarten lässt" (Bogumil 2018, S. 175).
\end{abstract}

In bestimmten Bereichen gibt es allerdings auch Hinweise auf eine Renaissance "expertokratischer" oder technokratischer Positionen, also der Forderung, die Politik solle sich viel stärker als bisher an dem orientieren, was ihr von Experten gesagt wird. Der Wunsch nach einer objektivierten Politikgestaltung auf der Basis gesicherten Expertenwissens wird insbesondere unter dem Schlagwort einer evidenzbasierten Politik (evidence based policy making) vertreten (Blätte 2019, S.34; zusammenfassend Cairney 2016). Dieses Vertrauen in wissenschaftliche Daten und Modelle ist in Deutschland insbesondere in der Klimapolitik oder im Gesundheitswesen (Saretzki 2019) verbreitet, in der Bildungspolitik eher kontrovers (siehe die PISA-Studien der OECD). Allerdings zeigen sich auch hier die Grenzen dieses Vertrauens: während die Prognosen der Klimaforscher weitgehend unbesehen geglaubt werden, wird z. T. von der gleichen Klientel der überwältigende Konsens, was die Unwirksamkeit homöopathischer Medizin angeht, ignoriert (siehe zu den kulturellen Grundlagen der Risikowahrnehmung immer noch grundlegend Douglas/ Wildavsky 1983). Umgekehrt argumentieren gerade populistische Positionen mit prinzipiellem Misstrauen gegenüber Experten. Gerade in der Coronakrise hat sich gezeigt, wie stark die Politik auf die Expertise von Virologen angewiesen ist und auf diese zurückgegriffen hat. Man hat intensiv auf Experten gehört und mit diesen diskutiert, aber auch dort gab es Unsicherheiten und Dissens. Dabei ist die Politik weitgehend offen mit diesen Unsicherheiten umgegangen und hat immer wieder versucht, ihre Maßnahmen zu erklären.

78 Gemeint ist damit, die Hochschulen neben ihren beiden ersten „Missionen“, Forschung und Lehre, stärker auf ihre gesellschaftliche Verpflichtung zu orientieren und ihre gesellschaftliche Orientierung zu verstärken. 
Jenseits der Kontroversen, wie stark sich Politik auf Experten verlassen soll, gibt es einen weitgehenden Konsens über die steigende Bedeutung wissenschaftlicher Erkenntnisse in der Formulierung und Umsetzung von Politik. Wenn man unter Nutzung nicht nur die konkrete Anwendung wissenschaftlicher Ergebnisse bei einzelnen Entscheidungen versteht, sondern umfassender die Beeinflussung der Perzeption und Interpretation sozialer Phänomene, ihrer Ursachen und möglicher Veränderungen, spricht vieles dafür, dass wissenschaftliche Beratung erheblichen Einfluss hat. Von einigen Beobachtern (vgl. grundlegend Weiss 1977) wird seit langem die These vertreten, dass „wissenschaftliche Erkenntnis“, z. B. über die Ausgestaltung oder die Wirkung von Policies, nicht einfach von Verwaltung und Politik übernommen wird, indem z. B. Wissenschaftler befragt oder die Thesen oder Ergebnisse von Untersuchungen gelesen und dann einfach umgesetzt werden (auch und gerade nicht bei bestellten Gutachten). Die zentrale Bedeutung der Wissenschaft für die Praxis liegt vielmehr darin, dass sie Daten, Konzepte und Denkschemata bereitstellt, mit denen die Realität neu geordnet und interpretiert wird. Praktiker übernehmen in der Regel keine fertigen Lösungen oder abstrakten Theorien, sondern werden durch Begriffe, Konzepte und Sichtweisen der Wissenschaft beeinflusst. In diesem "diffuse process of enlightenment" (Carol Weiss), also in einem langfristigen und wenig strukturierten Prozess der "Aufklärung“, liegt daher die zentrale längerfristige Bedeutung und Wirkung von Wissenschaft.

Dazu gehört auch die Erkenntnis, dass der Zugang zu wissenschaftlichem Wissen längst kein Privileg von Regierungen mehr ist (Weingart 2019, S. 68). Weil Wissen eine strategische Bedeutung im politischen Machtkampf haben kann, versuchen alle Parteien, Verbände, Unternehmen und NGOs sich das aus ihrer Perspektive relevante Wissen zu beschaffen und zu kontrollieren. Wissen war schon immer Munition in politischen Auseinandersetzungen. Dies stimmt auch mit der Beobachtung überein, dass sich ein großer und zunehmend wichtiger Teil der Politikberatung heute nicht mehr ausschließlich an Politik und Verwaltung richtet, sondern die öffentliche Diskussion und Konsensbildung beeinflussen will, z. B. durch öffentlichkeitswirksame Gutachten und Präsentationen. Ein wichtiger Teil der Politikberatung ist daher heutzutage Gesellschaftsberatung (Leggewie 2007, Bertelsmann Stiftung 2011), aber auch diese Beratung wirkt natürlich in erster Linie, sozusagen „über Bande“, durch den professionellen Verwaltungsapparat auf Politik und Regierung. 